\title{
Iscs
}

IRANIAN SOCIETY OF CARDIAC SURGEONS

\section{Supplement Issue}

Volume 10

\author{
Abstract Presentations \\ 7th Iranian Joint Cardiovascular Congress \\ 27 Feb.- 1 March 2019
}

Rajaie Conference Center Tehran/Iran 



\section{JOURNAL INFORMATION}

\section{- AIM AND SCOPE}

The official journal of Iranian Society of Cardiac Surgeons (ISCS) was founded in march 2007 entitled Iranian Journal of Cardiac Surgeons and was a national journal with the aim of scientific improvement and creation of a connection between cardiac surgeons. From fall 2016, this journal is going to be republished with a new international standard format under the name of Multidisciplinary Cardiovascular Annals (MCA). The main objective of this journal is to cover multidisciplinary aspects focusing on cardiovascular diseases and serve as a global forum for scientists, researchers, physicians, surgeons and other health professionals to find current advances in the areas of Cardiology and Cardiovascular Diseases.

\section{- CONTENT COVERAGE}

Multidisciplinary Cardiovascular Annals welcomes submissions in various article types with hopes to create a compilation of the newest researches and findings in the area of cardiovascular diseases including Original Articles, Research Articles, Review Articles, Short Reports, Case Reports, Perspectives (Editorials) and supports scientific innovations and advancements in all related aspects of cardiovascular diseases by increasing access to peer-reviewed scientific literature.

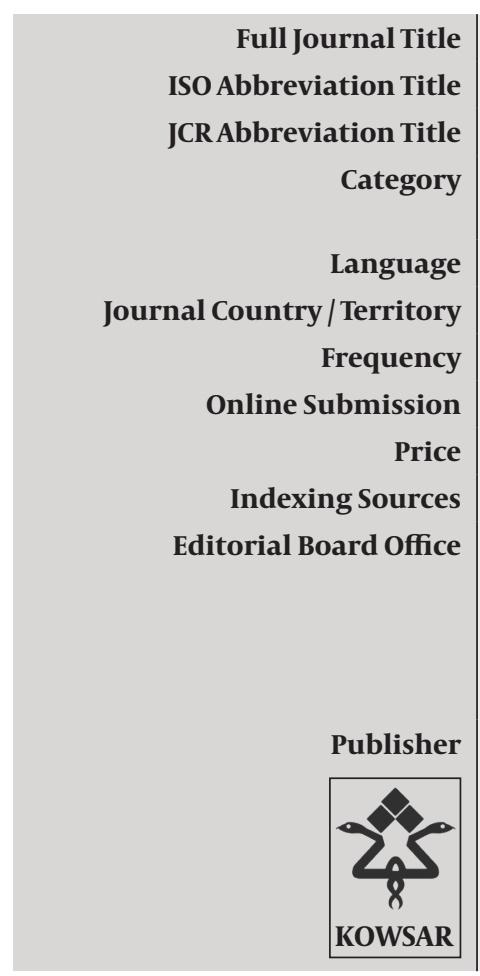

Multidisciplinary Cardiovascular Annals

Multidiscip. Cardiovasc. Ann.

MULTIDISCIP CARDIOVASC ANN

Cardiac Surgery, Cardiology, Pediatric Cardiology, Vascular Diseases, Cardiac Anesthesia and Cardiac Imaging

English

IR Iran

Quarterly

www.multicardia.com

Online: Open Access; Hardcopy: Free of charge for ISCS members

Open J Gate, Index Copernicus, Google Scholar, MagIran

Postal Address:

Unit 2, No. 4, Nikray Alley, Kazeroon St, Mirdamad Blvd, IR Iran; Tel: +98-2126401340;

Fax:+98-2122279743

- Electronic Address:

Website:www.multicardia.com | E-mail: editor@multicardia.com

Kowsar Medical Institute

- Postal Address:

- Central Office: Huyn van Rodenbroeckstraat 61, 6413 AN Heerlen, The Netherlands, Cell: +31614212157

\& +31615314198 | Fax: +31452051123 .

> Electronic Address:

Website:www.kowsarpub.com |E-mail:info@kowsarpub.com 



\section{CONTENTS}

Volume 10, Supplement Issue

- Introduction.

\section{Oral Presentations}

- Trends of major cardiovascular risk factors in Iranian population during 13 years Isfahan Cohort Study. .........................

- Exposure to Occupational Air Pollution and Vascular Endothelial Dysfunction in Workers of the Esfahan Steel Industry Iran.........4

- Study of secretomes from autologous and allogenic adipose bone marrow umbilical cord blood-derived mesenchymal stem cells on in vitro model of ischemia reperfusion using induced pluripotent stem cell-derived cardiomyocytes. ...

- Proprotein Convertase Subtilisin-Kexin Type 9 PCSK9 Inhibitors for goal-inhibiting statin intolerant and goal-inhibiting statin resistant patients a systematic review......

- Major cardiovascular risk factors patterns during 13 years follow-up among Iranian adults Isfahan Cohort Study..................5

- The association of serum HbA1C level and heart failure severity in systolic heart failure patients with diabetes...................5

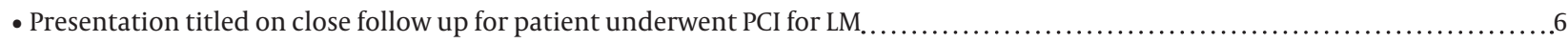

- Prosthetic Valve thrombosis Replacement or Preserving the Valve Single Center Experience...................................6

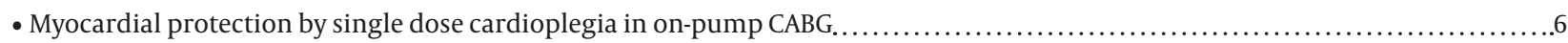

- No need to stop taking aspirin and clopidogrel before open-heart surgery ...................................................

- Coronary Artery Fistulas A case series of surgical results and clinical characteristics in Iranian population.......................7

- An Investigation of Changes in the Quality of Life of Patients Undergoing Minimally Invasive Mitral Valve Surgery and Surgical Ablation for Atrial Fibrillation Using Ex-Maze III Procedure.

- Mid-term Results of the First Series of Minimally Invasive Cardiac Surgeries in Rajaei Cardiovascular Medical and Research Center..8

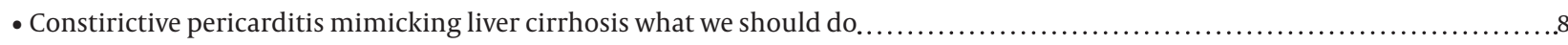

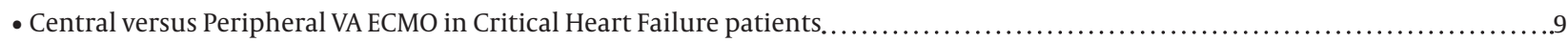

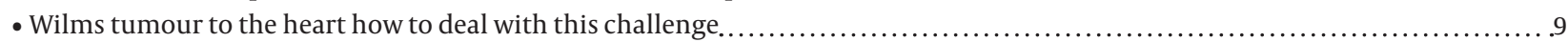

- Prevalence of Anemia In Patients Undergoing Cardiac Surgery in Rajaie Heart Center And Need for Transfusion of Blood During Operation Regarding to Hemoglobin Level.

- Comparison of the malposition between midpoint and Lateral point approaches for subclavian vein catheter placement in patients undergoing cardiac surgery.

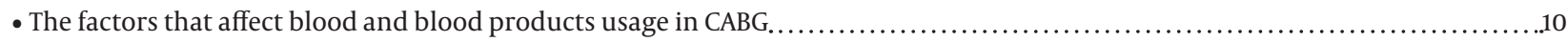

- The Effect of Dexmedetomidine on Hemodynamic Responses during Tracheal Extubation ....................................10

- An assessment of the renal function with pulsatile perfusion during the proximal graft by using cardiac contraction in CABG surgery....11

- The relationship between fluid balance and the incidence of post-operative acute kidney injury during and 24 hours after CABG surgery.

- Comparison of modified tight control versus conventional glucose control methods among diabetic patients undergoing cardiac surgery.

- Non-surgical treatment of right ventricular muscle band in a child right ventricular outlet tract stenting

- The effect of Atorvastatin on mortality and complications of pulmonary embolism short and long-term follow-up.................12

- The predictive role of Insulin-like growth factor-1 and Pregnancy-associated plasma protein A biomarkers in acute coronary

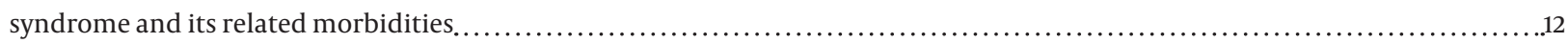

- Nonpharmacologic treatments alone are enough to prevent neurally mediated syncope A 3 year follow up study................12

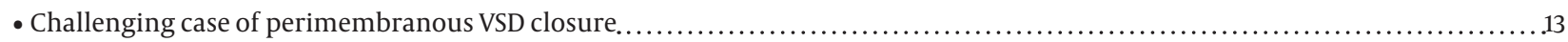

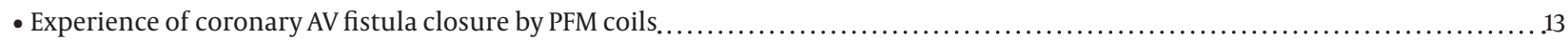

- Heart function and Remodeling after successful stenting of Coarctation of Aorta in children...................................13

- Rehabilitation of the Pulmonary Artery in Tetralogy of Fallot with Unilateral Absent Pulmonary Artery..........................14

- Evaluation of etiology and time between tetralogy of Fallot total correction and pulmonary valve replacement through 5 years ago in Tabriz Shahid Madani hospital.

- Relation of preoperative diastolic pulmonary gradient and pulmonary compliance with early post-operative mortality after cardiac 


\section{CONTENTS}

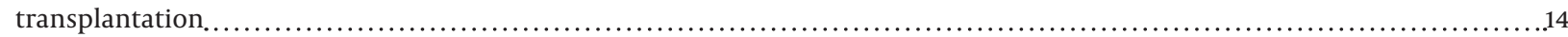

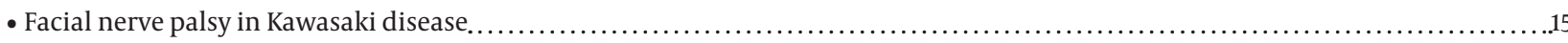

- Prevalence and risk Factors of Attention Deficit hyperactivity disorder After Artrial Switch Operation in Neonatal Period............15

- A Large Venovenous Collateral from Renal Vein as a Cause of Desaturation in Fontan Circulation.................................15

- The effect of protocol weaning from ventilator on the duration of mechanical ventilation in patients with acute kidney injury after

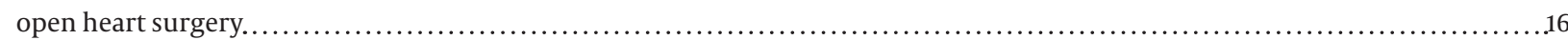

- The effect of using standard checklist on nursing handover status in cardiac care units of Afshar hospital in Yazd ..................16

- The Relationship between Health Literacy and Adherence to Treatment in Cardiac Patients.....................................16

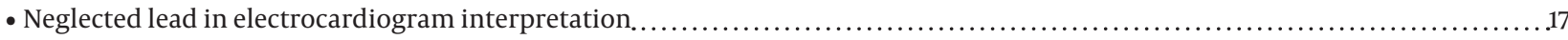

- Assessing serum fibrinogen and fibrin degradation products FDP changes in hypothermia and normothermia in patients undergoing

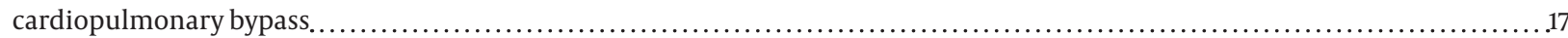

- Hyperthermic Intra-Operative Chemotherapy for Management of Peritoneal Carcinomatosis with a cardiopulmonary bypass

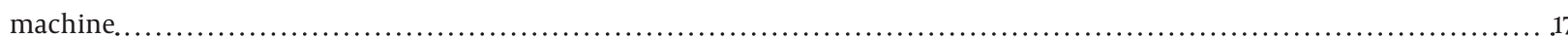

- Recommendations for Standards of Monitoring and Alarms during Cardiopulmonary Bypass .................................18

- Serum levels of IL-6 IL-10 and TNF- $\alpha$ renal function biochemical parameters and patients' outcomes in pediatric cardiopulmonary bypass surgery.

- The Influence Of Mean Arterial Blood Pressure During Cardiopulmonary Bypass On Post Acute Kidney Injury AKI In Hypertensive Patients

- Effect of Adenosine Fast Cardioplegic Arrest Induced on Troponin Levels After On-pump bypass surgery .

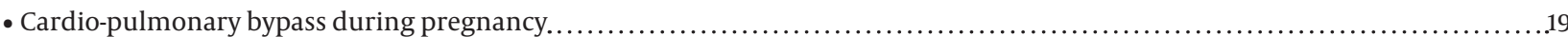

- The effect of Delnido and Custodiol cardioplegia on peri-operation clinical outcomes in patients with Tetralogy of Fallot who

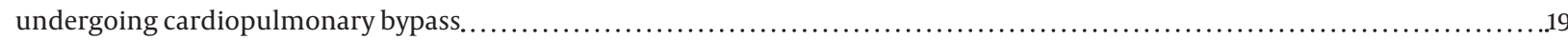

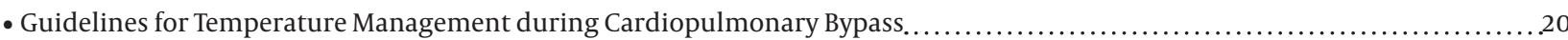

- Clinical and laboratory results of adding Albumin Versus Fresh Frozen Plasma in Priming Solution of CPB in Pediatric Patients....20

- The survival rate and complications after congenital heart surgery in children under membrane oxygenation machine outside the body ECMO

- Zero-Balance Ultrafiltration of the Priming Blood Modifies the Priming Components and Improves the Clinical Outcome in Infants Undergoing Cardiopulmonary Bypass A Randomized Controlled Trial....

- Correlation of rewarming time with arterial blood gas parameters in patient undergoing Coronary Artery Bypass Graft surgery . . .21

- The effect of different phases of menstrual cycle on respiratory indices in primary dysmenorrhea women. . . . . . . . . . . . . . ....22

- A Review of Telerehabilitation Methods for Monitoring Cardiovascular and Pulmonary Patients outside the Rehabilitation Centers. . . 22

- Effects of resistance training on cardiovascular parameters and glycemic control in patients with type 2 diabetes...............22

- Reaction Time and Anticipatory Skill in Patients Undergoing Percutaneous Coronary Intervention Compared with Normal Control A

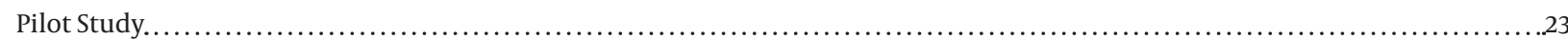

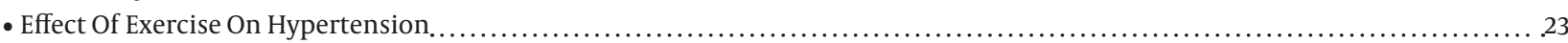

- Effect of Cardiac Rehabilitation on Lipid Profiles in Patients with Cardiovascular Disease A Systematic Review.....................24

- Effect of aerobic and resistance exercises on cardiovascular fitness functional ability and muscle strength in children with

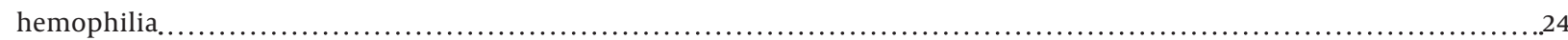

- Effect of Cognitive Behavior Therapy on cardiac rehabilitation Review article . ..........................................24

- The Investigation of Barriers to Participation in Cardiac Rehabilitation and How to Overcome ................................25

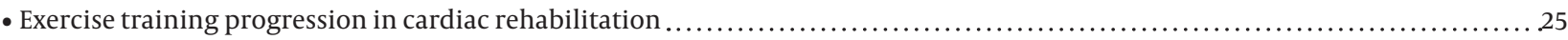

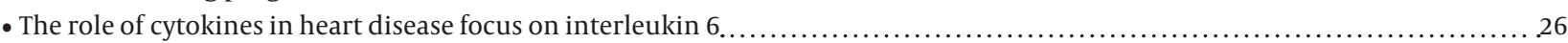

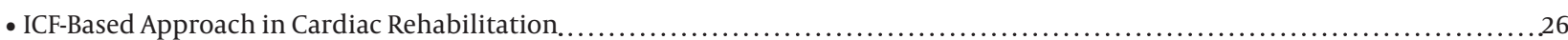

- Implementation of An ECG Holter Monitor Recorder and Analyzer Software, and its Clinical Trial ..............................26

- An Ultra-Low-Power IC for Implantable Cardiac Pacemakers and/or Ambulatory ECG Recorders. ..............................26

- Burden of Structural Heart Diseases in School-Aged Population: Protocol of SHED LIGHT Study (Structural HEart Diseases in pupiLs by

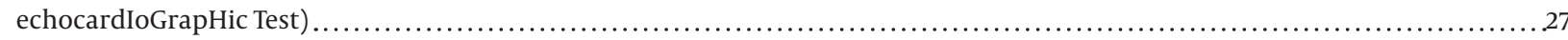

- How is it possible to increase the safety in cardiac surgery "Twenty years experiences" ...................................27

- Efficacy of Dexmedetomidine in Coronary Artery Bypass Graft Surgery under Cardiopulmonary Bypass. .......................28

- Effects of resistance training on cardiovascular parameters and glycemic control in patients with type 2 diabetes................28

- Major cardiovascular risk factors patterns during 13 years follow-up among Iranian adults Isfahan Cohort Study..................28

- Comparative study of cardiac muscle damage markers and clinical outcomes in patients undergoing pulmonary valvular surgery using a cardiopulmonary bypass device using two Beating Heart pump and non-beating heart pump methods ................29

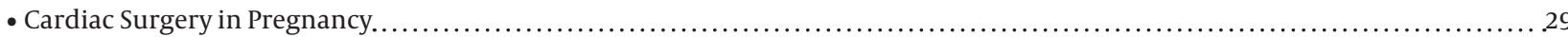




\section{Poster}

- Hypertension prevalence and its association with anthropometric indices among Iranian adults. ....

- Amazing in hospital primary percutaneous coronary intervention results in Farshchian Heart Center............................3. 32

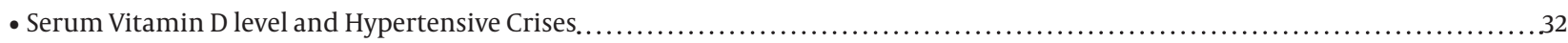

- The role of genetic counselling and familial screening in the management of Hypertrophic Cardiomyopathy....................33

- The relationship between socioeconomic level and cardiovascular risk factors in patients with severe coronary angiography .......33

- Do patients with coronary artery bypass grafts using radial artery need special nursing care ..............................33

- Comparison of mean 2nd and 3rd blood pressure measurements with other reading frequencies among Iranian adults...........34

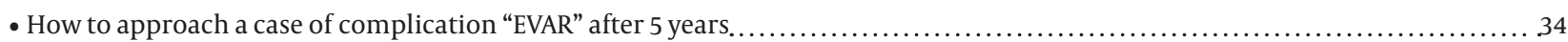

- Angiographic evaluation of graft patency in patients with prior CABG refered to north of Iran between 2013-2017................34

- Title A rare complication of PCI Percutaneous Coronary Intervention that leaded the patient to CABG Coronary Artery Bypass

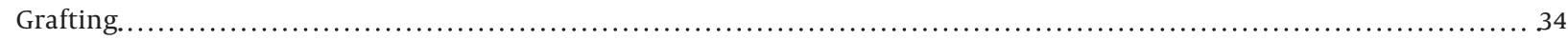

- Novel distal accesses in the hand for percutaneous coronary angiography and intervention...................................35

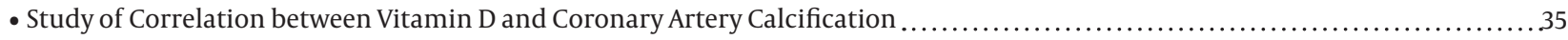

- Impact of Procaine Hydrochloride Versus Lidocaine in Cardioplegic Solution on Reperfusion Arrhythmia During Coronary Artery

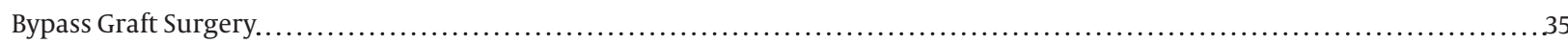

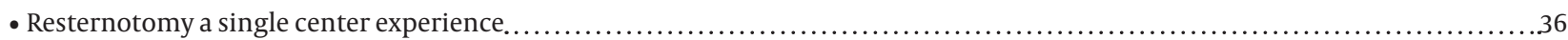

- Exposure of electromagnetic field emitted by mobile phone on cardiac rhythm disorders of patients with Aortic valve replacement. 36

- The effect of topical use heparinized own blood on post operation blood loss in patient with open heart surgery a randomized

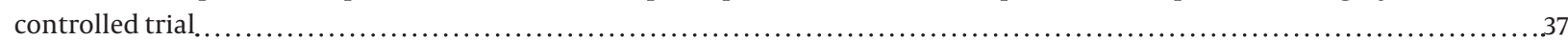

- Some factors associated with hospital mortality in adult patients undergoing open-heart surgery ............................37

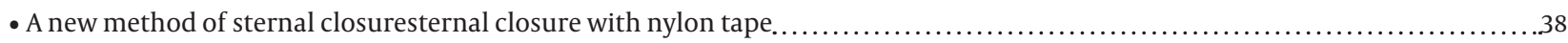

- Prevention of Post-Operative Arrhythmias in Coronary Artery Bypass Graft OperationsUse of Magnesium Sulfate.................. 38

- Optimizing venous drainage using a modified roller pump without oxygenator in surgical management of right heart diseases. . 38

- What do you know about your child's disease A Rough Assessment of Parents' knowledge about Their Child's Congenital Heart Disease.

- A rare coronary artery variant super-dominant Left Anterior Descending coronary artery.......................................39

- Speckle Tracking Echocardiography Findings in Healthy Individuals with Fragmented QRS .................................

- Frequency of Reoperation due to Bleeding after Adult Cardiac Surgery at Rajaie Heart Center..................................40

- Short-term Outcome of Patients with Infective Endocarditis A Single-center Prospective Study. .............................40

- Effect of Remote Ischemic Preconditioning on ischemic biomarkers in Coronary Artery Bypass Graft...........................41

- Comparison of invasive and noninvasive blood pressure measurement in patient's candidate to coronary artery bypass graft under

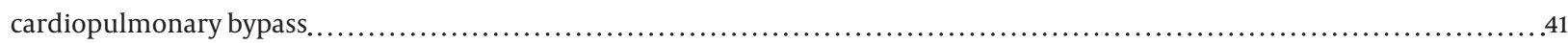

- Evaluation of the tracheal tube cuff pressure changes during cardiac operations under cardiopulmonary bypass................41

- Comparison of acute normovolemic hemodilution effect on the amount of bleeding in the first 48 hours after coronary artery

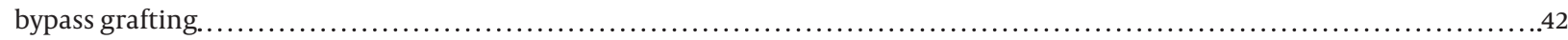

- New cardiology clinic conformation new horizon of research and patient care ...........................................42

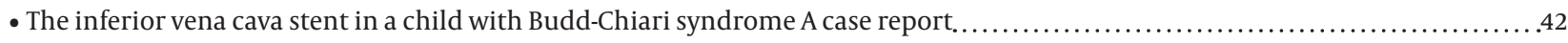

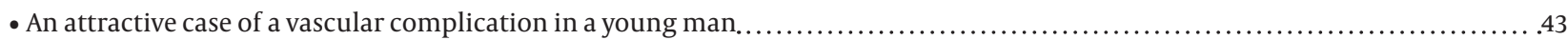

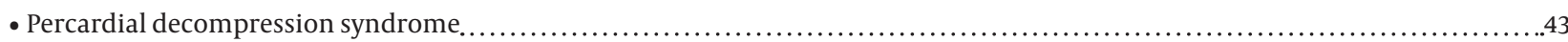

- The role of vitamin D on the left ventricle ejection fraction in thalassemia major patients with cardiac hemosiderosis in children's

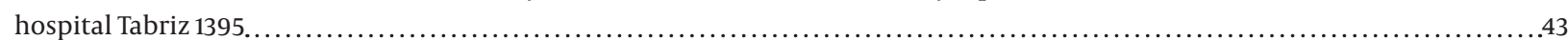

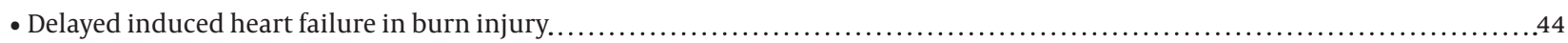

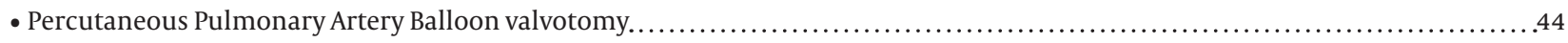

- Aspirin response monitoring by impedance aggregometry in children with systemic-to-pulmonary shunts or PDA stent..........44

- Enteral Nutritional Support by Nurses and Related Factors in Intensive Care Patients. ....................................45

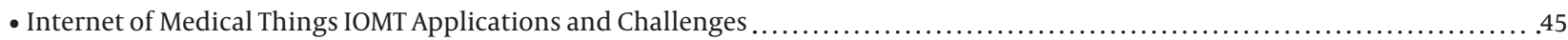

- Association between fear of falling and functional dependency in heart failure patients in 2018 Tehran-Iran .....................46

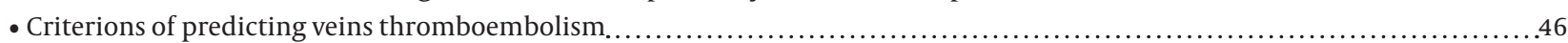

- Processes underlying concordance to treatment regimen A grounded theory study ........................................

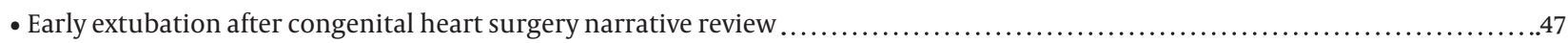

- An evaluation of the effectiveness of social media-based training on satisfaction and anxiety of families of patients who are under Coronary artery bypass surgery at Intensive Care Unit ICU. 
-Validity and Reliability of the professional communication skills of nurses Questionnaire ..............................47

- Evaluation of the Critical thinking in the nurses who work at the Intensive Care Units in hospitals of Universities of Medical Sciences . . .47

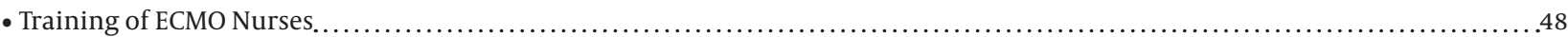

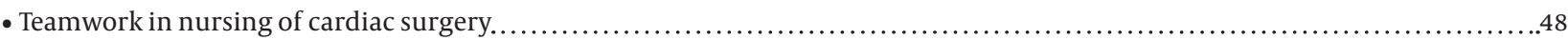

- Preparation and spiritual Support of Patients and their families through the heart Transplant Process .........................48

- Reducing the anxiety in parents of children with congenital heart anomaly and its effect on the treatment process..............48

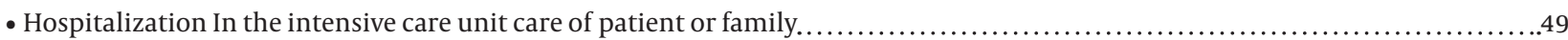

-Wearable cardioverter-defibrillator for primary prevention of post-myocardial infarction sudden cardiac death.................49

- Impact of Door to ECG on patients refer to the emergency department with chest pain....................................49

- Hospital Facilities at Home for Heart Failure Patients A cost-effectiveness study...........................................50

- Assessment The Role of Nurses in Promoting Lifestyle in Patients with Heart Failure A Review Study............................50

- Assessment of Factors Impacting the Quality of Life in Patients with Hypertension Systematic Review Literature ...................50

- Guide line by JNC-8 in patients who referred to health centers survey of accumulation of hypertension treatment with last related to

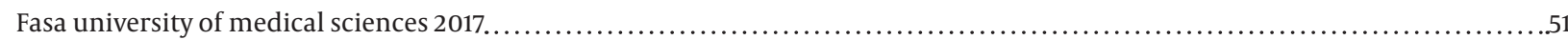

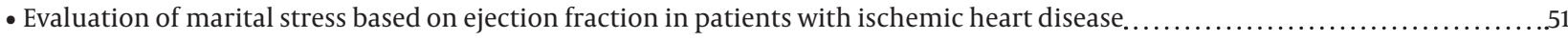

- Catecholamines and broken heart syndrome Are catecholamines guilty With a look at nursing care ............................51

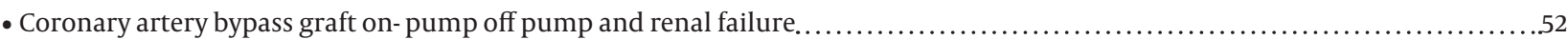

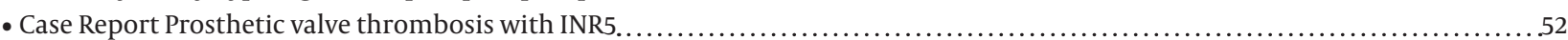

- Association Body mass index waist circumference and waist hip ratio with cardiovascular risk .............................52

- Reasons for the termination of pregnancy in hospital women with heartdisease in Shahid Madani Heart center in Tabriz..........53

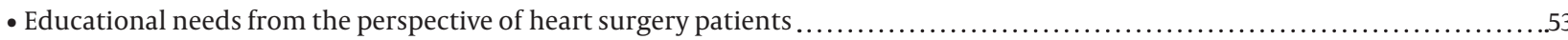

- Congenital heart disease abnormalities in East west of Azerbaijan in 2018 A new approach to improve the pregnant mother with

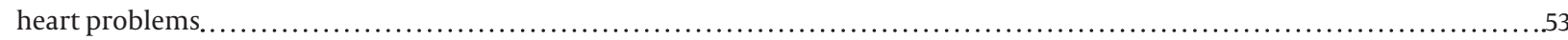

- Correlation between arterial and venous blood gases in patients with mechanical ventilation after intracranial surgery............54

- The role of Vitamin C in the Prevention of Post operative Complication after Cardiac Surgery ................................54

- Effect of Cardiopulmonary Bypass Machine on Serum Vitamin D3 Level in Pediatrics Open Heart Surgery review..................54

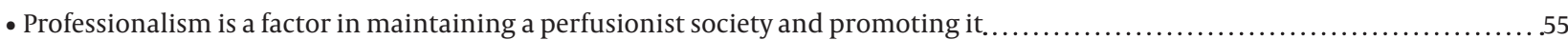

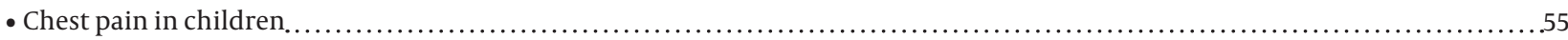

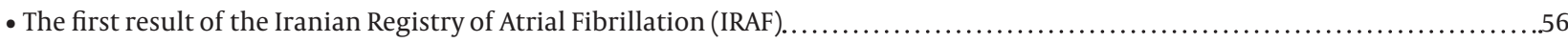

- Trend of salt intake measured by 24-hour urine collection samples among Iranian adults, 1998-2013: The Isfahan Salt Study ........56

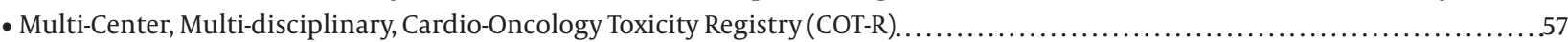

- Comparison of 2 days oral Acetaminophen and oral Ibuprofen on the closure of patent ductus arteriosus PDA in premature neonates ...58

- Evaluation the effect of premedication with intravenous paracetamol on hemodynamic changes during laryngoscopy in children

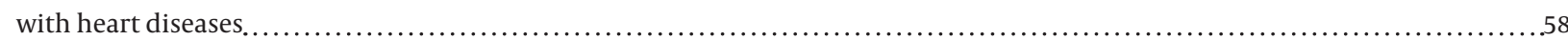

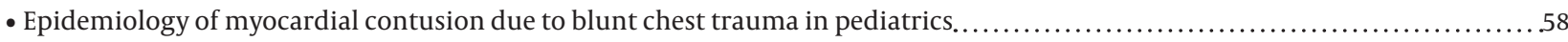

- The introduction of four cases of severe infantile dilated cardiomyopathy that were improved with medium-chain triglyceride ...59

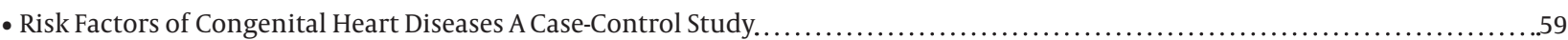

- The commencement of congenital heart disease registry in Iran-Isfahan Methodology and Design...........................59

- Oxygen therapy in patients with acute myocardial infraction the problem that is not yet solved ...............................60

- A case series of pulmonary hypertension in children under six months old with Atrial Septal defect..........................60

- Prevalence of Cardiac Involvement in Patients with Rheumatic Fever at Tabriz pediatric Hospital from 2006-2016 ...............60

- Evaluation of pulmonary complications after open cardiac surgery in children with Congenital anomalies ....................61

- The Frequency rate of early postoperative complete heart block in pediatric patients submitted to open-heart surgery for congenital

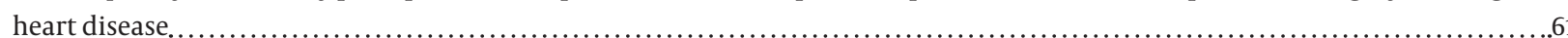

- Prevalence of congenital heart disease in pregestational and gestationaldiabetes melitus ...................................61

- Determining the prevalence of early and late complications following the Truncus Arteriosus reconstructive surgery at Shahid Rajaie

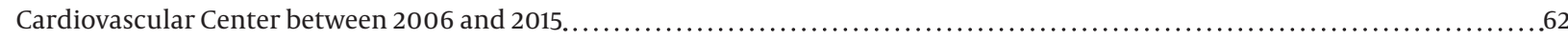

- Right atrium thrombosis as a late onset complication of surgical ASD closure in a 6 -year-old girl ............................6. 62

- Diagnostic and therapeutic pediatric cardiac catheterization A large single-center experience ..............................62

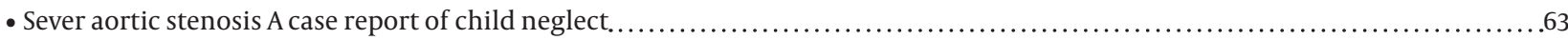

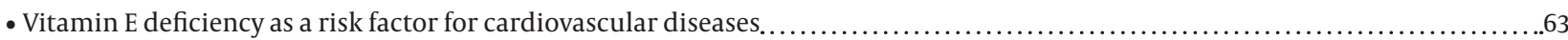

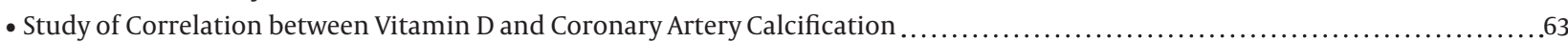

- The relationship between QT interval and Severe pulmonary hypertension in patients with congenital heart disease ..............63

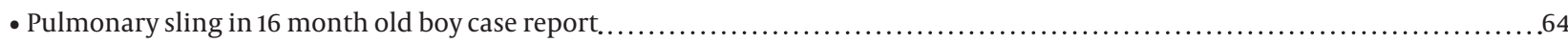

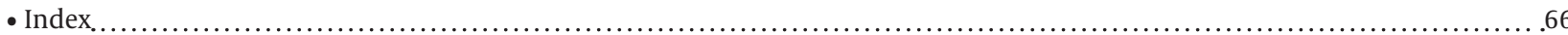




\title{
In The Name of God
}

Cardiovascular diseases remain as a leading cause of death, despite a great number of clinical researches, significant improvements in diagnostic tools, prevention strategies, medical managements, and interventional or surgical techniques. Annual Iranian joint cardiovascular congress (IJCC) is a wonderful opportunity for sharing valuable experiences of the international and national experts in all cardiovascular disease fields. In the 7th IJCC that will be held in Rajaie Conference Center in Tehran, twelve national scientific societies will have different joint meetings in addition to specific sessions. At the first day of this event, an interesting valve repair workshop will be moderated as a live in box session by a well-known cardiac surgeon, Professor Gebrine El- Khouri from Belgium. General issues like Heart failure, diabetes and heart disease, debates on myocardial revascularization, epidemiologic aspects of heart disease and horizon in cardiovascular disease will be discussed in the main hall. For the first time, two new topics of "highlights of cardiovascular researches in Iran" and "the role of technology in the practice and promotion of cardiovascular disease" have been added to the main hall programs. Physicians, cardiac nurses and physiotherapists, and also perfusionists have submitted 302 abstracts. After online review process, 74 abstracts have been selected for oral and 104 for digital poster presentations.

I would like to invite you to participate in this major national cardiovascular event and meet the experts from different countries like France, Germany UK, Switzerland, Belgium, Italy, Turkey, Oman, Qatar, UAE and also our beautiful country Iran.

\author{
Alireza Alizadeh Ghavidel \\ Editor in Chief
}





\section{Oral Presentations}




\section{- Trends of major cardiovascular risk factors in Iranian population during 13 years Isfahan Cohort Study}

\author{
Sadeghi M. ${ }^{1}$, Hesmat K. ${ }^{2}$, Roohafza H. ${ }^{3}$, \\ Sarrafzadegan N. ${ }^{3}$
}

${ }^{1}$ Cardiovascular Research Institute Isfahan University Of Medical Sciences, Cardiac Rehabilitation Research Center, Isfahan, Iran

${ }^{2}$ Cardiovascular Research Institute Isfahan University Of Medical Sciences, Heart Failure Research Center, Isfahan, Iran

${ }^{3}$ Cardiovascular Research Institute Isfahan University Of Medical Sciences, Isfahan Cardiovascular Research Center, Isfahan, Iran

\section{Abstract}

Backgrounds: Trends of major cardiovascular risk factors are less investigated in Middle-East countries. In this study we aimed assessing these factors among Iranian individuals by data of Isfahan Cohort study(ICS).

Material and method: Longitudinal population based study was performed on 6504 participants aged at least 35 years for 12 years initiated in 2001 till 2013in three phases. Cardiovascular risk factors including systolic blood pressure(SBP), diastolic blood pressure(DBP), waist circumference(WC), waist to hip ratio(WHR), body mass index(BMI), fasting blood sugar(FBS) and lipid indices were measured for each subject in 2001, 2007 and 2013. Data entered in spss v22 analyzed by T-test and Chi-square. Result: Mean value of SBP, DBP, BMI and FBS increased significantly in both genders during follow-up(SBP: 119.3 \pm 18.7 to128.4 \pm 17.5 $\mathrm{mmHg}$, P-value<0.001, DBP: $76.7 \pm 11.8$ to $2.8 \pm 12.5 \mathrm{mmHg}$, Pvalue $<0.001$, BMI: $27.4 \pm 4.4$ to28 $\pm 4.6 \mathrm{~kg} / \mathrm{m} 2$, P-value $<0.001$, FBS: $86.7 \pm 29.6$ to106.5 $\pm 38 \mathrm{mg} / \mathrm{dl}$, P-value $<0.001$ ). Lipid profiles including total cholesterol(TC), triglyceride(TG), low-density lipoprotein cholesterol(LDL-C) and TG/ high-density lipoprotein cholesterol(HDL-C) showed a favorable downward patterns in population(TC: $219.5 \pm 51.3$ to200.1 $\pm 40.9 \mathrm{mg} / \mathrm{dl}$, P-value $<0.001$, TG: $200.2 \pm 110.3$ to155.1 $\pm 85 \mathrm{mg} / \mathrm{dl}$, P-value $<0.001$, LDL-C: $132.6 \pm 42.4$ to111.7 $\pm 27.8 \mathrm{mg} / \mathrm{dl}$, P-value $<0.001, \mathrm{TG} / \mathrm{HDL}-\mathrm{C}: 4.5 \pm 2.8$ to3.9 \pm 2.8 , P-value $<0.001$ ) with an exception of HDL-C showing less desirable result. In spite of controversial results in terms of WC and WHR alteration specified by gender, total population changing of those variables were not significant.

Conclusion: Although favorable patterns were observed in terms of most lipid indices, other risk factors showed an inverse or insignificant trend. Several health promoting strategies required in order to improve lifestyle in a way that to those raising cardiovascular risk factors trends would be declined.

\section{- Exposure to Occupational Air Pollution and Vascular Endothelial Dysfunction in Workers of the Esfahan Steel Industry Iran}

\section{Sadeghi M. ${ }^{1}$, Golshahi J. ${ }^{2}$, Roohafza H. ${ }^{2}$, Sadeghifar M. ${ }^{2}$}

${ }^{1}$ Cardiovascular Research Institute Isfahan University Of Medical Sciences, Cardiac Rehabilitation Research Center, Isfahan, Iran

${ }^{2}$ Cardiovascular Research Institute Isfahan University Of Medical Sciences, Isfahan Cardiovascular Research Center, Isfahan, Iran

\section{Abstract}

Backgrounds: Air pollution is a risk factor for cardiovascular diseases, but the mechanisms are not clear yet. We investigated occupational air pollution exposure and endothelial function among workers of the steel industry.

Material and method: Workers of the coke-making part of the Esfahan Steel Company and workers in the administrative parts with no known history of cardiovascular risks were studied. Data of age, body mass index, duration of employment, blood pressure, fasting blood sugar, and lipid profile were gathered. Flow-mediated dilation (FMD) was measured to evaluate endothelial function.

Result: Baseline brachial artery diameter was greater (mean difference $[95 \% \mathrm{CI}]=0.068 \mathrm{~mm}[0.008$ to 0.128$]$ ), but the FMD was lower (mean difference $[95 \% \mathrm{CI}]=-0.908 \%[-1.740$ to -0.075$]$ ) in the coke-making group compared with controls. Controlling for possible confounders, working in the coke-making part of the industry was associated with lower FMD $(\mathrm{F}=3.954, \mathrm{P}=0.049)$.

Conclusion: These results show that occupational air pollution exposure in workers of the steel industry is associated with impaired endothelium-dependent vasodilation.

\section{Study of secretomes from} autologous and allogenic adipose bone marrow umbilical cord blood-derived mesenchymal stem cells on in vitro model of ischemia reperfusion using induced pluripotent stem cell-derived cardiomyocytes

\author{
Moslem F. ${ }^{1}$, Hosseini M. ${ }^{1}$, Halvaei M. ${ }^{1}$, Pahlavan S. ${ }^{1}$ \\ ${ }^{1}$ Royan Institute, Stem Cell Biology And Technology, Tehran, Iran
}

\begin{abstract}
Backgrounds: Cardiovascular diseases are among most prevalent health problems all over the world as well as Iran. Particularly, myocardial infarction causes $30 \%$ of death rate in the world, while 40 \% in Iran. Moreover, ischemic heart conditions can progress to heart failure. Chronic heart failure may result in serious conditions, making heart transplantation the only remaining clinical solution. However, there is limited number of organ donors which complicates this therapeutic solution. Last 2 decades, cell therapy was introduced as a promising approach to tackle these situations. Mesenchymal stem cells (MSCs) have been widely used in regenerative medicine due to their pluripotent characteristics and paracrine effects. In this study, we aimed to compare the cardiac repair effects of MSCs' secretome derived from three sources of adipose, bone marrow and umbilical cord in an in vitro model of hypoxia in human induced pluripotent stem cells (hiPSC)-derived cardiomyocytes.

Material and method: Hypoxia was induced in hiPSC-derived cardiomyocytes and neonatal mouse cardiomyocytes using 1 $\mathrm{mM} \mathrm{H} 2 \mathrm{O} 2$ for 4 hours. The hypoxia induced cardiomyocytes were then subjected to indirect co-culture with heat-shock treated MSCs in order to receive their secretome.

Result: Hypoxia induction was confirmed by translocation of HIF-1 $\alpha$ into nucleus as visualized by immunostaining of cells pre and posthypoxia. Electrophysiological evaluation of cardiomyocytes as well as their Ca2+ imaging post-MSC secretome reperfu-
\end{abstract}


sion showed that umbilical cord (Wharton jelly) MSC could better repair cardiomyocytes to their prehypoxia condition. Conclusion: In conclusion, Wharton jelly MSC might be the best mesenchymal stem cell source for cardiac cell therapy.

\section{Proprotein Convertase Subtilisin-} Kexin Type 9 PCSK9 Inhibitors for goalinhibiting statin intolerant and goalinhibiting statin resistant patients a systematic review

\author{
Bakhshandeh H. ${ }^{1}$, Fouladi Vanda H. ${ }^{2}$, Amin A. ${ }^{3}$ \\ ${ }^{1}$ Rajaie Cardiovascular Medical And Research Center Iran University Of Medical Sci- \\ ences Tehran Iran, Epidemiology, Tehran, Iran \\ ${ }^{2}$ Rajaie Cardiovascular Medical And Research Center Iran University Of Medical Sci- \\ ences Tehran Iran, Cardiology, Tehran, Iran \\ ${ }^{3}$ Rajaie Cardiovascular Medical And Research Center Iran University Of Medical Sci- \\ ences Tehran Iran, Heart Failure, Tehran, Iran
}

\section{Abstract}

Backgrounds: Cardiovascular diseases are the leading cause of mortality and morbidity worldwide. Evidenced based scientific reports, show that lipid lowering strategies (e.g. medical, surgical or drug based) decrease cardiovascular morbidity and early mortality. Nowadays, statins are the most effective and favorable lipid lowering agents which can reach more than $50 \%$ reduction in serum LDL-C, based on usage dose, and also have more effects other than lowering of serum lipid levels. The patients who cannot tolerate recommended doses of statins or are non-responsive to such doses, experience more cardiovascular events. Conventional non-statin agents (e.g. ezetimibe, fenofibrates and etc.) as alternative therapy, for goal-inhibiting lipid lowering strategies are not as potent as statins are, in specific groups who are resistant or intolerant to statins, and are not as effective as statins are to prevent cardiovascular morbidity and mortality. So there is an increasing interest to find substitution agents such as PCSK9 inhibitors in this challenging groups, which can be potent and also safe.

Material and method: In this registered systematic review (PROSPERO code: CRD42018085959) of RCTs, we searched in electronic databases and found 32 eligible articles (among 1170 articles) based on prior defined inclusion/exclusion criteria. Quality assessment and then data extraction were performed. Population was defined as the patients who are goal-inhibiting statin intolerant or resistant.

Result: PCSK9 inhibitors can significantly reduce serum LDL-C in short- ( $<24$ weeks) to mid-term follow up (up to 26 months) and significant reduction of cardiovascular events in mid-term follow up was seen with acceptable side effects.

Conclusion: as result

\section{Major cardiovascular risk factors} patterns during 13 years follow-up among Iranian adults Isfahan Cohort Study

Heshmatghahdarijani ${ }^{1}$, Sadeghi M. ${ }^{2}$, Sarrafzadegan N. ${ }^{3}$, Dianatkhah M. ${ }^{4}$, Vakhshoori M. ${ }^{5}$, Roohafza H. ${ }^{6}$
${ }^{1}$ Isfahan University Of Medical Sciences Isfahan Iran, Hypertension Research Center Cardiovascular Research Institute, Isfahan, Iran

${ }^{2}$ Isfahan University Of Medical Sciences Isfahan Iran, Cardiac Rehabilitation Research Center Cardiovascular Research Institute, Isfahan, Iran

${ }^{3}$ Isfahan University Of Medical Sciences Isfahan Iran, Isfahan Cardiovascular Research Center Cardiovascular Research Institute, Isfahan, Iran

${ }^{4}$ Isfahan University Of Medical Sciences Isfahan Iran, Heart Failure Research Center Cardiovascular Research Institute, Isfahan, Iran

${ }^{5}$ Isfahan University Of Medical Sciences Isfahan Iran, Cardiac Rehabilitation Center Isfahan Cardiovascular Research Institute, Isfahan, Iran

${ }^{6}$ Isfahan University Of Medical Sciences Isfahan Iran, Psychosomatic Research Center, Isfahan, Iran

\section{Abstract}

Backgrounds: Major cardiovascular risk factors trends have been evaluated less frequently in Middle-East nations. The aim of study was assessing those factors among Iranian persons. Material and method: Methods: 6504 participants aged at least 35 years were recruited for 12 years of longitudinal population based study started in 2001 till 2013 in three phases. Systolic blood pressure (SBP), diastolic blood pressure (DBP), waist circumference (WC), waist to hip ratio (WHR), body mass index (BMI), fasting blood sugar (FBS) and lipid indices including total cholesterol (TC), triglyceride (TG), low-density lipoprotein cholesterol (LDL-C), high-density lipoprotein cholesterol (HDL-C) and TG to HDL-C ratio (TG/HDL-C) were considered risk factors and measured in 2001, 2007 and 2013.

Result: Results: during the follow-up duration, mean ranges of SBP (119.3 \pm 18.7 to128.4 $\pm 17.5 \mathrm{mmHg}, \mathrm{P}<0.001)$, DBP ( $76.7 \pm 11.8$ to82.8 $\pm 12.5 \mathrm{mmHg}, \mathrm{P}<0.001)$, BMI $(27.4 \pm 4.4$ to $28 \pm 4.6 \mathrm{~kg} / \mathrm{m} 2, \mathrm{P}$ $<0.001)$ and FBS $(86.7 \pm 29.6$ to106.5 $\pm 38 \mathrm{mg} / \mathrm{dl}, \mathrm{P}<0.001)$ were increased in both sexes. Favorable downward patterns were observed in terms of TC $(219.5 \pm 51.3$ to200.1 $\pm 40.9 \mathrm{mg} / \mathrm{dl}, \mathrm{P}<0.001)$, TG $(200.2 \pm 110.3$ to155.1 $\pm 85 \mathrm{mg} / \mathrm{dl}, \mathrm{P}<0.001)$, LDL-C $(132.6 \pm 42.4$ to111.7 $\pm 27.8 \mathrm{mg} / \mathrm{dl}, \mathrm{P}<0.001)$ and TG/ HDL-C $(4.5 \pm 2.8$ to3.9 $\pm 2.8, \mathrm{P}$ $<0.001$ ), but HDL-C levels showed less desirable result. WC and WHR patterns revealed controversial results in each gender, but in total population those variabilities were not significant. Conclusion: in spite of desirable trends observed in most lipid indices, other cardiovascular risk factors showed either insignificant or inverse relation. Multiple health strategies required in order to decline the trends of those risk factors showing unfavorable trends.

\section{The association of serum HbA1C level and heart failure severity in systolic heart failure patients with diabetes}

\author{
Vakilian F. ${ }^{1}$, Bijari M. ${ }^{2}$, Naseri H. ${ }^{3}$ \\ ${ }^{1}$ Md Cardiologist Mashhad University Of Medical Sciences, Cardiology, Mashhad, Iran \\ ${ }^{2}$ Student Research Committee Mashhad University Of Medical Sciences, Cardiology, \\ Mashhad, Iran \\ ${ }^{3}$ Md Islamic Azad University Of Medical Sciences, Cardiology, Mashhad, Iran
}

\section{Abstract}

Backgrounds: Heart failure, a major public health problem, causes poor quality of life and increases hospitalization rate. Diabetes mellitus (DM) is associated with both development and progression of heart failure. In this study, we sought to determine the relationship between serum HbA1C levels and severity of heart failure in diabetic patients with systolic heart failure. 
Material and method: This cross-sectional study was performed on 203 patients with heart failure and DM in Mashhad. Demographic data, clinical symptoms and NYHA class were obtained using a questionnaire. Serum HbA1C levels were measured and Echocardiography was performed for all the participants to record ejection fraction (EF).

Result: The HbA1c level was not associated with EF among all the participants and within subgroups based on age, sex, duration of heart failure, heart failure etiology, and number of hospitalization and etiology of hospitalization; However, when patients were subdivided based on NYHA class, there was a significant association between $\mathrm{HbA1C}$ and $\mathrm{EF}$ in patients with NYHA class $\geq 2$ ( $\mathrm{p}<0.05)$. In patients with the duration of DM more than 10 years, $\mathrm{HbA1C}$ levels were significantly different with the maximum levels among patients with $\mathrm{EF}<25 \%$ and the minimum levels among patients with $\mathrm{EF}>35 \%(\mathrm{p}=0.002)$. Moreover, HbA1C levels were higher in patients with EF 25-30\% who didn't have a history of insulin therapy $(\mathrm{p}<0.05)$.

Conclusion: In long standing DM, poor blood glucose control, as measured by $\mathrm{HbA1C}$, is associated with severity of heart failure. Therefore, it is necessary to perform preventive measures in patients with heart failure and DM.

\section{Presentation titled on close follow up for patient underwent PCI for LM}

\section{Alkenzawi H. ${ }^{1}$}

${ }^{1}$ Nasseria Heart Center, Interventional Cardiology, Nasseria, Iraq

\begin{abstract}
Backgrounds: LM artery is the most critical segment of the coronary vascular bed as it perfuses approximately $60 \%$ to $70 \%$ of the myocardium. With advances in PCI technique, stent design and patient selection have made nprotected LM intervention is not inferior or ( no difference) to CABG in term of mortality and MACE but evascularizatiin still higher in LM intervention as compare to CABG

Optimization of intervention to LM ,especially with support of IVUS and OCT to ensure satisfactory results which make routine angiography after unprotected LM intervention is not recommended. However, restenosis might occur which had been manifested as fatal arrhythmia in some cases on top of myocardial infarction and severe acute heart failure.To avoid put these high risk patient after LM intervention in very critical situation we have to put good plan during follow up and closely observing them about there life style modification and inspect their medication inform of dual antiplatlets, high intense statin therapy and other antischemic .

So we will show example of our patient who had been underwent unprotected LM intervention then after presented in disastrous condition and how the control of this condition was difficult. From this we can conclude that the close follow up is mandatory and even control coronary angiography is needed.
\end{abstract}

\section{Prosthetic Valve thrombosis Replacement or Preserving the Valve Single Center Experience}

\author{
Rahmanian M. ${ }^{1}$ \\ ${ }^{1}$ Tehran University Of Medical Sciences, Cardiothoracic Surgey Imam Khomeini Hos- \\ pital, Tehran, Iran
}

\section{Abstract}

Backgrounds: Prosthetic valve thrombosis (PVT) is an ominous complication of patients with mechanical prosthetic valve. We present our surgical experience in cleansing an obstructed prosthetic valve instead of its replacement. Further, we introduce several techniques with safety and good outcome.

Material and method: Between 2011 and 2018, 71 patients including 41 female and 29 male with average age 50.2 \pm 12 years with prosthetic valve malfunction due to PVT( inappropriate for thrombolytic therapy or unresponsive to it) or pannus formation underwent operation. Our strategy was preserving the valve with removing the pannus materials and thrombosis and cleansing the leaflets and their pivots.

Result: our success in preserving the valve was excellent with good result. Only one valve was replaced due to fracture of leaflets. During follow up, two patients developed PVT due to failing to keep the target INR. Overall, there was two death during surgery.

Conclusion: As replacement of a malfunctioned prosthetic valve is probably accompanied by injury to adjacent conduction system or rupture of the $\mathrm{AV}$ groove, preserving the valve is a good option. This method decreases surgical complications and economic load on health system.

\section{Myocardial protection by single dose cardioplegia in on-pump CABG}

\author{
Tavoosi A. ${ }^{3}$

\footnotetext{
${ }^{1}$ Tums Imam Khomeini Hospital, Cardiac Surgery, Tehran, Iran

${ }^{2}$ Tums Imam Khomeini Hospital, Anesthesiology, Tehran, Iran
} \\ ${ }^{3}$ Tums Imam Khomeini Hospital, Cardiology, Tehran, Iran
}

Salehi M. ${ }^{1}$, Bakhshandeh A. ${ }^{1}$, Rahmanian M. ${ }^{1}$, Alemohammad M. ${ }^{2}$, Saberi K. ${ }^{2}$, Sattarzadeh R. ${ }^{3}$,

\section{Abstract}

Backgrounds: Electromechanical activity during cardiac arrest rise oxygen demand during ischemia. In principle, cardioplegia markedly reduces oxygen demand in arrested heart and must be delivered in sufficient quantity to all regions to match myocardial demand. Cold cardioplegic infusion is begun promptly at a flow of $150 \mathrm{~mL} / \mathrm{min} / \mathrm{m} 2$ for 3 minutes in adults; the average adult is given a dose of about $750 \mathrm{~mL}$. Cardioplegic solution is reinfused about every 25 minutes and Reinfusion is given for 30 to 60 seconds.

Material and method: This is a randomized clinical trial between April 2016 and August 2018 in department of cardiovascular surgery, Imam Khomeini Hospital Complex, TUMS on 1023 patients who underwent on-pump CABG, 657 patients as case group (single dose cardioplegia) and 366 patients as control group (multiple dose cardioplegia).

CPB established and conducted as usual, moderate hypothermia and single dose cold blood cardioplegia was the main strategy for myocardial protection in case group and multiple dose cold blood cardioplegia in control group.

The goal of study was to demonstrate there's no statistically significant difference between outcome of patients by single dose versus multiple dose cold blood cardioplegia.

Need to number and dose of inotropes at the time of wean from $\mathrm{CPB}$, new RWMA, LVEF, RV function and all evidence in favor of postoperative acute MI, ICU stay, length of hospital stay and mortality was recorded as the fundamental part of outcome. 
We used cold blood cardioplegia in a ratio of 1:1 in single dose cardioplegia group and Ghazi Tabriz cardioplegia solution in multiple dose group.

Result: In our patients, 689(67.3\%) patients were male and $334(32.7 \%)$ patients were female with mean age of $59 \pm 12$ years. The distribution of LVEF was:

LVEF $<30 \%: 352(34.4 \%)$

LVEF 30-50\%: 430(42\%)

LVEF $>50 \%: 241(23.5 \%)$

The mean number of anastomosis in each patient was $4 \pm 1.6$. The average cross clamp time was $53 \pm 12 \mathrm{~min}$ and pump time was $101 \pm 14 \mathrm{~min}$. The most frequently used inotrops was epinephrine and milrinon. The was no evidence in favor of postoperative myocardial infarction in both groups. IABP was needed in 3 patients in single dose group and in 4 patients in multiple dose group. Mean ICU stay was $2.26 \pm 1.25$ days in single dose group and $2.25 \pm 1.23$ days in multiple dose group which statistically was not significant ( $p$-value $>0.05$ ) . Mean hospital length of stay was: $4.6 \pm 1.02$ days in single dose group and $4.3 \pm 1.2$ days in multiple dose group which statistically was not significant. Mean postoperative LVEF in single dose group was $41 \pm 14.5 \%$ and in multiple dose group was $38.3 \pm 14.9 \%$ which statistically wasn't significant (p-value $>0.05)$. Mortality rate in single dose group was in $18(2.7 \%)$ patients and in 8 (2.1\%) patients in multiple dose group which statistically was not significant $(\mathrm{p}$-value $>0.05$ ). Conclusion: Conduction of on-pump CABG with single dose cold blood cardioplegia seems to be safe and with comparable results with multiple dose cold blood cardioplegia, however more longer and multicenter studies are needed to confirm or contest this hypothesis.

\section{No need to stop taking aspirin and clopidogrel before open-heart surgery}

\author{
Sobhanian K. ${ }^{1}$, Salehi M. ${ }^{1}$, Bakhshandeh A. ${ }^{1}$ \\ Rahmanian M. ${ }^{1}$, Al E Mohamad M. ${ }^{1}$, Bakhtiari A. ${ }^{2}$, \\ Kassani A. ${ }^{3}$ \\ ${ }^{1}$ Tehran University Of Medical Sciencetums, Cardiac Surgery, Tehran, Iran \\ ${ }^{2}$ Dezful University Of Medical Science, Emergency Medicine, Dezful, Iran \\ ${ }^{3}$ Dezful University Of Medical Science, Community Medicine, Dezful, Iran
}

\begin{abstract}
Backgrounds: Patients presenting with acute coronary syndrome (ACS) are treated with aspirin and clopidogrel (Plavix), to prevent mortality and recurrent ischemia. However, those who require open-heart surgery could have increased postoperative bleeding and bleeding-related adverse outcomes. The current guidelines on clinical management differ significantly. This study examined the evidence for clopidogrel and asprin in the treatment of patients requiring open-heart surgery, with a focus on the volume of postoperative blood loss.

Material and method: this cross-sectional study was undertaken involving 702 patients with open-heart surgery between October 2016 and October 2017 (Male=314, 64.12\%). The use of aspirin and plavix in the patients were $20.28 \%(n=142)$ and $14.28 \%$ $(\mathrm{n}=100)$

The blood loss was also measured in three time intervals after the open-heart surgery ( 6 hours, 24 hours and at the time of bleeding cessation). Also, association of post operation blood loss with use of aspirin and plavix were examined by repeated measurement analysis.
\end{abstract}

Result: A total of 702 patients were included in the study. The use of aspirin and plavix in the patients were $20.28 \%(n=142)$ and $14.28 \%(n=100)$. The mean post-operative blood loss in the patients who use of aspirin versus other patients in the time of total loss blood were $732.32 \pm 598.59 \mathrm{cc}$ and $773.49 \pm 780.64 \mathrm{cc}$, respectively $(\mathrm{p}=0.56)$.Also, the mean post-operative blood loss in the patients who use of plavix versus other patients were attained $810.43 \pm 648.82 \mathrm{cc}$ and $757.10 \pm 761.07 \mathrm{cc}(\mathrm{p}=0.52)$. According to finding of repeated measurement analysis, aspirin $(\mathrm{p}=0.62)$ and plavix $(\mathrm{p}=0.93)$ had not significantly associated with postoperative blood loss in 6, 24 hours and in the time of total loss blood.

Conclusion: In our institute we found that aspirin and Plavix usage preoperatively had not significantly associated with postoperative blood loss in 6, 24 hours after operation and in the time of bleeding cessation . aspirin and Plavix usage preoperatively had not significantly associated with higher post-operative blood loss and risk of re-exploration and reduction of blood product usage

Keywords: Open-heart surgery, aspirin, plavix.

\section{Coronary Artery Fistulas A case series of surgical results and clinical characteristics in Iranian population}

\author{
Salahi S. ${ }^{1}$, Azizi Z. ${ }^{2}$, Hosseini S. ${ }^{1}$, Gholampour M. ${ }^{1}$, \\ Baharestani B. ${ }^{1}$, Yaghoubi A. ${ }^{1}$, \\ Alizadeh Ghavidel A. ${ }^{1}$ \\ ${ }^{1}$ Rajaei Cardiovascular Medical Research Center, Cardiac Surgery, Tehran, Iran \\ ${ }^{2}$ Southlake Regional Hospital, Electrophysiology, Toronto, Canada
}

\section{Abstract}

Backgrounds: This study aimed to evaluate the surgical results other than clinical characteristics of patients with coronary artery fistula (CAF) among the Iranian population.

Material and method: This case series consisted of 24 patients with coronary artery fistula who were referred to our tertiary center Rajaei Cardiovascular, Medical \& Research Center for surgical treatment from 2004 to 2018. Patients' demographics, clinical characteristics, and past medical histories were recorded retrospectively, and informed consent was achieved from all patients. Result: Coronary artery Fistula was diagnosed in 24 patients, $11(45.8 \%)$ male with mean age of $35.56 \pm 25$ (0.58-76.33) years with a median of 31 years. The most common symptoms were chest pain in $8(33.3 \%)$ and DOE (dyspnea on exertion) in $7(29.2 \%)$ patients. Valve disorders were presented in 19(79.2\%) patients. According to angiographic results, the most common origins of fistulas were left anterior descending artery(LAD) and right coronary artery (RCA) which were observed in 6 patients( $25 \%)$. Most of the CAFs drained into the pulmonary artery in 10 patients (41.7\%). Concomitant coronary artery bypass graft (CABG) was performed on 8 patients(33.3\%). Mean ICU stay days were 5.9 \pm 5.58 (3-24) days and mean hospital stay days were $23.13 \pm 14.65$ (3-68) days. Mean CPB (cardiopulmonary bypass) time was $110.25 \pm 42.17$ (41-220) minutes. There was no mortality during hospitalization in the present study.

Conclusion: Chest pain and DOE were the most common symptoms in our study. Moreover, our study showed LAD and RCA as the most common origin of fistulas and Pulmonary artery as the most common location of their drainage. Surgical repair can be done safely with excellent results. 


\section{An Investigation of Changes in the Quality of Life of Patients Undergoing Minimally Invasive Mitral Valve Surgery and Surgical Ablation for Atrial Fibrillation Using Ex-Maze III Procedure}

\author{
Nezafati M. ${ }^{1}$, Nezafati P. ${ }^{1}$, Shomali A. ${ }^{1}$ \\ ${ }^{1}$ Mashhad University Of Medical Sciences, Cardiac Surgery, Mashhad, Iran
}

\begin{abstract}
Backgrounds: The aim of this study was to determine the quality of life (QOL) before surgery and 6 and 12 months after surgery in patients with persistent/paroxysmal, and permanent $\mathrm{AF}$, undergoing Radiofrequency (RF) ablation procedure concomitant with Minimally Invasive Mitral Valve surgery (MIMV).

Material and method: From March 2011 to December 2016, 55 patients with relatively same gender and similar age were evaluated. This prospective survey was conducted among 21 consecutive patients with paroxysmal/persistent $\mathrm{AF}$ (15 men) and 34 patients with permanent AF (26 men), aged 40-74 years, undergoing MIMV surgery via right minithoracotomy and direct cannulation, concomitant with RF (using ex-Maze III procedure). For QOL evaluation, 36-Item Short Form Health Survey (SF-36) was used before the operation and 6 and 12 months after the surgery. Results: There were significant differences between the patients of two groups in terms of the average left ventricular ejection fraction (LVEF), left atrium antero-posterior diameter (LAAD), body mass index (BMI), prevalence of systemic hypertension, and previous history of heart failure $(\mathrm{P}<0.001, \mathrm{P}<0.05, \mathrm{P}<0.001$, $\mathrm{P}<0.05$, and $\mathrm{P}<0.05$, respectively). Statistical analysis showed that on average, all eight dimensions of QOL significantly improved 6 and 12 months after ex-Maze procedure in both groups. Conclusion: These results suggest that MIMV surgery concomitant with ex-Maze III procedure for any type of AF is an effective method that improves the QOL of patients in follow-ups. In order to fully evaluate the efficacy of this method, further multicenter, prospective, randomized research with larger sample size is required.
\end{abstract}

\section{Mid-term Results of the First Series of} Minimally Invasive Cardiac Surgeries in Rajaei Cardiovascular Medical and Research Center

\author{
Alizadeh Ghavidel A. ${ }^{1}$, Totounchi Z. ${ }^{2}$, Firouzi A. ${ }^{3}$, \\ Jadbabaei A. ${ }^{1}$, Daliri M. ${ }^{1}$ \\ ${ }^{1}$ Rajaei Cardiovascular Medical And Research Center, Cardiac Surgery, Tehran, Iran \\ ${ }^{2}$ Rajaei Cardiovascular Medical And Research Center, Cardiac Anesthesia, Tehran, Iran \\ ${ }^{3}$ Rajaei Cardiovascular Medical And Research Center, Interventional Cardiology, Teh- \\ ran, Iran
}

\section{Abstract}

Backgrounds: Minimally invasive cardiac surgeries shows postoperative results that are comparable to those of conventional surgeries through the median sternotomy with a shorter ICU and hospital stay, less postoperative pain and better cosmetic results. We aim to present the surgical outcomes of first patients' series in our tertiary center.

Material and method: Between March 2016 and Sep 2018 a total of 50(female:31, male 19) video-assisted minimally invasive cardiac surgeries (ASD closure $=21 \quad M V R=14 \quad$ MV repair=13 ASD + PAPVC=2 MVR+ TV repair $=1$ ) were done for selected cases. Mean age of patients was 36+|- 11 (17-64 years). Patients'outcomes including hospital mortality and survival, postoperative complications, ICU and hospital stay duration are the secondary end point of the study. All surgeries were done using right mini-thoracotomy, peripheral cannulation and antegrade root cardioplegia using Chitwood clamp. The mean total operation time was $339+\mid-74$ minute, mean CPB tine was $125+\mid-52$ minutes (55-320) and the mean aortic cross clamping time was $67+\mid-38 \mathrm{~min}$. (10136).Mean follow up duration was12+/-8 (1-30 months).

Result: Three patient need for conversion to median sternotomy due to bleeding, SAM and low output state. Second run pump was needed in 3 patients due to residual MR, bleeding from left atrial appendage and paravalvular leakage. The in-hospital mortality was occurred in one patient $(2 \%)$ secondary to low output syndrome. We had no late mortality. The mean postoperative transfusion was $271+/-100 \mathrm{ml}(100-300 \mathrm{ml})$ and only one patient needs for re-exploration due to post operativebleeding from intercostal vessels. LCX stenting early after MVR was performed due to the postoperative ST-T changes and coronary artery kinking. Superficial wound infection was seen in one obese lady. We had no other major postoperative complication including AKI, Peri-op MI, CVA and respiratory failure. The mean hospital and ICU stay were $15+\mid-5$ and $2.4+\mid-1$ days consequently. Conclusion: Despite prolonged cardiopulmonary bypass and cross-clamping times, the minimally invasive MVS may be considered as a safe and effective approach with a comparable early and mid-term clinical outcome to the standard median sternotomy with lower ICU stay and post operative bleeding and better cosmetic results.

\section{Constirictive pericarditis mimicking} liver cirrhosis what we should do

\author{
Zarrabi K. ${ }^{1}$, Abtahi F. ${ }^{2}$, Moarref A. ${ }^{2}$, Zamirian M. ${ }^{2}$ \\ ${ }^{1}$ Shiraz University Of Medical Sciences, Cardiac Surgery, Shiraz, Iran \\ ${ }^{2}$ Shiraz University Of Medical Sciences, Cardiology, Shiraz, Iran
}

\section{Abstract}

Backgrounds: In daily medical practice, in every hospital and in every country, many mistakes will happen, which most of them maybe minor \& negligible, but rarely they are so hazardous \& a catastrophe may happen.

In the field of liver transplantation misdiagnosis of constrictive pericarditis (CP) with liver cirrhosis, will be a catastrophe.

Material and method: Since 2007 till 2018, 18 cases of CP, where separated from the huge list of candidates for liver transplantation, which had been referred from whole Iran and neighbouring countries to transplantation center in Namazi hospital (affiliated to Shiraz University of Medical Sciences; SUMS).

The diagnosis of CP confirmed by Joint Committee of Cardiologists, Gastroenterologists \& Cardiac Surgeons, when precise history taking, meticulous physical examination \& proper paraclinical work-ups (CXR, Echocardiography and cardiac catheterization) where indicative of the disease.

Radical subtotal pericardiectomy, performed for all patients. 
Drainage of massive pleural effusion \& ascites was mandatory in some cases. More than $80 \%$ of patients had heavy pericardial calcification, $50 \%$ had AF rhythm \& $20 \%$ had evidence of TB.

Result: All patients except 1, tolerated the procedure \& discharged from hospital within 5-7 days. Dramatic response including cessation of ascites \& lower extremity edema \& improvement of functional class was clearly obvious during the short post-op period of 8-12 weeks.

Conclusion: The most important point was delay in diagnosis which is the leading cause of dense calcification \& ventricular atrophy which makes the prognosis worse.

Internists, Gastroenterologists \& Cardiologists should be aware of this disease entity to avoid a catastrophe.

Keywords: constrictive pericarditis, liver cirrhosis, misdiagnosis

\section{Central versus Peripheral VA ECMO in Critical Heart Failure patients}

\section{Ahmadi Z. ${ }^{1}$, Jahangirifard A. ${ }^{2}$, Sharifi M. ${ }^{1}$, Farzaneghan B. ${ }^{3}$, Sharif Kashani B. ${ }^{4}$, Naghashzadeh F. ${ }^{4}$, Behzadnia N. ${ }^{4}$}

${ }^{1}$ National Research Institiute Of Tuberculosis And Lung Disease, Cardiothoracic Surgery, Tehran, Iran

${ }^{2}$ National Research Institiute Of Tuberculosis And Lung Disease, Cardiac Anesthesia, Tehran, Iran

${ }^{3}$ National Research Institiute Of Tuberculosis And Lung Disease, Intensive Care, Tehran, Iran

${ }^{4}$ National Research Institiute Of Tuberculosis And Lung Disease, Cardiology, Tehran, Iran

\section{Abstract}

Backgrounds: ECMO is the last action that could be done to save the patients with reversible heart and lung disease and also as a bridge to transplant in irreversible heart and lungs. It is also used in the poisonings, viral and infectious diseases, ARDS. But still the mortality is high and its benefit under question. One of the major problems that its efficacy is not determined is route of cannulation in VA ECMO

Material and method: ECMO program was established since 2010 in National Research Institute of Tuberculosis and Lung disease and till September 2018120 patients were undergone ECMO. The following patients excluded from the investigation: 33 lung transplants, 24 lung lavages, 5 complex thoracic surgery operations, and 46 patients due to ARDS, sepsis, poisoning.

22 patients were undergone VA ECMO Result:

\begin{tabular}{lcccc}
\hline $\begin{array}{l}\text { Cannulation } \\
\text { Number }\end{array}$ & $\begin{array}{c}\text { Heart } \\
\text { failure }\end{array}$ & $\begin{array}{c}\text { Pulmonary } \\
\text { Endarterectomy }\end{array}$ & $\begin{array}{c}\text { Post } \\
\text { Cardiotomy }\end{array}$ & Sum \\
\hline Central & 1 & 3 & 6 & 10 \\
Peripheral & 4 & 6 & 2 & 12 \\
\hline
\end{tabular}

The result of ECMO was analyzed:

\begin{tabular}{lccc}
\hline $\begin{array}{l}\text { Cannulation } \\
\text { Number }\end{array}$ & $\begin{array}{c}\text { Duration } \\
\text { (days) }\end{array}$ & Successful & Alive discharge \\
\hline Central & $5 \pm 2$ & $4(40 \%)$ & $2(20 \%)$ \\
Peripheral & $8 \pm 3$ & $8(66 \%)$ & $6(50 \%)$ \\
\hline
\end{tabular}

Conclusion: Athough the Peripheral ECMO seems to be more successful, but due to small number of cases there is no significant difference between 2 groups regarding alive discharge ( $p 0.05$ )
The major cause of death in central ECMO is nonsurgical bleeding. In peripheral ECMO the major cause of death is organ failure, non related to ECMO

It seems that that Peripheral ECMO provides a more safe support for the cardiac patients.

\section{Wilms tumour to the heart how to deal with this challenge}

\author{
Almandil A. ${ }^{1}$, Sultan S. ${ }^{1}$ \\ ${ }^{1}$ Nassirriah Heart Center, Nassirriah Heart Center, Nassirriah, Iraq
}

\section{Abstract}

Backgrounds: Wilms tumour is the most common pediatric malignant renal tumour has a strong sense to metastases into the renal vein and inferior Vena Cava bellow, pre and above hepatic level and reach to right side of the heart, seen in about $1 \%$ of all the cases.

Material and method: A 9 years old girl referred from oncology department with 3 months history of left sided abdominal pain, vomiting, hematuria and fever. Clinical examination revealed fullness in left hypochondrial region and non tender soft immobile mass. Ultrasound, echocardiography and contrast enhancement CT scan revealed well defined mass replacing the lower part of left kidney extending to renal vein and through IVC to the heart. A preoperative chemotherapy was started and the comprehensive evaluation and preparing for surgery by multidisciplinary team done.

Result: Median sternotomy and extended laparotomy, left nephroctomy done then with aid of cardiopulmonary bypass and cardiac arrest the thrombus like tumour retrieved along venotomy and right atriotomy and sent for histopathology. Conclusion: A review of others similar published studies indicated that multimodal therapy with removal of the tumour associated with good outcome of wilms tumour management

\section{Prevalence of Anemia In Patients Undergoing Cardiac Surgery in Rajaie Heart Center And Need for Transfusion of Blood During Operation Regarding to Hemoglobin Level}

\author{
Sadeghi A. ${ }^{1}$, Farasatkish R. ${ }^{1}$, Azarfarin R. ${ }^{1}$, \\ Ziyaeifard M. ${ }^{1}$, Faritous Z. ${ }^{1}$, Hadipoorzadeh F. ${ }^{1}$ \\ ${ }^{1}$ Rajaie Heart Center, Anesthesiology, Tehran, Iran
}

\section{Abstract}

Backgrounds: Bleeding occurs during and after cardiac surgery, resulting in postoperative anemia. If patients have preoperative anemia, the need for blood transfusion increases. Transfusion is associated with several complications.

Material and method: In this study, patients entered to the operating room prior to the anesthesia induction an arterial catheter was placed and hemoglobin level was determined and given the amount of hemoglobin ( $\mathrm{Hb})$, anemia level is determined as: $\mathrm{Hb}$ less than $8 \mathrm{~g} / \mathrm{dl}$ : Severe anemia; Hb between 8 to $10 \mathrm{~g} / \mathrm{dl}$ : Moderate anemia and Hb 10-12 (for women) and 10 -13 (for men): Mild 
anemia.The study also looked at the age, sex, type of surgery and weight of patients and for the association of anemia with the above mentioned variables.Also, during surgery amount of blood transfusion to the patient was evaluated. The need for transfusion was evaluated according to Hb level.

Result: In this study, 306 patients were studied in three months period. The means hemoglobin level of patients was $13.1 \mathrm{~g} / \mathrm{dl}$ (12.08-14.2) and the mean hematocrit was 39.5\%(36.17-42.15).In this study, $32.4 \%$ of patients had anemia $(\mathrm{Hb}<12$ for women and $\mathrm{Hb}<13$ for men).According to the anemia classification, 90.9\% of anemic patients had Mild anemia, 8.1\% moderate anemia and $1 \%$ severe anemia. of 306 patients, $68.6 \%$ did not need to receive packed red blood cells(PC). In this study, of 207 patients were not included in the anemia group 44(21.2\%) patients received PC due to surgical bleeding. But, of 99 cases that were anemic, 52(52.52\%) patients in this group needed PC.

Conclusion: About one third of our patients had preoperative anemia. The anemic patients needed to be transfused 2.5 times more than those without anemia.

\section{- Comparison of the malposition between midpoint and Lateral point approaches for subclavian vein catheter placement in patients undergoing cardiac surgery}

\section{Tarbiat M. ${ }^{1}$, Bakhshaei M. ${ }^{1}$, Salimbahrami S. ${ }^{1}$, Khorshidi H. ${ }^{2}$}

${ }^{1}$ Clinical Research Development Unit Of Farshchian Heart Center, Department Of Anesthesiology, Hamadan, Iran

${ }^{2}$ Clinical Research Development Unit Of Farshchian Heart Center, Department Of Thorax Surgery, Hamadan, Iran

\footnotetext{
Abstract

Backgrounds: Percutaneous subclavian vein catheterization is one of the most common invasive procedures performed in cardiac surgery. Malpositioning of central venous catheter inserted into subclavian vein is a known complication. The aim of this study was to compare the malposition between midpoint and lateral point approaches via the infraclavicular technique in patients who undergoing cardiac surgery.

Material and method: This prospective, randomized clinica trial was performed in 308 patients. The patients were randomly assigned to one of the two groups (midpoint or Lateral point approach). In midpoint approach, inserting the needle 2 to 3 $\mathrm{cm}$ inferior to the midpoint of the clavicle. In Lateral point approach, inserting the needle 1 to $2 \mathrm{~cm}$ lateral to the midclavicular line. The technique of cannulation for both groups was infraclavicular approach. The malposition of catheterizetions was compared in the both insertion point groups

Result: In nine of 308 (8 in midpoint approach and 1 in latera point approach) catheters placed through subclavian vein were misplaced. In midpoint approach, seven catheter tips were placed in the ipsilateral internal jugular vein and in one, catheter formed a loop around itself over the right subclavian vein (5.19\%), but at Lateral point approach, one catheter tip was located in the contralateral subclavian vein (0.64\%). The overal malposition of the catheter tip in both groups was $2.92 \%$. There was a significant difference betweenmidpoint and Lateral point approaches $(\mathrm{P}=0.015)$.

Conclusion: Compared with the midpoint approach, lateral point approach resulted in fewer catheter tip misplacements
}

via the infraclavicular technique for subclavian vein catheterization in patients undergoing cardiac surgery.

\section{The factors that affect blood and blood products usage in CABG}

\author{
Saberi K. ${ }^{1}$, Salehi M. ${ }^{2}$, Bakhshandeh A. ${ }^{2}$, \\ Rahmanian M. ${ }^{2}$, Alemohammad M. \\ ${ }^{1}$ Tums Imam Khomeimi Complex, Anesthesiology, Tehran, Iran \\ ${ }^{2}$ Tums Imam Khomeimi Complex, Cardiac Surgery, Tehran, Iran
}

\section{Abstract}

Backgrounds: In spite of the evidence suggesting a significant morbidity associated with blood transfusions, the use of blood and blood products remain high in cardiac surgery. To successfully minimize the need for blood transfusion, a systematic approach is needed. The aim of this study was to investigate the influence of different surgery strategies, on-pump vs off-pump, on postoperative bleeding complications and the need for blood transfusions during perioperative period.

Material and method: We examined 371 patients undergoing isolated non-emergent coronary artery bypass (CABG) surgery. We submitted the most recent patients who received 0-4 units of RBC transfusion during the index admission. Patient and disease characteristics, intra-operative practices, and percentage of cases receiving RBC transfusions were collected. Region-specific transfusion rates were calculated, after adjusting for pre- and intra-operative factors.

Result: Average PC transfusion was higher amongst patients with $\mathrm{HB}$ level $<10$ and in female patients.

Conclusion: The most predictive factor for blood transfusion was patients low HB level.

\section{The Effect of Dexmedetomidine on Hemodynamic Responses during Tracheal Extubation}

\author{
Karami N. ${ }^{1}$, Mahoori A. ${ }^{1}$ \\ ${ }^{1}$ Urmia University Of Medical Sciences, Department Of Anesthesiology, Urmia, Iran
}

\section{Abstract}

Backgrounds: Dexmedetomidine is a alpha-2 adrenoreceptor agonist that has effective sedation with less cardiovascular unstability and respiratoty depression and may beuseful fot extubation.

Material and method: Fifty women entered to study in two groups randomly and were intubated intratracheally. Ten minutes before end of surgery, 0.8 microgram $/ \mathrm{kg}$ of dexmedetomidine was infused over ten minutes in study group and normal saline in control group a placebo. Blood pressure, heart rate and saturation of oxygen were recorded in 0,1,2,3 and 5 minutes after extubation. Also, sedation index was recorded.

Result: In this study heart rate, systolic and dyastolic pressure in patient with infusion of dexmedtomidine were lower than control group significantly as well as nausea and vomiting.

Conclusion: Infusion of dexmedetomidine 10 minutes before extubation facilitate extubation and lead to hemodynamic stability. 
An assessment of the renal function with pulsatile perfusion during the proximal graft by using cardiac contraction in CABG surgery

\author{
Amouzegar Zavareh S. ${ }^{1}$, Lak M. ${ }^{1}$ \\ ${ }^{1}$ Baqiatallah Medical University, Cardiac Anesthesia, Tehran, Iran
}

\begin{abstract}
Backgrounds: Short-term pulsatile perfusion during cardio pulmonary bypass has shown higher protection for renal physiology, following in standard preservation of glomerular filtration and reduce renal tissue damage.

Material and method: in this randomized clinical trial study 70 patientswho underwent coronary artery bypass graft (CABG) surgery divided in two groups: pulsatile and non-pulsatile groups. we transferred continuous blood flow to pulsatile blood flow during proximal graft by using cardiac contraction. Patients' laboratory tests such as BUN, creatinine were checked preoperatively and at the arrival of the ICU-OH and 24 and 48 hours after admission. Result: in both case and control had significantly increased in BUN and creatinine. Increased creatinine in the first and second days postoperative was significantly higher in the control group. and also BUN increased on the second day in the control group was significantly higher than Case group.

Conclusion: Despite the rise in BUN and creatinine in our study, there were no cases of renal failure in patients. a further study is needed to examine the effects of pulsatile flow upon renal function and its long-term significance.
\end{abstract}

\section{The relationship between fluid balance and the incidence of post- operative acute kidney injury during and 24 hours after CABG surgery}

\author{
Alavi S. ${ }^{1}$, Ziyaeifard M. ${ }^{1}$, Babaei T. ${ }^{1}$, \\ Bakhshandeh $\mathrm{H}^{2}{ }^{2}$
}

${ }^{1}$ Rajaie Cardiovascular Medical And Research Center, Anesthesiology, Tehran, Iran ${ }^{2}$ Rajaie Cardiovascular Medical And Research Center, Epidemiology, Tehran, Iran

\begin{abstract}
Backgrounds: About 30\% of patients undergoing cardiac surgery experience acute kidney injury (AKI). Increased fluid intake after coronary artery bypass graft (CABG) surgery can be a cause for renal failure in patients. The purpose of this study was to determine the relationship between fluid overload and serum creatinine changes in patients undergoing CABG surgery. Material and method: 130 patients undergoing CABG surgery were entered into this study using Cardiopulmonary Bypass (CPB) at Shahid Rajaie Cardiology Center. The fluid balance number for each patient was calculated 24 hrs after surgery, and each patient was classified into one of these three groups:1) Patients with a fluid balance of less than $2000 \mathrm{ml}$; 2) Patients with fluid balance of 2000 to $3000 \mathrm{ml}$; 3) Patients with a fluid balance of more than $3000 \mathrm{ml}$. Serum creatinine changes and renal failure were studied in these three groups.

Result: After multivariate analysis of the factors affecting the incidence of AKI in the studied patients, it was found that there
\end{abstract}

was a significant relationship between inotrope and AKI incidence ( $\mathrm{P}=0.001,75.1875$ to 847.5 , 95\% C.I , 664/89, OR). However, there is no significant relationship between the incidence of AKI and the high fluid balance.

Conclusion: There are many interactions between renal function and fluid balance during a cardiovascular-pump. According to the results of this study, the high fluid balance in patients has a minor effect on creatinine, and creatinine changes have not caused AKI.

\section{Comparison of modified tight control versus conventional glucose control methods among diabetic patients undergoing cardiac surgery}

\author{
Javaherforoosh Zadeh F. ${ }^{1}$
}

${ }^{1}$ Ahvaz Jundishapur University Of Medical Sciences Ahvaz Iran, Cardiac Anesthesia, Ahvaz, Iran

\section{Abstract}

Backgrounds: About 30-40\% of patients who are undergoing Coronary Artery Bypass Graft patients have diabetes mellitus or metabolic syndrome and are at risk for hyperglycaemia events.

The aim of this study was comparison of the outcome of patients undergoing open cardiac surgery by glucose control in two ways: modified tight versus conventional method.

Material and method: This Open-labelled, randomized control trial was conducted on 75 diabetic patients, aged 18-70 years with ASA II- III undergoing open heart surgery from Shahid Faghihi Hospital, Shiraz ,Iran. Patients were divided into modified tight control (Blood Sugar maintained between 100-120 mg/dl) and conventional method (Blood Sugar $\leq 200 \mathrm{mg} / \mathrm{dl}$ ). Mortality, sternal wound infection, duration of mechanical ventilation, cardiac arrhythmia, cerebrovascular attack and acute renal failure are considered as primary outcomes and length of ICU staying has been determined as secondary outcomes. Complications after30 days of surgery were recorded by surgeon.student's t test, chi-square and repeated measurement tests

Result: Although the trend of change in blood glucose during surgery and in ICU was not significantly different between groups, Blood Sugar measurement showed a significant difference at the different times $(\mathrm{P}<0.001)$. In terms of primary postoperative outcomes the two groups were not significantly different while, hypokalemia was the most prominent side effect in the 34 patients of modified tight control compared with 8 patients in conventional control group $(\mathrm{P}<0.001)$. In review of patients after one month, sternal wound infection was one patient in group MTGC versus 7 case in group CGC $(\mathrm{P}<0.05)$ which was statistically significant.

Conclusion: Using a modified tight control of blood glucose level to a level that is close to normal values during cardiac surgery may reduce episodes of hypoglycaemia and hyperglycaemic complications.

\section{Non-surgical treatment of right} ventricular muscle band in a child right ventricular outlet tract stenting

\author{
Meraji M. ${ }^{1}$, Zolfigol A. ${ }^{1}$, Jafari F. ${ }^{1}$ \\ ${ }^{1}$ Rajaie Cardiovascular Medical And Research Center, Pediatric Cardiology, Tehran, \\ Iran
}




\section{Abstract \\ Backgrounds: Routinely right ventricular outlet tract (RVOT) stenosis corrected surgically. Percutaneous stenting of RVOT mostly done for conduit stenosis or as palliation therapy for native RVOT obstruction in symptomatic infants with complex congenital heart diseases (CHD) such as tetralogy of fallout. There are some reports about native RVOT stenting in an adult with noncomplex CHDs. \\ Material and method: Herein we describe native RVOT stent ing in a child with a history of ventricular septal defect (VSD). A four years girls with a history of VSD that had pulmonary ar- tery band at 6-month-old and surgical VSD closure at 15 months old, showed post surgically subpulmonary stenosis and severe tricuspid regurgitation in echocardiography. In angiography RV muscle band was obvious, RV pressure was supra systemic and there was $70 \mathrm{mmHg}$ pressure gradient between RV body and pul- monary artery. \\ Result: RVOT stenting was done with cook formula stent size $10 / 20 \mathrm{~mm}$. pressure gradient decreased to $20 \mathrm{mmHg}$ after the procedure. \\ Conclusion: RVOT stenting provides safe and effective pallia- tion for infants with complex CHDs especially when surgery is not possible. Is RVOT stenting an effective treatment for right ventricular muscle band? Long-term follow up is needed. \\ The effect of Atorvastatin on mortality and complications of pulmonary embolism short and long- term follow-up}

Jenab Y. ${ }^{1}$, Vahidi H. ${ }^{1}$, Mortazavi S. ${ }^{1}$

${ }^{1}$ Tehran Heart Center Tehran University Of Medical Sciences Tehran Iran, Cardiology, Tehran, Iran

\begin{abstract}
Backgrounds: Venous thromboembolism, the $3^{\text {rd }}$ most common cardiovascular disease, could be fatal in the acute phase. Several studies have shown the association between atherothrombosis and pulmonary embolism and some have confirmed the positive effect of statins on this disease.

Material and method: Patients with pulmonary embolism were divided into four groups based on the history of receiving or not receiving Atorvastatin prior to admission $(\mathrm{Hx})$ and during admission (AD): 1. Hx-AD- $(\mathrm{n}=240)$ 2. $\mathrm{Hx}-\mathrm{AD}+(\mathrm{n}=227) 3 . \mathrm{Hx}+\mathrm{AD}+$ $(n=73) 4$. Hx+AD- $(n=16)$. The 4th group was excluded from the analysis. These groups were compared with respect to the mortality and pulmonary embolism complications during hospitalization course. Statin users ( $\mathrm{n}=250$ ), were also compared with non-statin users $(\mathrm{n}=270) 1$ year later.

Result: The rate of mortality and/or adverse events during admission was $11.3 \%, 15 \%$ and $5.5 \%$ in the 1 st, 2nd and the $3 \mathrm{rd}$ groups, respectively. The adjustment analysis showed significant effect of statin on reducing the adverse events (OR:0.44 95\% CI: 0.14-1.37; $\mathrm{P}=0.024)$. A total of 30 deaths and 4 recurrent emboli was detected during one-year follow-up. After adjustment for possible confounders, no significant difference was found in adverse events between statin and non-statin users after 1-year follow-up.

Conclusion: Lowest in-hospital adverse event was seen in $\mathrm{Hx}+\mathrm{AD}+$ group which might imply the protective anti-inflam-
\end{abstract}

matory effect of statin. Highest adverse events in Hx-AD+ group, might suggest that statin would be just effective in acute phase of the disease.

\section{The predictive role of Insulin-like growth factor-1 and Pregnancy- associated plasma protein $\mathrm{A}$ biomarkers in acute coronary syndrome and its related morbidities}

\author{
Mehrpooya M. ${ }^{1}$, Zebardast J. ${ }^{2}$, Malek Kandi \\ M. ${ }^{3}$, Hasan Zade K. ${ }^{3}$, Arabloo M. ${ }^{3}$, Asad Zade L. ${ }^{3}$ \\ ${ }^{1}$ Tehran University Of Medical Scince, Interventional Cardiology, Tehran, Iran \\ ${ }^{2}$ Tums, Educational Affair, Tehran, Iran \\ ${ }^{3}$ Uromia University Of Medical Science, Imam Khomeini Hospital of Naghade, \\ Naghade, Iran
}

\section{Abstract}

Backgrounds: Pregnancy-associated plasma protein A (PAPPA) is a metalloproteinase, plays role in atherosclerotic plaque destabilization. In recent studies, Insulin-like growth factor -I (IGF-1), has been considered as a mediator of atherosclerosis. PAPP-A and IGF-1 may be rose in acute coronary syndromes (ACS). The present study tried to assess role of IGF-1 and PAPP-A biomarkers in spectrum of ACS.

Material and method: The serum level of IGF-1, PAPP-A and troponin I was determined in 121 consecutive patients with ACS. Result: In patients with acute ST segment elevation myocardial infarction (STEMI), all of these three biomarkers were significantly higher than patients with unstable angina $(p=0.008$ for IGF-1, $\mathrm{p}=0.004$ for PAPP-A and $\mathrm{p}<0.0001$ for troponin I). Receiver operator characteristic (ROC) curve analysis showed PAPP-A to be a good discriminator between patients with STEMI and patients with unstable angina. Optimum cut-off value for PAPP-A was found to be $95.6 \mathrm{ng} / \mathrm{ml}$.

Mean level of IGF-1 in patients with renal failure was significantly higher than those without this morbidity (137.87 \pm 35.13 versus $105.18 \pm 46.94, p=0.003)$. No difference was found in the level of PAPP-A between the patients with and without renal failure $(142.39 \pm 47.46$ versus $99.41 \pm 33.13, \mathrm{p}=0.265)$. Serum troponin $\mathrm{I}$ level wasn't significant $(\mathrm{p}=0.8)$ regarding presence or absence of renal failure $(0.84 \pm 0.29$ versus $0.91 \pm 0.15)$.

Conclusion: PAPP-A could be novel biomarkers for identification of patients with STEMI also risk stratification in patients with ACS

\section{Nonpharmacologic treatments} alone are enough to prevent neurally mediated syncope A 3 year follow up study

Dehghan B. ${ }^{1}$, Sabri M. ${ }^{2}$

${ }^{1}$ Pediatric Cardiovascular Research Center

${ }^{2}$ Cardiovascular Research Institute, Isfahan University of Medical Science

\section{Abstract}

Backgrounds: Recurrences are common in neurally mediated 
syncope. The aim of this study is the evaluation of the effectiveness of nonpharmacologic treatments alone in preventing of syncope relapse.

Material and method: 70 patients (age 5-20 years) with neurally mediated syncope were enrolled. Thirty patients received pharmacologic therapies along with nonpharmacological methods, and 40 patients received just nonpharmacological treatments then followed them for 36 months. The incidences of different outcomes were analyzed with descriptive statistics using percentages.

Result: The recurrence rate of syncope was significantly higher in pharmacological group than in nonpharmacological group in each period of the followup $(\mathrm{P}<0.001)$.

Conclusion: Nonpharmacologic treatment is very effective in the prevention of syncope relapses and can be a substitute for pharmacologic drugs in the initiation of treatment and if done correctly.

\section{Challenging case of perimembranous VSD closure}

\author{
Molaei A. ${ }^{1}$ \\ ${ }^{1}$ Tabriz University Of Medical Sciences, Pediatrics, Tabriz, Iran
}

\begin{abstract}
Backgrounds: Ventricular septal defect (VSD) is the most common congenital heart defect requiring therapeutic intervention. Membranous defect is the most common type of VSDs. It's over 50 years, VSD closure is done through surgery and sternotomy, using patch and Cardiopulmonary bypass. But the probability of serious complications are the limitations of this method.

Trans catheter VSD closure was started from about 2 decades ago, with some advantages and disadvantages.

Material and method: In this report, we introduce a 17-years old female with membranous VSD that was candidated for transcatheter VSD closure by device. After catheterization and hemodynamic study and angiography in appropriate views the defect was occluded by device associated by more challenges.

Result: the defect size was about $14 \mathrm{~mm}$ with aneurismal pouch formation. The pulmonary arterial pressure was normal. The defect was occluded by muscular VSD occluder size $16 \mathrm{~mm}$ successfully but with serious challenges. There was no significant residual shunt post procedure.

Conclusion: Like any invasive procedure to achieve a therapeutic approach to the success of treatment and minimize the complications of intervention, regarding the recommended principles are required.
\end{abstract}

\section{Experience of coronary AV fistula closure by PFM coils}

\author{
Mehdizadegan N. ${ }^{1}$, Amoozgar H. ${ }^{1}$ \\ ${ }^{1}$ Shiraz University Of Medical Sciences, Pediatric, Shiraz, Iran
}

\section{Abstract}

Backgrounds: Coronary AV fistulas are rare but important congenital problems among children.55\% of cases are from right coronary artery and $45 \%$ from the left coronary arteries. These fistulas are from coronary arteries to atriums, right ventricle ,coronary sinus and superior vena cava.

Material and method: 12 cases were included in this study from 2010 till 2016. Seven cases were girl and five cases were boy. Age of patients were between 2 months to 10 years. All the cases were symptomatic and were diagnosed by physical examination, ECG and finally echocardiography and then referred to catheterization laboratory for percutaneous closure by PFM coils.

Result: All of the patient had only coronary AV fistula as isolated problem. There were no death among the patients. We have 3 cases with complications, 2 patients had residual flow with the first inserted coil among the fistula and closed completely with using another coil. One patient complained of chest pain after closure of fistula without ECG change or rising troponin level and chest pain subsided one day after that and went home without more chest pain. Among this experience 10 patients closed successfully without residual flow or major complications.

Conclusion: PFM coils can be used safely as devices for successful closure of coronary AV fistulas with minor or rare complications even in small babies.

\section{- Heart function and Remodeling after successful stenting of Coarctation of Aorta in children}

\author{
Amoozgar H. ${ }^{1}$, Rashid H. ${ }^{2}$, Edraki M. ${ }^{3}$, \\ Mohammadi H. ${ }^{4}$, Ajami G. ${ }^{3}$, Borzoee M. ${ }^{3}$ \\ ${ }^{1}$ Shiraz University Of Medical Sciences, Cardiovascular Research Center, Shiraz, Iran \\ ${ }^{2}$ Shiraz University Of Medical Sciences, Pediatric Department, Shiraz, Iran \\ ${ }^{3}$ Shiraz University Of Medical Sciences, Cardiovascular Research Center, Shiraz, Iran \\ ${ }^{4}$ Shiraz University Of Medical Sciences, Neonatal Research Center, Shiraz, Iran
}

\section{Abstract}

Backgrounds: There are not enough studies that have investigated the results of stent implantation for coarctation of the aorta (COA) in pediatric age group. This investigation evaluated the heart function and remodeling after successful stenting in children.

Material and method: The study was performed from April 2016 till April 2017 on patients with COA ( $<18$ years old). Demographic data, clinical examination results and echocardiographic parameters were reported. All statistical analyses were performed with the SPSS version 22.

Result: Mean age at the time of stenting was $10.44 \pm 3.93$, and mean follow-up was $2.57 \pm 1.40$ years (1-6 years). 14 patients ( $77.8 \%$ ) were male. Measurement of thoracic aorta diameters revealed that mean transverse arch, isthmus and distal arch diameter with its Z-scores were $1.40 \pm 0.43$ (Z score $=-1.50 \pm 1.33), 1.17 \pm 0.28$ ( $Z$ score $=-0.72 \pm 1.43)$ and $1.21 \pm 0.42(Z$ score $=-0.94 \pm 3.46)$. Mean systolic blood pressure at the time of follow-up was $122.5 \pm 17.70 \mathrm{mmHg}$ and mean diastolic blood pressure was $81.25 \pm 9.57$. Significant correlation existed between IVSd value and transverse arch diameter $(\mathrm{P}=0.023, \mathrm{r}=0.564)$, and also distal arch diameter $(\mathrm{P}=0.039$, $\mathrm{r}=0.521)$. There was also a significant correlation between LVIDd and measured transverse $(\mathrm{P}=0.007, \mathrm{r}=0.648)$ and distal arch diameter $(\mathrm{P}=0.034, \mathrm{r}=0.521)$. Instead, the correlation between $\mathrm{E} / \mathrm{A}$ value and IVSs $(\mathrm{P}=0.023)$ and IVSs $\mathrm{Z}$ score $(\mathrm{P}=0.044, \mathrm{r}=0.480)$ was significant. There was a significant correlation between LVPW pa- 
rameter and Doppler measurement of gradient at the site of stent $(\mathrm{P}=0.024, \mathrm{r}=0.621)$ measured at the time of follow-up.

Conclusion: Based on the results endovascular stenting to treat CoA appears to be a safe and efficient method. LV hypertrophy and diastolic dysfunction did not regress in significant number of patients after successful stenting.

\section{- Rehabilitation of the Pulmonary Artery in Tetralogy of Fallot with Unilateral Absent Pulmonary Artery}

\author{
Edraki M. ${ }^{1}$, Keshavarz K. ${ }^{1}$ \\ ${ }^{1}$ Shiraz University Of Medical Sciences, Pediatric Cardiology, Shiraz, Iran
}

\begin{abstract}
Backgrounds: Unilateral absence of one pulmonary artery (PA) is a well-known congenital heart disease called De Buckes syndrome that may occur in isolation or with other cardiac disorders like tetralogy of Fallot (TOF); and untreated disorder may cause unilateral pulmonary hypoplasia.

Some patients who have no collaterals or has closed PDA, do not show any pulmonary artery branch in imaging modalities. Therefore these cases whose investigations did not demonstrate the good size and operable PA, underwent direct connection of the right ventricle to the healthy pulmonary artery, and spend their life with one lung.

Material and method: We present 4 of these cases with impression of TOF absent PA whom we struggled to find and open their absent PA.

Our patients were $1,12,30$ and 60 months old.

Pulmonary three dimensional CT angiography showed some small hypoplastic sparse artery branches, with no remnant of the PA at the hilum, therefore they were supposed to be operated with one pulmonary artery.

We performed cardiac catheterization and right ventriculography in anterior-posterior and left anterior oblique views showed good size one PA, but no opacification of another PA.

Also ascending aorta injection demonstrated right aortic arch and one blind-ending stump of possibly PDA toward the lung. Then we inflated one bare coronary stent into the PDA successfully to enlarge the PA.

Result: We fortunately were able to find and open a nice path between aorta and apparently absent pulmonary artery Conclusion: Sometimes we might be successful to open an apparently absent PA.
\end{abstract}

\section{Evaluation of etiology and time between tetralogy of Fallot total correction and pulmonary valve replacement through 5 years ago in Tabriz Shahid Madani hospital}

\author{
Ghaffari S. ${ }^{1}$, Samadi M. ${ }^{1}$, Jamei Khosroshahi \\ A. ${ }^{1}$, Molaie A. ${ }^{1}$, Moradian M. ${ }^{1}$, Abdi Oskouei M. ${ }^{1}$ \\ ${ }^{1}$ Tabriz University Of Medical Sciences, Cardiovascular Research Center, Tabriz, Iran
}

\section{Abstract}

Backgrounds: This study aims to determine the time interval between the total correction of tetralogy of Fallot (TOF) and pulmonary valve replacement (PVR).

Material and method: In a retrospective cohort study, patients with TOF underwent complete surgical repair and PVR surgery in Tabriz Shahid Madani Hospital from 2012 to 2017. The patients were divided into 3 groups: A: patients that their pulmonary valve was restored by transannular patch method (35.29\%), B: patients that in addition to transannular patch, monocusp method (14.71\%), C: patients with commissurotomy procedure.

Result: In 34 patients who had been correction of TOF in the age range of 1- 5 years, the highest rate of severe pulmonary insufficiency before PVR (62.50\%) was seen. In other patients undergoing PVR, replacement pulmonary valve stenosis and insufficiency was seen too. In patients needing PVR, on average, age of groups A, B, C was $77 \pm 5.12,6.40 \pm 1.517 .53 \pm 6.78$ years, receptively. Group B had the shortest interval between the complete correction of TOF and the PVR.

Conclusion: Based on the findings of this study, it is deduced that the longer time interval between the complete correction of TOF and PVR has the lower mortality rate of this disease; therefore, it is better to avoid PVR as much as possible; so patients are less involved side effects after surgery. Also Patients with mild to moderate pulmonary insufficiency underwent valve replacement surgery due to stenosis of the pulmonary valve.

\section{Relation of preoperative diastolic pulmonary gradient and pulmonary compliance with early post- operative mortality after cardiac transplantation}

\author{
Taghavi S. ${ }^{1}$, Amin A. ${ }^{1}$, Naderi N. ${ }^{1}$, Taban Sad- \\ eghi M. ${ }^{2}$
}

${ }^{1}$ Rajaee Heart Center, Cardiovascular, Tehran, Iran
${ }^{2}$ Tabriz Cardiovascular Research Center, Cardiovascular, Tabriz, Iran

\section{Abstract}

Backgrounds: Orthotropic heart transplantation (HTx) remains as gold standard for these End stage patients. Pulmonary hypertension (PH) occurs in up to70 \% of patients with advanced heart failure which classified as "passive" or "reactive".We reviewed the relationship between preoperative pulmonary hemodynamic and early post-transplant mortality.

Material and method: In a retrospective study Between 2007 through May 2018, a total of 125 heart transplant, 72 adult patients with heart failure underwent Orthotropic HTx procedures at Rajaie Cardiovascular Medical and Research Center. All adult patients were enrolled in this trial.

Pulmonary hemodynamic indices werepulmonary vascular resistance (PVR), trans-pulmonary gradient (TPG), Diastolic pulmonary gradient (DPG) and pulmonary artery compliance (PAC). End points were 30-day and one year survival.

Student's t test for paired parameters and Fisher's exact test for relationship of hemodynamic parameters and outcome after transplantation were used. 
Result: Sixty four study patients (16 female, 48 male) were age ranged from 14 to 60 years (mean 28.2 \pm 9.7 ).There was no statistically significant correlation between PVR, PVRI, TPG, DPG, PAC and 30-day post-transplant mortality. Thirty-day survival was similar between low TPG or DPG and high TPG or DPG groups. But the 1 year mortality rate after transplantation was significantly higher in patients with higher PVR, TPG and DPG.

Conclusion: Pre-operative pulmonary hemodynamic parameters such as PVR, TPG and DPG can predict 1year, but not 30 days post-transplant mortality. DPG Cannot predict 1-year post-transplant mortality belter than TPG.

\section{Facial nerve palsy in Kawasaki disease}

\section{Rajaei S. ${ }^{1}$}

${ }^{1}$ Clinical Research Development Center Of Children Hospital Hormozgan University Of Medical Sciences, Pediatrics, Bandar Abbas, Iran

\section{Abstract}

Backgrounds: Kawasaki disease is one of the most common vasculitis of childhood that primarily involved medium size arteries throughout the body but rarely complicated by facial nerve palsy. We report a 4.6 month old female presenting with prolonged fever who did not respond to broad spectrum antibiotics and subsequently diagnosed with Kawasaki disease. Although initial echocardiogram was normal, the repeated one after 10 days showed dilated bilateral (right and left) coronary arteries. At the same times she developed acute leftsided peripheral facial palsy. Intravenous immunoglobulin was started and fever was resolved after 36 hours. At 2 and 6 weeks post-discharge, echocardiography demonstrated gradually resolution of her coronary aneurysm Facial palsy markedly recovered after 2 weeks and completely after about 8 weeks. Facial nerve palsy is one of the rare neurological manifestations of Kawasaki disease and resolves spontaneously and completely in surviving patients, but it's indicates an increased risk of coronary artery involvement and may require additional anti-inflammatory agents and more vigilant echocardiography.

Keywords: Kawasaki disease-Facial nerve palsy- Coronary artery

\section{Prevalence and risk Factors of Attention Deficit hyperactivity disorder After Artrial Switch Operation in Neonatal Period}

\author{
Peiravian F. ${ }^{1}$, Akhavan Z. ${ }^{1}$, Toubaee S. ${ }^{2}$, \\ Amirghofran A. ${ }^{3}$ \\ ${ }^{1}$ Faculty Of Medicine Kazerun Branch Islamic Azad University, Pediatrics Ward, Kaze- \\ run, Iran \\ ${ }^{2}$ Faculty Of Medicine Kazerun Branch Islamic Azad University, Psychiatry Ward, Kaze- \\ run, Iran \\ ${ }^{3}$ Shiraz University Of Medical Sciences, Cardiac Surgery Ward, Shiraz, Iran
}

\section{Abstract}

Backgrounds: Complex cardiac surgeries using cardiopulmo- nary bypass ( $\mathrm{CPB}$ ) during early life may affect normal neurobehavioral development in these patients including attention deficit hyperactivity disorder (ADHD). Aim of this study is to assess the prevalence and risk factors of ADHD after arterial switch operation (ASO) during first month of life.

Material and method: 60 survivors of ASO in neonatal period from one center were included. 47 patients completed the study. Control group consisted of 50 healthy children. Both groups were divided to two subgroups; G1, below 2.5 years old and G2, above 2.5 years. For G1, CSBS DP Infant-Toddler Checklist questionnaire and for G2 patients, DSM-IV questionnaire were used for diagnosis of ADHD. Correlation of demographic data and perioperative factors with development of ADHD was investigated.

Result: There were 38 males and 9 females. Mean age was 49 months (8-192) in cases and 50.3 (10-194) in control group. In our study the prevalence of ADHD in case group was approximately 2.5 times the control group, $48 / 9 \%$ vs. $20 \%$. ( $\mathrm{p}=0.0001$ ) In operated patients $40 / 4 \%$ had hyperactivity disorder, $0 \%$ attention deficit and $8 / 5 \%$ combined disorder, compared with $10 \%, 4 \%$ and $6 \%$ in control group. Risk factors for development of ADHD after neonatal ASO were younger age at the time of surgery, lower temperature during $\mathrm{CPB}$, longer aortic cross clamp (ACC) and CPB time, and higher age at the time of interview. ( $\mathrm{p}=0.0001,0.045,0.006,0.001,0.0001$ respectively) Cut off points were operation age less than 13 days old, CPB temperature less than $29^{\circ} \mathrm{C}$, ACC time above 80 and CPB time above 112 minutes, and interview age above 24 month.

Conclusion: In this study, prevalence of ADHD in survivors of neonatal ASO was high. Related symptoms were more apparent by increasing age. Improving CPB techniques and operation time may decrease incidence of this complication.

\section{A Large Venovenous Collateral from Renal Vein as a Cause of Desaturation in Fontan Circulation}

\author{
Shabanian R. ${ }^{1}$, Navabi Shirazi M. ${ }^{2}$, Dadkhah M. ${ }^{1}$ \\ ${ }^{1}$ Childrens Medical Center Tehran University Of Medical Sciences, Department Of Pe- \\ diatric Cardiology, Tehran, Iran \\ ${ }^{2}$ Childrens Medical Center Tehran University Of Medical Sciences, Department Of Pe- \\ diatric Cardiac Surgery, Tehran, Iran
}

\section{Abstract}

Backgrounds: Oxygen saturation in Fontan circulation is in the low 90s. Significant desaturation may be due to residual large fenestration, intrapulmonary arteriovenous malformation and abnormal drainage of systemic venous to the pulmonary venous atrium. These collaterals will be closed surgically or by intervention in symptomatic cyanotic patients.

A 24-year-old male with history of ECTCPC Fontan circulation evaluated for progressive desaturation (SpO2=68\%). Contrast echo from an IV-line in leg showed microbubbles in single ventricle without visualizing in Fontan conduit in favor of collateral vein drainage to systemic circulation. For more evaluation, CT angiography was performed which showed a prominent venovenous collateral originating from the left renal vein that was connected to the left atrium via a dilated ascending vein into a tuft of tortuous veins. Angiography and catheterization revealed a mean of less than $15 \mathrm{mmHg}$ in Fontan pathway and ruled out the presence of a large fenestration. The ascending vein was occluded by an Occlutech ASD device size of $15 \mathrm{~mm}$ successfully. Control injection showed the complete closure of collateral without any extravasation. 
The patient O2 Saturation was elevated up to $90 \%, 15$ minutes after device deployment.

After Fontan circulation, increase in central venous pressure cause recanalization of obliterated embryonic veins. These venovenous collaterals decompress higher pressure veins to lower pressure pulmonary veins which drain to atrium and cause cyanosis.

\section{The effect of protocol weaning from ventilator on the duration of mechanical ventilation in patients with acute kidney injury after open heart surgery}

\author{
Asadzadeh H. ${ }^{1}$ \\ ${ }^{1}$ Shahid Rajaei Hospital, Nursing, Tehran, Iran
}

\begin{abstract}
Backgrounds: The risk of acute kidney injury (AKI) incandidates for coronary artery bypass graft (CABG) surgery usually is highlighted. Also postoperative AKI remains a serious complication of CABG and is associated with the long weaning time from mechanical ventilation in critically ill patients. This study aimed to determine the effect of discontinuation protocol on the weaning duration from mechanical ventilation in patients with AKI after open hart surgery hospitalized in intensive care units (ICUs) of Shahid Rajaei Hospital.

Material and method: It was a randomized clinical trial. One hundred patients who had been diagnosed with acute kidney injury after CABG were assigned to either experimental $(n=50)$ or control $(n=50)$ group. The experimental group was weaned by a special protocol by trained nurses and the control group was weaned as usual. Data was analyzed by descriptive and inferential statistics (t-test, chi-square and Pearson correlation) using SPSS (v.22).

Result: The findings showed that the mean weaning duration in experimental group is significantly lower than the control group $(\mathrm{p}=0.002)$. Serum urea and creatinine concentrations in the experimental group was significantly lower than the control group ( $\mathrm{p} \leq 0.04)$. There were also a significant positive corolation between Serum urea and creatinine concentrations and weaning duration $(\mathrm{p} \leq 0.016)$.

Conclusion: The results showed that using a structured guideline and weaning tool shortens the duration of weaning in ICUs and also increases renal function comparing routine methods

\section{The effect of using standard checklist on nursing handover status in cardiac care units of Afshar hospital in Yazd}

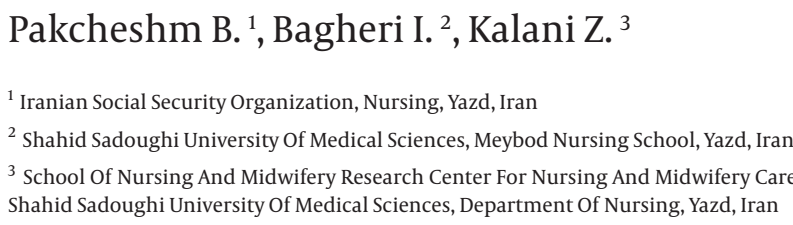

\section{Abstract}

Backgrounds: Handover is defined as transfer of information between health care members.One-fifth of negative side effects in patients and $80 \%$ of the medical errors are due to poor handover.Absence of standard handover protocol in healthcare settings resulted in providing unnecessary information,losing essential information, and committing medical errors.Therefore, this study was attempted to investigate the effect of using standard checklist on nursing handover status in cardiac care unit Material and method: This semi-experimental study was performed in 3 stages in Afshar Hospital located in Yazd, Iran,in 2017. In total,546 handovers were investigated with the presence of 24 nurses. In the first stage,282 nursing handovers were recorded in two CCUs of the hospital. In the second stage, nurses got familiar with the ISBAR checklist and were encouraged to use it for one week. In the third stage, 282 nursing handovers were recorded again.Frequency of data provision was investigated before and after the intervention.Data were analyzed using descriptive statistics and paired t-test

Result: Frequency of data provision in handovers was $86.9 \%$ in patient identification domain,75.1\% in patient's current situation domain, $52.8 \%$ in patient's clinical background domain,59.9\% in status of systems assessment domain, and $92.9 \%$ in recommendations domain before the intervention.However, after the intervention,a significant increase in 5 domains was observed in a way that frequency of data provision in nursing handovers was $100 \%, 94 \%, 80.1 \%, 92.2 \%$, and $100 \%$ in aforementioned domains, respectively $(\mathrm{P}<0.001)$

Conclusion: Information transferring based on a standard checklist and a specific framework improved the nursing handover.Therefore,training of medical members and students about the standard handover in hospitals and universities is suggested

\section{The Relationship between Health Literacy and Adherence to Treatment in Cardiac Patients}

\author{
Khaleghparast S. ${ }^{1}$, Maleki A. ${ }^{2}$, Salesi M. ${ }^{1}$, \\ Ghanbari B. ${ }^{3}$, Maleki M. ${ }^{1}$
}

${ }^{1}$ Rajaie Cardiovascular Medical And Research Center, Iran University Of Medical Sciences, Tehran, Iran

2 Department Of Anesthesiology Hazrat Rasool Akram Hospital, Iran University Of Medical Sciences, Tehran, Iran

${ }^{3}$ Gastrointestinal And Liver Disease Research Center Firoozgar Hospital, Iran University Of Medical Sciences, Tehran, Iran

\section{Abstract}

Backgrounds: The aim of this study was to investigate the health status of cardiovascular patients and their relationship with the patients' adherence status to their drug therapy.

Material and method: The present study was a descriptive-correlational study. The research population included the patients referred to these clinics. 400 participants were selected based on inclusion and exclusion criteria from the research population. The sampling method was simple random sampling. The instrument consisted of two parts. The first part evaluated demographic information and the second part was based on the combination of two standard instruments of cardiovascular literacy and adherence to treatment, which were used after being modified and reassessed in terms of validity and reliability. The obtained information was analyzed by the SPSS Version 19.

Result: Spearman test indicated that general health literacy has a significant relationship with treatment $(r=0.31, P<0.001)$. This correlation was also observed in relation to sub-scales of percep- 
tion of health $(\mathrm{r}=0.27)$ and health behavior by adherence to treatment $(r=0.29)(P<0.001)$. If the participants received information through their physicians, they showed a significant relationship between health literacy (in all sub-scales) and adherence to treatment $(\mathrm{P}<0.001)$.

Conclusion: The results showed that there is a high correlation between health literacy and adherence to treatment. Therefore, considering the importance of cardiovascular diseases, as well as the importance of health literacy and its relevance to adherence to treatment, it is suggested that TVs located in the clinics of the center, broadcast the doctors' training films.

\section{Neglected lead in electrocardiogram interpretation}

\author{
Mohsenabadi M. ${ }^{1}$, Mohsenipour Foumani A. ${ }^{1}$ \\ ${ }^{1}$ Iranian Social Securityorganization, Nursing, Tehran, Iran
}

\begin{abstract}
Backgrounds: In spite of the progress of medical science every day and the emergence of new methods for the investigation and diagnosis of many disorders and cardiac disorders, the 12 leads electrocardiogram is an easy, inexpensive, fast and accessible diagnostic method for the diagnosis of cardiac disorders that can be very useful. Of the electrocardiogram leads, aVR has traditionally received less attention in clinical evaluation of the ECG. Lead aVR, one of the 12 electrocardiogram lead, is frequently ignored in clinical medicine. In fact, many clinicians refer to the 12-lead electrocardiogram (ECG) as the 11-lead ECG, noting the commonly held belief that lead aVR rarely offers clinically useful information. Lead aVR has multiple clinical applications and is a useful tool for interpreting ECGs. The present study discusses instances with pictorial examples in which lead aVR provides valuable clinical information and makes a case for close attention being paid to this 'forgotten lead'.

Material and method: This article has been reviewed by searching in databases and expressing the role of aVR lead in the diagnosis of cardiac disorders and syndromes that all of them have been discussed with electrocardiograms.

Result: Nevertheless, aVR - the forgotten lead - can be a useful tool in the diagnosis and prognosis of many clinical syndromes. Conclusion: Careful attention to aVR lead during evaluation of the ECG can aid in the diagnosis of acute Heart diseases such as LMCA or proximal LAD occlusion, affecting timing and type of therapy, and predicting prognosis in patients with acute myocardial infarction. It is recommended that ECG readers pay attention to the aVR lead in the interpretation of the electrocardiogram, because it is very helpful in detecting some syndromes and heart disease.
\end{abstract}

\section{Assessing serum fibrinogen} and fibrin degradation products FDP changes in hypothermia and normothermia in patients undergoing cardiopulmonary bypass

\author{
Banakar Y. ${ }^{1}$, Ghodrati M. ${ }^{1}$ \\ ${ }^{1}$ Shiraz University Of Medical Sciences, Anesthesia, Shiraz, Iran
}

\section{$\underline{\underline{\text { Abstract }}}$}

Backgrounds: Today, the use of cardiopulmonary bypass (CPB) with hypothermia for heart surgeries is relatively common and is performed more than 200,000 times a year in the United States. Physiological activity of the body when the patient is on cardiopulmonary bypass is very different from normal physiological activity, which most patients will have relatively serious adverse effects. In this study, we assessed the changes in serum fibrinogen and fibrin degradation products (FDP) in hypothermia and noromothemia in patients undergoing cardiopulmonary bypass.

Material and method: Our study is a cross-sectional clinical trial. The research population comprisedf all adult patients undergoing cardiac surgery. The method of sampling was random (permutation blocks). 80 patients were divided into two groups: hypothermia (fibrinogen $=40$ and FDP $=22$ ) and noromothemia (fibrinogen $=40$ and FDP $=22$ ). $2 \mathrm{cc}$ of arterial blood samples were taken and placed in tubes containing tri sodium citrate two times. The first sample was taken in both groups before the injection of heparin and the start of CPB, and the second one in the hypothermic group before the onset of warming and in the normothermic group after opening aortic valve.

Result: Patients included 22 women and 58 men with the mean age of $61.43 \pm 12.64$ years old. The mean temperature in the hypothermia group was $32.33 \pm 1.44$ and $35.33 \pm 0.71$ in the normothermic group. The mean of bleedin in first 24 hours was $652 \pm 256.31$ cc in the hypothermia group and $645.12 \pm 203.27 \mathrm{cc}$ in the normothermic group, which did not show significant differences between two groups $(P=0.717)$. Serum levels of fibrinogen decreased significantly in both groups $(\mathrm{P}=<0.001)$. changes Serum levels FDP was similar in both groups(hypothermia $\mathrm{P}=0.778$ and normothermia $\mathrm{P}=0.412$

Conclusion: The during cardiac surgery in hypothermia and neurotropic conditions rate of fibrinogen decreased $25 \%$; this decrease seems to be due to hemodilution. FDP levels were similar in both groups. Fibrinogen check can be a good prognosis for fibrinogen deficiency bleeding disorder. And give the surgeon a favorable view for proper hemostatic treatment and prevention of bleeding

\section{Hyperthermic Intra-Operative Chemotherapy for Management of Peritoneal Carcinomatosis with a cardiopulmonary bypass machine}

\author{
Banakar Y. ${ }^{1}$, Ghodrati M. ${ }^{1}$ \\ ${ }^{1}$ Shiraz University Of Medical Sciences, Anesthesia, Shiraz, Iran
}

\section{Abstract}

Backgrounds: HIPEC is standard of care in many countries for patients with peritoneal cancer now, that is challenging and complicated. HIPEC is a type of hyperthermic treatment, combined with Cytoreductive (CRS) surgery used to treat advanced abdominal cancer. The purpose of this study is to describe the use of cardiopulmonary bypass for HIPEC and the results obtained in improving patients and its technical and financial aspects compared to the HIPEC device.

Material and method: During two year, from 2016 to 2018, 60 patients Suffering to cancer under CRS and HIPEC with a cardiopulmonary bypass machine, in Central Hospital of Shiraz, entered 
the study and examined and intervened after receiving written consent. Patient characteristics, type of primary cancer, duration of surgery, type of operation, duration of HIPEC, type of chemotherapy substance, morbidity including: early postoperative Complications re-surgery, 30 days postoperative mortality and mortality of one to four years, installation costs, Repair, Maintenance and technical support device were collected and recorde Result: A total of 60 patients, 39 women (65\%) and 21 men (35\%) with an average age of $17 \pm 50.36 .6 \%$ of patients developed a postoperative complication. The overall mortality was 12 cases (20\%), 30 days postoperative mortality, 5 cases (10\%), and overall survival of one and two years were $89 \%$ and $78 \%$. The mean temperature of the operation was $41 \pm 0.7$. The average ICU stay and HIPEC procedure is "cost-effective" with a average reduction in costs. Conclusion: The cardiopulmonary bypass machin is an efficient, safe and low cost alternative to the HIPEC device. The cost of consumables for each patient is lower in cardiopulmonary bypass machin. This treatment is effective if patients selected with high precision, and the perfusion team, with the knowledge of this process and permanent monitoring, can manage acceptable standards for doing this.

\section{Recommendations for Standards of Monitoring and Alarms during Cardiopulmonary Bypass}

\author{
Rahimi H. ${ }^{1}$ \\ ${ }^{1}$ Mashhad University Of Medical Sciences, Cardiac Surgery, Mashhad, Iran
}

\section{Abstract}

Backgrounds: It is accepted that monitoring during the operative period reduces risks for patients. In 1988 the Association of Anaesthetists of Great Britain and Ireland first published Recommendations for Standards of Monitoring during Anaesthesia and Recovery. Within these recommendations it was recognised that additional monitoring may be required during cardiopulmonary bypass.

The aim of this document is to determine standards for monitoring and alarms during cardiopulmonary bypass.

Material and method: This includes monitoring for the onset of and weaning from cardiopulmonary bypass, for example confirmation of anticoagulation and ventilation of the lungs. These standards are for use in conjunction with the Society of Perfusionists of Great Britain and Ireland Standards of Practice document 1 and local protocols.

Result: The recommended monitors and alarms that should be used during cardiopulmonary bypass are considered by the Society of Perfusionists of Great Britain and Ireland, the Association of Cardiothoracic Anaesthetists and the Society of Cardiothoracic Surgeons of Great Britain and Ireland to be the minimal monitoring requirements during cardiopulmonary bypass. All centres undertaking cardiac surgery involving cardiopulmonary bypass should plan to institute these standards of monitoring and alarms by the 1st January 2003. It is accepted that special clinical circumstances, for example emergency surgery or failure to insert a urinary catheter, may on some occasions preclude complete monitoring. There may be additional monitoring requirements during cardiopulmonary bypass for paediatric patients.

Conclusion: The safe conduct of cardiopulmonary bypass is a joint responsibility of surgeons, anaesthetists and clinical perfusionists and requires a high level of communication between the team members. At all times during the conduct of cardiopulmonary bypass a surgeon, an anaesthetist and a clinical perfusionist must be present in the operating room.

\section{Serum levels of IL-6 IL-10 and TNF- $\alpha$ renal function biochemical parameters and patients' outcomes in pediatric cardiopulmonary bypass surgery}

Gorjipour F. ${ }^{1}$, Totonchi Z. ${ }^{2}$, Tirgarfakheri K. ${ }^{2}$, Maziar Gholampour Dehaki M. ${ }^{2}$, Hosseini S. ${ }^{2}$, Sadeghi T. ${ }^{3}$, Mortazian M. ${ }^{2}$, Mehrabanian M. ${ }^{4}$, Gorjipour F. ${ }^{2}$

\footnotetext{
${ }^{1}$ Iranian Scientific Society Of Extracorporeal Technology Rajaie Cardiovascular Medical And Research Center Iran University Of Medical Sciences, Iranian Scientific Society Of Extracorporeal Technology, Tehran, Iran

2 Rajaie Cardiovascular Medical And Research Center Iran University Of Medical Sciences, Rajaie Cardiovascular Medical And Research Center Iran University Of Medica Sciences, Tehran, Iran

${ }^{3}$ Tehran Medical Sciences Branch Islamic Azad University, Tehran Medical Sciences Branch Islamic Azad University, Tehran, Iran

${ }^{4}$ Tehran Heart Center Tehran University Of Medical Sciences, Tehran Heart Center Tehran University Of Medical Sciences, Tehran, Iran
}

\section{Abstract}

Backgrounds: Cardiopulmonary bypass ( $\mathrm{CPB}$ ) causes detrimental effects on remote organs due to inflammatory response. One of these organs is kidney that is frequently affected by cardiac surgery. Acute kidney injury (AKI) is a common post-CPB complication increasing the morbidity and mortality. Post-CPB inflammatory response may contribute to remote organ dysfunction. In the present study, we investigated the relation between cytokines including IL-6, IL-8, IL-10, and TNF- $\alpha$, and renal function tests such as creatinine and BUN.

Material and method: 91 patients were enrolled for elective cardiac surgery with $\mathrm{CPB}$ after informed consent.

Result: our findings support that there is a direct correlation between cytokines including IL-6, IL-8, IL-10, and TNF- $\alpha$ and CPB time, duration of operation and ICU stay. Longer CPB time was associated with higher IL-8 at cross-clamp removal and 24 hours post-ICU as well as higher IL-10 at declamp time. Higher IL-6 at declamp time was directly correlated with higher postoperative BUN. IL-8 level after anesthesia induction was directly correlated with ICU stay duration. Higher blood IL- 6 and TNF- $\alpha$ levels following 24 hours of transfer to ICU was associated with longer mechanical ventilation time.

Conclusion: Higher circulatory proinflammatory cytokines level is associated with adverse outcomes such as increased ICU stay and longer mechanical ventilation time. It is also correlated with unfavorable biochemical parameter of renal function, BUN. Findings hint that proper control of the inflammatory response is vital for the control of unfavorable clinical and pathological outcomes.

The Influence Of Mean Arterial Blood Pressure During Cardiopulmonary Bypass On Post Acute Kidney Injury AKI In Hypertensive Patients

\author{
Foroutan M. ${ }^{1}$, Fatahi M. ${ }^{1}$, Ziyaeifard M. ${ }^{2}$, Alavi S. ${ }^{2}$
}


${ }^{1}$ Rajaei Cardiovascular Medical Research Center, Perfusion, Tehran, Iran

${ }^{2}$ Rajaei Cardiovascular Medical Research Center, Cardiac Anesthesiology, Tehran, Iran

\section{Abstract}

Backgrounds: Over a period of time, kidney blood vessels will be damaged in those patients suffering from hypertension. The disease causes reduction of blood flow to the kidney tissues which may be resulted in kidney failure. Maybe, people suffering from hypertension have not been stricken with some degrees of kidney failure; however, following a hypo perfusion shock such as undergoing a bypass pumping and creation of favorable conditions, renal dysfunction would be probable. So, in the research, effect of mean arterial pressure during cardiopulmonary bypass (CPB) has been studied on occurrence of cardiac surgery-associated acute kidney injury (CSA-AKI) in patients suffering from hypertension. Material and method: Present observational futuristic study has been performed on 120 high risk patients, having records of high blood pressure and being candidates for open heart surgery, through usage of bypass pumping equipment. Patients have been divided into three groups based on their MAPs (mean arterial pressure) during bypass operation. Mean arterial pressure of first, second, and third groups have been lower than $60 \mathrm{mmHg}$, between 60 to $70 \mathrm{mmHg}$, and higher than $70 \mathrm{mmHg}$, respectively. Clinical outcomes being studied included cardiac surgery-associated acute kidney injury (CSA-AKI) (RIFLE class), creatinine level, glomerular filtration rate, and urinary output up to 24 hours after the surgery.

Result: Data related to the 120 case-patients have been analyzed, in general. There has been no difference between the groups, in terms of demographic information. Prevalence of acute kidney injury in preliminary course after cardiac surgery has been different among the groups. Acute kidney injury has been occurred in 6 patients (15\%) of the first group, 5 patients (12.5\%) of the second group, and 2 patients $(5 \%)$ of the third group. There have been no differences in terms of creatinine level, glomerular filtration rate, and urinary output during 24 hours after cardiac surgery, among these groups; however, changes in creatinine level and glomerular filtration rate have been significant among the first and second groups of patients.

Conclusion: The study showed that MAP (mean arterial pressure) level during cardiopulmonary bypass (CPB) pumping directly affects occurrence of acute kidney injury in patients, after open heart surgery; and, high risk patients such as those suffering from hypertension have to be constantly monitored during bypass pumping, in terms of their MAPs. MAP drop to lower than $70 \mathrm{mmHg}$ has to be prevented in these patients.

\section{- Effect of Adenosine Fast Cardioplegic Arrest Induced on Troponin Levels After On-pump bypass surgery}

\section{Bagherinasab M. ${ }^{1}$}

${ }^{1}$ Shiraz University Of Medical Science, Anesthesiology, Shiraz, Iran

\section{Abstract}

Backgrounds: Cell damage is not avoidable with current cardioplegic solutions. The effect of adenosine administration on reducing cardiac injury is controversial. The aim of this study is to evaluate the effect of adenosine fast cardioplegic arrest on high sensitive cardiac troponin I after heart valve surgery.
Material and method: Fifty-six patients underwent heart valve surgery using conventional approach through median sternotomy. They were classified into two groups, group I $(\mathrm{n}=28)$ patients received $0.25 \mathrm{mg} / \mathrm{kg}$ adenosine into the aortic root just after aortic cross-clamping and before infusion of the cold hyperkalaemic crystalloid cardioplegia via antegrade route and group II $(n=28)$ who received cold crystalloid hyperkalaemic cardioplegia without adenosine. Cardiac troponin I was measured preoperatively and on postoperative days 0,3 and 7.

Result: Adenosine significantly reduced arrest time. Postoperative high sensitive cardiac troponin I increased significantly in both groups compared to the preoperative levels and the rise continued till postoperative day 3. Troponin levels were significantly lower in the adenosine group compared to the control at all measurements. The clinical outcomes were non-significant different between groups.

Conclusion: Using adenosine in inducing fast cardioplegic arrest in heart valve surgery after aortic cross clamp and prior to infusion of the cold cardioplegia had significantly decreased postoperative cardiac troponin levels which was used as a proxy for cellular injury compared to the control group.

\section{Cardio-pulmonary bypass during pregnancy}

\author{
Azizi B. ${ }^{1}$ \\ ${ }^{1}$ Shahid Faghihi Hospital, Cardiac Surgery, Shiraz, Iran
}

\section{Abstract}

Backgrounds: Cardiac surgery carried out on cardiopulmonary bypass (CPB) in a pregnant woman is associated with poor neonatal outcomes although maternal outcomes are similar to cardiac surgery in nonpregnant women.

Material and method: Most adverse maternal and fetal outcomes from cardiac surgery during pregnancy are attributed to effects of CPB.The CPB is associated with uteroplacental hypo perfusion due to a number of factors, which may translate into low fetal cardiac output, hypoxia and even death.

Result: Better maternal and fetal outcomes may be achieved by early preoperative optimization of maternal cardiovascular status, use of perioperative fetal monitoring, optimization of CPB, delivery of a viable fetus before the operation and scheduling cardiac surgery on an elective basis during the second trimester. Conclusion: In this article we will review the perfusionist management strategies during cardiopulmonary bypass in a pregnant woman.

Keywords: Cardiac surgery, Cardiopulmonary bypass, pregnancy

\section{- The effect of Delnido and Custodiol cardioplegia on peri-operation clinical outcomes in patients with Tetralogy of Fallot who undergoing cardiopulmonary bypass}

\author{
Heidari Nia S. ${ }^{1}$ \\ ${ }^{1}$ Iranian Scientific Society Of Extracorporeal Technology, Rajaie Cardiovascular Medi- \\ cal And Research Center, Tehran, Iran
}




\begin{abstract}
Backgrounds: Delnido and Custodiol are used for myocardial protection during cardiopulmonary bypass in cardiac surgery by surgeons. Damages to myocardium may happen due to ischemia/reperfusion injury and enhanced inflammatory response.Cytokines are important determinants of inflammatory responses.
\end{abstract}

Material and method: In a randomized controlled clinica trial,we investigated two groups of patients receiving Delnido and Custodiol in terms of clinical outcomes. Patients in both groups received a standard anesthesia protocol. We assess Troponin-I level before operation and ICU entrance. Thus we measure serum lactate level before and after operation, we also measure coronary sinus lactate level after opening the cross-clamp. Patients' blood chemistry parameters; demographic data and other background information were recorded in questionnaire and were used for statistical analysis. Analysis was performed using SPSS.

Result: Frequency of male patients was $71.42 \%$ in Delnido and $57.14 \%$ in Custodiol,age was $17.71 \pm 9.53$ versus $15.28 \pm 7.45$.differences between two groups were not significant and Blood chemistry parameters and blood gas analysis were similar in both groups, but sodium, potassium, and chloride level in Delnido and Custodiol were(140_4.65_114) versus (134_3.7_108) respectively,during $\mathrm{CPB}(\mathrm{p}>0.05)$ and also coronary sinus lactate level were significantly in higher range in Custodiol group $(\mathrm{p}<0.05)$.

Conclusion: In this study we concluded that both types of cardioplegia are effective enough to protect myocardium during $\mathrm{CPB}$,it is recommended to limit the usage of custodiol in patients with electrolyte disturbances.

\section{Guidelines for Temperature Management during Cardiopulmonary Bypass}

\author{
Yadegary M. ${ }^{1}$, Bayat R. ${ }^{1}$, Hamadani K. ${ }^{1}$, Karami \\ A. ${ }^{1}$, Abrahimi A. ${ }^{1}$, Rezvan M. ${ }^{1}$, Mohamadi F. ${ }^{1}$, \\ Eazti M. ${ }^{1}$
}

${ }^{1}$ Zanjan University Of Medical Sciences, Critical Care Nursing Department, Zanjan, Iran

\begin{abstract}
Backgrounds: we conduct a narrative review article to improve our understanding of Temperature Management during CPB that including 1) optimal site for temperature monitoring, 2) avoidance of hyperthermia, 3) peak cooling temperature gradient and cooling rate, and 4) peak warming temperature gradient and rewarming rate.

Material and method: we searched online databases such as Science direct, Embase, MEDLINE, CINAHL, IranMedex, Magiran and SID in order to retrieve Temperature Management during $\mathrm{CPB}$. Search terms were cardiopulmonary bypass or perfusion with body temperature or body temperature regulation or rewarming or hypothermia, induced/ instrumentation or hypothermia, induced/methods. Finally we assess 15 full text articles. Result: Optimal Site for Temperature Measurement: although optimal core temperature site has not been reported, but the intravascular and intracorporeal location of a PA catheter probably the best access for core temperature recording. Jugular bulb (JB), temperature is recognized as being the best indicator
\end{abstract}

of cerebral temperature.

Avoidance of Hyperthermia: arterial outlet temperature exceeding $370 \mathrm{C}$ during $\mathrm{CPB}$ is an independent predictor of acute kidney injury.

Peak Cooling Temperature Gradient and Cooling Rate: Severe brain injury when an arterial-venous gradient of $20 \mathrm{oC}$ was used for cooling and significantly less injury when the cooling gradient was Limited to $4 \mathrm{oC}$.

Peak Warming Temperature Gradient and Rewarming Rate: slow rewarming avoidance of excess cerebral oxygen extraction and jugular venous desaturation, improved maintenance of the relationship between cerebral blood flow and the cerebral metabolic rate of oxygen, increased time for better distribution of heat. Conclusion: Oxygenator arterial outlet blood temperature is recommended to be used as a surrogate for cerebral temperature measurement during CPB . PA catheter or NP temperature recording is reasonable for weaning and immediate post bypass temperature measurement.Surgical teams should limit arterial outlet blood temperature to $<37 \mathrm{o}^{\mathrm{C}}$ to avoid cerebral hyperthermia.Temperature gradients from the arterial outlet and venous inflow on the oxygenator during CPB cooling should not exceed $10 \mathrm{oC}$ to avoid generation of gaseous emboli . Peak warming temperature gradient and rewarming rate

Temperature gradients from the arterial outlet and venous inflow on the oxygenator during CPB rewarming should not exceed $10 \mathrm{oC}$ to avoid outgassing when warm blood is returned to the patient.Rewarming when arterial blood outlet temperature ${ }^{3} 30 \mathrm{oC}$. To achieve the desired temperature for separation from bypass, it is reasonable to maintain a temperature gradient between arterial outlet temperature and the venous inflow of $£ 4 \mathrm{oC}$. To achieve the desired temperature for separation from bypass, it is reasonable to maintain a rewarming rate of $£ .5 \mathrm{oC}$ min. Rewarming when arterial blood outlet temperature is $<30$ oC. To achieve the desired temperature for separation from bypass, it is reasonable to maintain a maximal gradient of $10 \mathrm{oC}$ between arterial outlet temperature and venous inflow.

\section{- Clinical and laboratory results of adding Albumin Versus Fresh Frozen Plasma in Priming Solution of CPB in Pediatric Patients}

\section{Hosseinzadeh M. ${ }^{1}$, Gholampour Dehaki M. ${ }^{1}$, Gorjipour F. ${ }^{1}$, Taghizadeh A. ${ }^{2}$, Taiyari S. ${ }^{2}$}

\footnotetext{
${ }^{1}$ Rajaie Cardiovascular Medical And Research Center, Cardiac Surgery, Tehran, Iran

${ }^{2}$ Children Medical Center Tehran University of Medical Sciences, Pediatric Cardiac Surgery, Tehran, Iran
}

\section{Abstract}

Backgrounds: The decrease in blood oncotic pressure following hemodilution during Cardiopulmonary Bypass in cardiac surgery results in fluid shift and organ dysfunction. The present comparative study evaluated the effect of adding Albumin and Fresh Frozen Plasma (FFP) in Primer solution of a cardiopulmonary bypass system (CPB) on clinical and laboratory consequences in Congenital Cardiac Surgery candidates.

Material and method: In this Clinical Trial study, 83 pediatric patients' candidate for the elective surgical repair for congenital heart disease were recruited and assigned to two groups of FFP and Albumin by random. The patients' mean arterial blood pressure, central venous pressure, diuresis, arterial blood gases, and 
arterial blood lactate were measured and recorded in eight-time points. Laboratory tests including ESR, CRP, BUN, and Creatinine were measured before surgery and in ICU.

Result: The mean age of the patients in groups of Alb and FFP were 7.3 and 10.6 months in sequence. Inflammatory markers as ESR and CRP were significantly higher in the group of FFP (P $<0.05)$. Kidney functioning markers as BUN and Creatinine were remarkably lower in Alb group $(\mathrm{P}<0.05)$. No statistically significant differences in hemodynamic variables, the volume of the red blood cell (RBC) transfused, need for Inotrope injection were observed between two groups $(\mathrm{P}>0.05)$.

Conclusion: The patients, with Fresh Frozen Plasma in prime solution, had higher inflammatory markers. However, Albumin added into the prime helped the patients to have a better kidney function.

\section{The survival rate and complications after congenital heart surgery in children under membrane oxygenation machine outside the body ECMO}

Taghavi S. ${ }^{1}$, Mahdavi M. ${ }^{2}$, Totonchi Z. ${ }^{2}$, Jalali A. ${ }^{2}$, Skeikhi B. ${ }^{3}$, Gorjipour F. ${ }^{3}$, Tasmeh S. ${ }^{2}$

${ }^{1}$ Mazandaran University Of Medical Sciences, Mazandaran University Of Medical Sciences, Mazandaran, Iran

${ }^{2}$ Rajaie Cardiovascular Medical And Research Center Iran University Of Medical Sciences, Rajaie Cardiovascular Medical And Research Center Iran University Of Medical Sciences, Tehran, Iran

${ }^{3}$ Rajaie Cardiovascular Medical And Research Center Iran University Of Medical Sciences, Iranian Scientific Society Of Extracorporeal Technology, Tehran, Iran

\section{Abstract}

Backgrounds: Despite the increasing experience and advanced technological developments in membrane oxygenation machine outside the body (ECMO), the mortality rate of patients undergoing treatment remains still high. Considering the increase in congenital heart surgeries and the increased demand for ECMO to reduce mortality and improve the outcome of congenital heart surgery in children. The aim of this study was to determine the rate of survival and complications after congenital heart surgery in children under the protection of Venous - Arterial's membrane oxygenation machine outside the body (ECMO).

Material and method: In this retrospective study 25 patients undergoing surgery for congenital heart disease at Shahid Rajaee Cardiovascular Research and Treatment Center were studied in 2016-2018. These patients after surgery were located under membrane oxygenation machine outside the body (ECMO), because of heart or respiratory problems, failures in separation from the bypass pulmonary artery (CPB). all patients 'information was extracted from the patients' files according to a prescriptive checklist. data were analyzed by SPSS software using the chi-square and Fisher's exact test.

Result: Of the 25 patients, were 13 males (52\%) and 12 females (48\%). Their mean age was $8 \pm 0.68$ (at least 2 months and maximum 14 years). death was observed in $56 \%$ of patients (14 patients). In the patient were been Neurological complications in $44 \%$ of patients (11 patients), requiring dialysis in $40 \%$ of patients (10 patients), and gastrointestinal complications in $12 \%$ of patients (3 patients).

Conclusion: According to the results of this study, more than half of the patients died and the most complications after surgery were related to neurological complications and the need for dialysis among them.
Zero-Balance Ultrafiltration of the Priming Blood Modifies the Priming Components and Improves the Clinical Outcome in Infants Undergoing Cardiopulmonary Bypass A Randomized Controlled Trial

Niknam S. ${ }^{1}$, Gholampour M. ${ }^{2}$, Azarfarin R. ${ }^{3}$, Salehzade A. ${ }^{1}$

${ }^{1}$ Rajaie Hospital, Pefusion, Tehran, Iran

${ }^{2}$ Rajaie Hospital, Heart Surgian, Tehran, Iran

${ }^{3}$ Rajaie Hospital, Anaesthesia, Tehran, Iran

\section{Abstract}

Backgrounds: Cardiopulmonary bypass ( $\mathrm{CPB}$ ) requirement in infants presents a unique challenge because of the large prime volume-to-blood volume ratio. Packed red blood cells (PRBCs) tend to deteriorate with long-term storage owing to their unphysiological composition and osmolality. Given that blood priming is inevitable in neonates, it is suggested that the metabolic load and osmolality be diminished before $\mathrm{CPB}$ initiation. We conducted the present study to test the hypothesis that the zero-balance ultrafiltration (Z-BUF) of the priming blood with $0.45 \%$ saline might be sufficient for modifying the metabolic load and osmolality and, thus, achieving a physiological state

Material and method: Sixty infants undergoing CPB were randomly assigned either to a control group or to a Z-BUF group and the Z-BUF of the priming blood was performed in the latter group. Electrolytes and osmolality were measured in the priming blood. The bleeding volume, the blood transfusion rate, the length of mechanical ventilation, the length of stay in the intensive care unit (ICU), the body temperature, and renal biomarkers were compared between the 2 groups.

Result: The osmolality and the levels of potassium, sodium, glucose, chloride, and lactate in the priming blood were significantly decreased after Z-BUF ( $\mathrm{P}<0.01)$. The Z-BUF group showed significant reductions in postoperative blood loss; postoperative blood transfusion; time to extubation; the length of stay in the ICU; the levels of lactate, sodium, and blood urea nitrogen at 24 hours postoperatively and the body temperature at 18 hours postoperatively $(\mathrm{P}<0.05)$. However, no statistically significant differences were found between the 2 groups regarding the body temperature and the levels of serum creatinine and blood urea nitrogen after admission to the ICU.

Conclusion: The results of the present study demonstrated that the Z-BUF of the priming blood could be a useful strategy in infants undergoing CPB insofar as it significantly modified the composition of the priming blood and improved the clinical outcome among our patients.

\section{Correlation of rewarming time} with arterial blood gas parameters in patient undergoing Coronary Artery Bypass Graft surgery

\author{
Rafiee S. ${ }^{1}$
}




\section{Abstract \\ Backgrounds: In patients under Coronary Artery Bypass Graft sur- gery patients with Cardio Pulmonary Bypass(CPB), , it is necessary to decrease the whole body temperature to protect vital organs. For weaning the patient from (CPB), it is important to rewarm the body Rewarming process may be influenced by such factors as hypother- mia temperature, rewarming time, CPB time, duration of surgery, an even the type of heat exchanger. Rewarming is a very important process that should be started during surgery and be finished at the end of CPB. Inappropriate rewarming may leads to complications as hypertension, increased $\mathrm{O} 2$ consumption, shivering, dangerous dysrhythmias(VF), increased production of $\mathrm{CO} 2$ and coagulopa- thies all of which are life-threatening for patient. \\ Material and method: This was a descriptive-correlationa study in which 83 patients under CABG surgery with CPB were recruited based on convenience sampling and inclusion criteria. The $\mathrm{ABG}$ results of seven times (before $\mathrm{CPB}$, before rewarming, at the start of rewarming in body temperature $32^{\circ} \mathrm{C}$, at the times the patient's temperature reach to 34 and $37^{\circ} \mathrm{C}$, before transfer to the intensive care unit(ICU), 5 to 15 minutes after the connec- tion to the ventilator in the ICU, and two hours after admission to ICU) were recorded and their relationship with the rewarm- ing time were examined. \\ Result: No statistically significant association was found be tween changes in arterial blood gases $(\mathrm{PaO} 2, \mathrm{PH}, \mathrm{PaCO} 2, \mathrm{HCO} 3)$ and rewarming time. Also, no significant correlation was found of the patientss arterial blood gas parameters with the duration of warm-up time. \\ Conclusion: It can be concluded that the arterial blood gas changes is not associated with rewarming time but with increas- ing rewarming time, the amount of lactate is increasing which has harmful metabolic effects. \\ The effect of different phases of menstrual cycle on respiratory indices in primary dysmenorrhea women}

Oraki F. ${ }^{1}$

${ }^{1}$ Physical Therapy, Physiotherapy, Ahvaz, Iran

\begin{abstract}
Backgrounds: Phphysiological changes during the menstrual cycle in primary dysmemorrhea women will modify the respiratory system, which can be a factor in reducing the pain tolerance threshold in these individuals. The aim of this study was to examine of menstrual cycle on respiratory indices in women with primary dysmemorrhea and their differences in different phases of menstrual cycle.

Material and method: This was conducted on women aged 18 to 25 years old with a painful menstruation a sample of 14 women suffered from primary dysmemorrhea. The respiratory parameters were measured by capnograph during the three phases of the bleeding menstrual cycle phase (day 3-4), follicular phase (day 10-11) and luteal phase (day 20-21).

Result: The results of this study showed a significant difference in end-tidal $\mathrm{CO} 2$ pressure in the follicular and luteal phase with bleeding phase ( $\mathrm{p}<0 \cdot 01)$, in addition respiratory rate and oxygen saturation showed a significant difference in different phases of menstrual cycle $(\mathrm{p}<0 \cdot 01)$.

Conclusion: The result of this study indicate that respiratory indices differ in different phases of menstrual cycle in women with
\end{abstract}

primary dysmemorrhea, which seems to be due to pain during bleeding phase in menstrual cycle and a factor in reducing the pain tolerance threshold. Therefore, it suggests using of respiratory rehabilitation for preventing of painful menstrual complications and subsequent absenteeism from work and school.

\section{A Review of Telerehabilitation Methods for Monitoring Cardiovascular and Pulmonary Patients outside the Rehabilitation Centers}

\author{
Boozari ${ }^{1}{ }^{1}$ \\ ${ }^{1}$ Faculty Of Medical Sciences Tarbiat Modares University, Assistant Professor Depart- \\ ment Of Physiotherapy, Tehran, Iran
}

\section{Abstract}

Backgrounds: In telerehabilitation, patients will be monitored outside the rehabilitation centers. With the use of portable devices or applications, the therapeutic team collect the necessary data to assess and prescribe the required therapeutic programs for each patient. Telerehabilitation is used in different fields of medicine. However, its usage for cardiovascular/pulmonary patients is more vital, because these patients need an appropriate and longtime rehabilitation program for reducing the risk of mortality in them. Therefore, we aim to review the articles related to telerehabilitation.

Material and method: Articles with different keywords related to telerehabilitation and cardiovascular/pulmonary diseases have been studied.

Result: In telerehabilitation, accelerometers or accelerometers embedded in the mobile phones attach to the patients' body and collect the kinematic data. Using this data, various performance parameters from patients' movements can be measured. Therefore, patients' performance can be monitored in their homes or workplaces and in more functional situations. Studies showed that the patients follow the rehabilitation protocols more thoroughly in the telerehabilitation programs. Moreover, the patients' life style can change using the telerehabilitation methods.

Conclusion: With the advancement of science, the devices that can assess the performance became smaller, cheaper and easier to use for both patients and clinicians. These devices can be used to assess the way the patients move, behave and perform the therapeutic protocols outside the clinics which is more useful for patients needed a longtime rehabilitation care. However, these devices and applications are not common in Iran which needs more attention from both clinicians and biomechanists.

\section{Effects of resistance training on cardiovascular parameters and glycemic control in patients with type 2 diabetes}

\author{
Saeidi M. ${ }^{1}$, Ravanbod R. ${ }^{1}$ \\ ${ }^{1}$ Tarbiat Modares University, Physiotherapy, Tehran, Iran
}

\section{Abstract}

Backgrounds: Exercise performs a primary role in prevention 
and control of type 2 diabetes (T2D). Although the positive effects of aerobic exercise in T2D patients have been addressed, resistance training (RT) has been debated because of acute stress and overloading on vascular system, challenged by hyperglycemic conditions. This study summarized the effects of RT on cardiovascular parameters and glycemic control in patients with T2D.

Material and method: A systematic literature search was carried out in PubMed, google scholar, science direct databases for studies published in English (1998 to 2018) combining the terms resistance training, type 2 diabetes, strength, and cardiovascular function.

Result: We included 19 studies with T2DM in our study. RT makes improvements in body composition (via an increase in lean body mass which uses glucose from the blood stream to produce energy), insulin resistance and glycemic control, with a reduction of glycosylated hemoglobin (HbA1c), increase in muscle mass, decrease fat mass, visceral fat and inflammatory markers, improvements lipid profiles, blood pressure, cardiac performance, strength and muscular power, mitochondrial content of the skeletal muscle, bone mineral density, daily energy expenditure and quality of life as well as excess oxygen delivery post-training and heart rate variability. Studies indicate that exercise training positively influence different aspects of diastolic function, however, the influence of type of exercise remains unclear.

Conclusion: The effects of RT on metabolic control, and cardiovascular dysfunction, guarantee the practice of metabolic rehabilitation for autonomic modulation in a safety way, to improve quality of life and reduce morbidity and mortality rates related to CVD in $\mathrm{T} 2 \mathrm{D}$.

\section{Reaction Time and Anticipatory Skill in Patients Undergoing Percutaneous Coronary Intervention Compared with Normal Control A Pilot Study}

\author{
Dabbaghipour N. ${ }^{1}$, Sadeghian M. ${ }^{2}$, Attarbashi \\ Moghadam B. ${ }^{1}$, Shadmehr A. ${ }^{2}$ \\ ${ }^{1}$ Tehran University Of Medical Science, Physiotherapy, Tehran, Iran \\ ${ }^{2}$ Tehran University Of Medical Science, Medicine, Tehran, Iran
}

\section{Abstract}

Backgrounds: Percutaneous coronary intervention (PCI) is a common invasive procedure for the treatment of coronary artery diseases (CAD). One of the most common disorders associated with these procedure is occurrence of cognitive impairments (CIs).

The aim of this study was to assess reaction time and anticipatory skill of PCI patients and normal people with using a computer based software program as a safe and easy method.

Material and method: 10 male PCI patients and 10 male healthy individuals participated in this cross sectional study. Visual choice reaction time, visual complex choice reaction time, auditory choice reaction time, auditory complex choice reaction time and anticipatory skill of the high and low speed of the ball of both groups were analyzed by Speed Anticipation Reaction Test (SART) software.

Result: No significant difference was seen between two groups in terms of complex visual and auditory reaction time tests and anticipation skill with low speed ( $>>0.05)$. However, PCI patients had a slower simple visual choice reaction time and lower anticipatory skill of the high speed of the ball tests compared normal group.

Conclusion: Assessment of reaction time and anticipatory skill such as a neurocognitive tests are considered to be one of the useful methods for evaluating the cognition function of patients who have cardiovascular disease. Furthermore, the pilot study suggest that PCI patients had a similar or even poorer performance in cognitive function compared with healthy people.

\section{- Effect Of Exercise On Hypertension}

\section{Khosravian Arab T. ${ }^{1}$ \\ ${ }^{1}$ Tehran University Of Medical Science, Faculty of Physiotherapy, Tehran, Iran}

\section{Abstract}

Backgrounds: High BP is a primary risk factor for stroke, congestive heart failure, angina, renal failure, and myocardial infarction at all ages and in both genders. Hypertension clusters with hyperlipidemia, diabetes, and obesity, each intensifying the risk of hypertension. Because of the health risks of hypertension, prevention and treatment including exercise is needed to improve patient outcomes.

Classification of Blood Pressure

Blood Pressure Category Systolic (mm Hg) Diastolic (mm Hg)

- $\quad$ Optimal $<120<80$

- $\quad$ Normal $<130<85$

- $\quad$ High Normal 130-139 85-89

- Hypertension

- $\quad$ Stage 1(Mild) 140-159 90-99

- $\quad$ Stage 2 (Moderate) 160-179 100-109

- $\quad$ Stage 3(Severe) $\square 180 \square 110$

Material and method: Possible Mechanisms of BP Reduction with Exercise Training

1. Lower cardiac output and peripheral vascular resistance at rest and at any given sub maximal level of work

2. Reduction in blood catecholamine levels and plasma renin activity

3. Reduction in central fat independent of changes in body weight or body mass index

4.Altered renal function to increase elimination of sodium leading to reduce fluid volume

Result: Exercise Guidelines

1. No special guidelines for mild to moderate hypertension

2. Endurance training for mild hypertension ,3-5 days/week ,2060 minutes, $50-85 \%$ of maximal oxygen uptake

3. Lower intensities may be required until BP control is achieved although no specific guidelines exist

Resistive Training

Resistive exercise produces the most striking increases in BP .Because resistive exercise also results in less of a HR increase compared with aerobic exercise the total myocardial burden may be less than aerobic exercise . Combined training (weight and aerobic exercise) has been shown to reduce both SBP and DBP by as much as $13 \mathrm{~mm} \mathrm{Hg}$.

Conclusion: Hypertension is a major risk factor for CVD. Exercise is a key component in the prevention and treatment of hy- 
pertension. Exercise training can be expected to reduce SBP and DBP by 7-10 mm Hg.

\section{Effect of Cardiac Rehabilitation on Lipid Profiles in Patients with Cardiovascular Disease A Systematic Review}

\author{
Javaherian M. ${ }^{1}$, Dabbaghipour N. ${ }^{1}$ \\ ${ }^{1}$ Tehran University Of Medical Science, Physiotherapy, Tehran, Iran
}

\begin{abstract}
Backgrounds: Cardiac rehabilitation (CR) as a secondary preventive measure can reduce the mortality and prevent progression of disorder in cardiovascular disease (CVD). Therefore, the present systematic review was conducted to assess the effect of CR program on lipid profile (LP) in patients with CVD.

Material and method: PubMed, SCOPUS and Web of science databases were searched from January 1, 1980, to November 30, 2017 with no language restriction. Initially, all published randomized controlled trials which had reported the efficacy of CR in patients with CVD or patients undergoing cardiac surgery that measured any type of LP were included by 3 independent authors. All studies were critically appraised and assessed their quality based on PEDro scale by two independent authors.

Result: Initially 681 published article reviewed that 7 of them included to study finally. Most studies showed improvement of in TC and TG after CR in both surgical \& non-surgical subgroup significantly. However, the rehabilitation program did not show any significant difference of HDL amount $(\mathrm{P}=0.761)$ and LDL $(\mathrm{P}=0.047)$ level after $\mathrm{CR}$

Conclusion: It seems CR can improve LP in patients with CVD which can be considered as a program for risk factor reduction in patients with any type of CVD. Further high-quality studies with more sample size are needed to assess CR in surgical patients except coronary artery bypass surgery and patients with myocardial infraction.

Keyword: cardiac rehabilitation, cardiovascular disease, lipid profile.
\end{abstract}

\section{- Effect of aerobic and resistance exercises on cardiovascular fitness functional ability and muscle strength in children with hemophilia}

\author{
Marzban V. ${ }^{1}$ \\ ${ }^{1}$ Iranian Comrehensive Hemophilia Care Center, Physiotherapy, Tehran, Iran
}

\begin{abstract}
Backgrounds: Children with hemophilia are at risk for reduced cardiovascular fitness, muscle strength and functional ability as a result of reduced physical activity and less involvement in intense activities. So, the purpose of this study was to investigate the effect of resistance and aerobic exercise program on cardiovascular fitness, muscle strength and functional ability in children with hemophilia.
\end{abstract}

Material and method: 20 boys with hemophilia A and B ranging in age from 9 to 13 years had participated in this study. They were assigned randomly into two equal groups (control and study). Control group received a designed physical therapy program and aerobic exercise in the form of Elliptical machine, while the study group received the same program as the control group in addition to resistance training program in the form of bicycle ergometer training and weight resistance. Both groups received treatment sessions three times per week for 3 months .strength of knee muscles and functional ability were evaluated before and after the 3 months of treatment program.

Result: Significant improvement was observed in cardiovascular fitness, knee extensors and flexors strength, and functional ability in the study group when comparing pre and post treatment measurements. There was a significant improvement in functional ability of the control group. Significant difference was also observed between both groups when comparing the post treatment measurements in favor of the study group.

Conclusion: Based on obtained data, resistance and aerobic exercise training program is effective in increasing cardiovascular fitness, muscle strength and functional ability in children with hemophilia

\section{Effect of Cognitive Behavior Therapy on cardiac rehabilitation Review article}

\section{Rahimi F. ${ }^{1}$, Arzani P. ${ }^{2}$} ${ }^{1}$ Musculoskeletal Rehabilitation Research Center Rehabilitation School Jundishapur
University Of Medical Sciences, Physiotherapy Department, Ahvaz, Iran
${ }^{2}$ Rehabilitation School Of Shahid Beheshti University Of Medical Sciences, Physio-
therapy Department, Tehran, Iran

\section{Abstract}

Backgrounds: Factors such as low socioeconomic status, social support/isolation, depression, stress and distress, personality and sleep disturbance increase risk of cardiovascular events and negatively affect on quality of life. These factors may have direct impacts on cardiovascular disease through immune or neuroendocrine pathways, or more indirect impacts, by limiting adherence to recommended therapies and cardiac rehabilitation. CVD patients experience a more rapid loss of physical function and onset of physical disability, greater symptom severity, poorer health-related quality of life (HRQOL), more frequent hospitalizations, and higher mortality rates. The aim of this study was to evaluate the efficacy of cognitive behavior therapy in these patients.

Material and method: Published studies from 2000-2018 were searched on the Google Scholar, Medlib, PubMed, Science Direct and Scopus databases. These keywords were used: Cognitive behavior therapy, cardiac rehabilitation, cardiovascular disease, myocardial infarction, heart failure and hypertension. 125 studies were found that finally according to the keywords and inclusion criteria 10 randomised control trial studies were included. Result: CBT strategies promote a positive health attitude, and facilitate adaptation, reducing the psychological burden. CBT may be effective in reducing depression severity and better physical function and HRQOL.

Conclusion: To sum up CBT is an effective approach when combined with standard care. 


\section{- The Investigation of Barriers to Participation in Cardiac Rehabilitation and How to Overcome}

\author{
Zafarnejad M. ${ }^{1}$ \\ ${ }^{1}$ Day Hospital, Physical Therapy, Tehran, Iran
}

\begin{abstract}
Backgrounds: Cardiac rehabilitation (CR) which is strongly
\end{abstract} recommended in international guidelines, is a complex intervention offered to patients diagnosed with heart disease, and consists of physical activity, health education, advice on cardiovascular risk reduction, and stress management. Cardiac Rehabilitation also improves exercise capacity, quality of life and psychological well-being. The evidence that cardiac rehabilitation reduces mortality, morbidity and unplanned hospital admissions, is increasing. Regrettably, despite international recommendations, and despite the proven clinical and economical benefits of $\mathrm{CR}$, as the only proven model that significantly reduces the mortality and morbidity, referral to and participation rates in CR are low and cardiac rehabilitation remains a chronically-underutilized resource. This review focuses on what cardiac rehabilitation is, the barriers to cardiac rehabilitation participation and how to overcome these barriers.

Material and method: Globally, the prevalence of coronary heart disease and heart failure is increasing. Also with improved survival and an aging population, the number of people living with coronary heart disease has increased to an estimated 2.3 million. In the United States It is estimated that approximately 600,000 people die each year from CVD-related causes, equivalent to three every one minutes, and in the UK, around 175,000 have an acute myocardial infarction every year, equivalent to one every three minutes.

Unfortunately effective implementation of cardiac rehabilitation after acute coronary syndrome, coronary revascularisation, and heart failure has remained suboptimal with overall participation rates $<50 \%$. Cardiac rehabilitation and secondary prevention services are comprehensive and long term programmes involving medical evaluation, prescribed exercise, cardiac risk factor modification, education, and counselling.These programmes are designed to limit the physiological and psychological effects of cardiac illness, reduce the risk for sudden death or re-infarction, control cardiac symptoms, stabilize or reverse the atherosclerotic process, and enhance the psychosocial and vocational status of selected patients. The other components of comprehensive rehabilitation programmes includes to optimise cardiovascular risk reduction, develop healthy behaviours, reduce disability, and promote an active lifestyle.

The latest updated Cochrane review of exercise based cardiac rehabilitation reports:

-An absolute risk reduction in cardiovascular mortality from $10.4 \%$ to $7.6 \%$,

-Risk reduction of hospital admission from $30.7 \%$ to $26.1 \%$ for patients after myocardial infarction and revascularization,

-Decrease in symptoms of depression from $22 \%$ to $13 \%$, also depressed patients who completed their cardiac rehabilitation had a $59 \%$ lower mortality from $44 \%$ to $18 \%$.

The latest studies about the risk of cardiac rehabilitation which is reported in US, shows one case of ventricular fibrillation per 111,996 patient-hours of exercise and one myocardial infarction per 294,118 patient-hours.
Result: Poor uptake of cardiac rehabilitation and the barriers to CR participation has been attributed to several factors:

1) Poor referral rates, especially for certain groups such as Women, People from ethnic minority groups, Elderly people, People living in rural settings and People in low socioeconomic classes. 2) Lack of endorsement by a doctor.3) Multiple morbidities, leading to poor functional capacity. 4-Depression. 5) Obesity (high body mass index). 6) Poor patient adherence which leading to low enrolment and high dropout rates. 7) Cigarette smoking. 8) Poor exercise habits.

9- Problems with transport. 10-Poor social support. 11- Lack of leave from work to attend center-based sessions.

The most effective way to increase uptake and optimise adherence to cardiac rehabilitation is: 1.Improving CR Referral, by using systematic referral to outpatient CR during the hospital discharge process. 2. Improving CR Enrollment, The Systematic approaches to CR enrollment substantially increase CR participation rates. 3. Increasing Adherence to CR, ensuring that all patients receive the largest "dose" possible of program participation.

Conclusion: The benefits of CR are broad and compelling, ranging from decreased mortality and decreased hospitalizations to improvements in functional capacity, depression, and quality of life. It will be achievable through individual and collective action to implement evidence-based strategies that increase CR referral, enrollment, and adherence. A concordant effort from hospitals and CR programs to increase capacity will also be needed. Doing so will save lives, reduce avoidable hospitalizations, and improve the quality of life for cardiovascular patients.

Keywords: Cardiac rehabilitation, Barriers, Overcoming barriers

\section{Exercise training progression in cardiac rehabilitation}

\author{
Fasihnia S. ${ }^{1}$, Mosallanezhad Z. ${ }^{1}$, Abdoos M. ${ }^{1}$ \\ ${ }^{1}$ University Of Social Welfare And Rehabilitation Sciences, Physical Therapy, Tehran, \\ Iran
}

\section{$\underline{\underline{\text { Abstract }}}$}

Backgrounds: Exercise training is a prominent and critical component of early outpatient cardiac rehabilitation (CR), providing impressive patient benefits for cardiorespiratory and metabolic indices, quality of life, and cardiovascular disease management.

Material and method: Aerobic and resistance exercise training is a cornerstone of early outpatient cardiac rehabilitation (CR) and provides impressive benefits for patients. The exercise dose is commonly defined as the total amount of energy expended during exercise training over a period of $1 \mathrm{wk}$. For aerobic exercise training, it is the combination of frequency (sessions per wk), intensity (how hard), type (modality), and time (total duration).for aerobic exercise training kilocalories (kcal) expended per wk and metabolic equivalents per min (MET-min) of exercise are used. Overload is defined as an exercise dose which is above and beyond the accustomed amount of exercise for a given individual. For aerobic exercise training, overload may be accomplished by increasing any 1 of the components of the exercise dose and For resistance exercise training, either increasing the frequency of sessions, the amount of resistance, the number of repetitions per set, the number of sets per exercise or decreasing the rest period between sets provides overload. 
Conclusion: The most challenging and least well-defined aspect of the exercise prescription for patients in $\mathrm{CR}$ is the progression of the dose of exercise.

\section{The role of cytokines in heart disease focus on interleukin 6}

\author{
Rahmati Yami M. ${ }^{1}$, Ravanbod R. ${ }^{1}$ \\ ${ }^{1}$ Tarbiat Modares University, Physiotherapy, Tehran, Iran
}

\begin{abstract}
Backgrounds: Cytokines are a broad category of small proteins, that are an important role in cell signaling.

Can be divided to Anti-inflammatory and pro-inflammatory cytokines.

The purpose of this study was to investigate the effects of cytokines on the patient with heart disease

Material and method: Search in PubMed and Elsevier database from the earliest available date to October 2018, using various combinations of keywords such as "heart"," cytokines", interleukin", "IL". from 207 articles, 16 studies include this narrative review

Result: IL-6 is an interleukin that acts as both a pro-inflammatory cytokine and an anti-inflammatory myokine

Exercise Decreases in total cholesterol and triglycerides leve and Decreased pro-inflammatory cytokines such as Tumor-necrosis factor- $\alpha$, Interleukin- $1 \beta$, Interleukin- 6 .

Conclusion: pro-inflammatory cytokines could be used markers for risk stratification and prognostication. some reports have sug gested that inflammatory cytokines may predict adverse outcome.
\end{abstract}

\section{ICF-Based Approach in Cardiac Rehabilitation}

\section{Saba M. ${ }^{1}$}

${ }^{1}$ Musculoskeletal Rehabilitation Research Center Ahvaz Jundishapur University of Medical Sciences Ahvaz Iran, Physical Therapy, Ahvaz, Iran

\begin{abstract}
Backgrounds: As an International Standard to define Health and Function, International Classification of Functioning, Disability and Health (ICF) that proposed by the WHO, is a new classification system to introduce health component based on interaction patterns. The "function", "health", and "disability" are independent and correlated. This tool quantify disability as an impairment at body, personal or social level that a patient may experience during interaction with contextual factors. For being applicable in clinical practice, different ICF core sets are published for musculoskeletal, neurological and cardiovascular conditions, among them are core sets such as Diabetes Mellitus, Obesity and ICF Core Set for Chronic Ischemic Heart Disease (CIHD). Cardiac Rehabilitation is proved to be as a secondary prevention and is recommended in cardiology society guidelines. Material and method: There was a formal conference to establish the Comprehensive and Brief ICF Core Sets for patients with CIHD. Sixteen experts (physicians in various sub-specialties, physiotherapists, epidemiologists) from 7 different countries decided which ICF categories are to be included in the ICF Core Sets for CIHD following a formal, decision-making and consen-
\end{abstract}

sus process. It is a standard tool for gathering, scaling and analyzing detailed data consistent with disability and function. According to ICF core sets for CIHD, classification of component "activities and participation" consists; carrying out daily routine, handling stress and other psychological demands, lifting and carrying objects, walking, doing housework, driving, etc.

Result: The importance of evaluating symptoms and functional limitations for managing CIHD leads to development and use of some measures related to condition-specific health status. The 61 categories of the Comprehensive ICF Core Set are made up of $14(23 \%)$ categories from the component body functions, one (2\%) category from the component body structures,17 (28\%) categories from the component activities and participation and 29 (48\%) categories from the component environmental factors. From 61 classifications in The Comprehensive ICF Core Set categories, 36 ICF categories were chosen to be as The Brief ICF Core Set, short adequately to be applicable in clinical practice. Conclusion: For providing clinically relevant data throughout the healthcare process from the acute setting to outpatient management in cardiac rehabilitation, the ICF seems to be a suitable tool to allow practitioners collecting data related to disability and functioning.

\section{- Implementation of An ECG Holter Monitor Recorder and Analyzer Software, and its Clinical Trial}

Shirafkan R. ${ }^{1}$, Ghanbari M. ${ }^{1}$, Moftakharzadeh A. ${ }^{1}$, Shoaei O. ${ }^{1}$, Ghadrdoost B. ${ }^{2}$, and Fazelifar A. F. ${ }^{2}$

'Niktek Fanavari, Tehran, Iran. (E-mail: info@niktek.ir).

${ }^{2}$ Rajaie cardiovascular medical and research center, Iran University ofmedical science, Tehran, Iran

\section{Abstract}

An ECG Holter monitor is a portable device that records ECG signals continuously. Then, the records are transferred to the computer for further analysis in a specially designed software. Making use of the software, the technician prepares a report for the doctor in order to diagnose probable arrhythmias. NTH48i is the first Iranian ECG Holter monitor that is compliant with the IEC60601-2-47 (Edition 2.0 2012) standard. The device uses a rechargeable battery as a power source. Utilizing the ultra-low power design which is presented in this presentation, the device can record ECG signal up to 14 days continuously. The software designed for the analysis of the ECG signal uses an effective R-detection algorithm with $99.8 \%$ precision. The presented algorithm is applied on MIT and AHA databases in order to verify the effectiveness of the NT Cardio-Analyzer software. The hardware and software are verified through a clinical test in the Rajaie Hospital. The device was successfully tested on 60 patients and the results were compared to those of the commercial Meditech CardioMera holter monitor used as reference.

\section{An Ultra-Low-Power IC for Implantable Cardiac Pacemakers and/ or Ambulatory ECG Recorders}

\author{
Rezaeiyan Y. ${ }^{1}$, Zamani M. ${ }^{1}$, Shoaei O. ${ }^{1}$, and \\ Serdijn W. A.
}


${ }^{1}$ Bio-Integrated Systems Lab, School of Electrical and Computer Engineering, University of Tehran, Tehran, Iran. (E-mail: oshoaei@ut.ac.ir).

${ }^{2}$ Bio-Electronics, Faculty of Electrical Engineering, Mathematics and Computer Science, Delft University of Technology, 2628 CD Delft, The Netherlands.

\section{Abstract}

In this project, a newly designed and fabricated ultra low power mixed-signal IC (Integrated Circuit) for implantable pacemakers is presented. The proposed system consists of four major building blocks: 1) a sense amplifier, comprising three readout channels for acquiring IECG signals, 2) phase and amplitude bioimpedance readout channels for extracting intra cardiac bioimpedance, 3 ) a high voltage output pulse generator to stimulate the heart muscle, and 4) a transmitter for transmitting the sense channel and the bio-impedance channel outputs.

The proposed low power mixed signal IC is fabricated in a 0.18 $\mu \mathrm{m}$ HV CMOS process and occupies $2.38 \mathrm{~mm} 2$ (Fig. 1). The biopotential channels extract the heart signals with 9.2 effective number of bits (ENOB) and the bioimpedance channels measure the amplitude and phase of the heart impedance with $1.35 \Omega \mathrm{rms}$ accuracy. The complete IC consumes only $4.2 \mu \mathrm{A}$ from a $1 \mathrm{~V}$ power supply. The high-voltage monophasic stimulator output with 15 different amplitudes can also be programmed from $1 \mathrm{~V}$ to $7.8 \mathrm{~V}$.

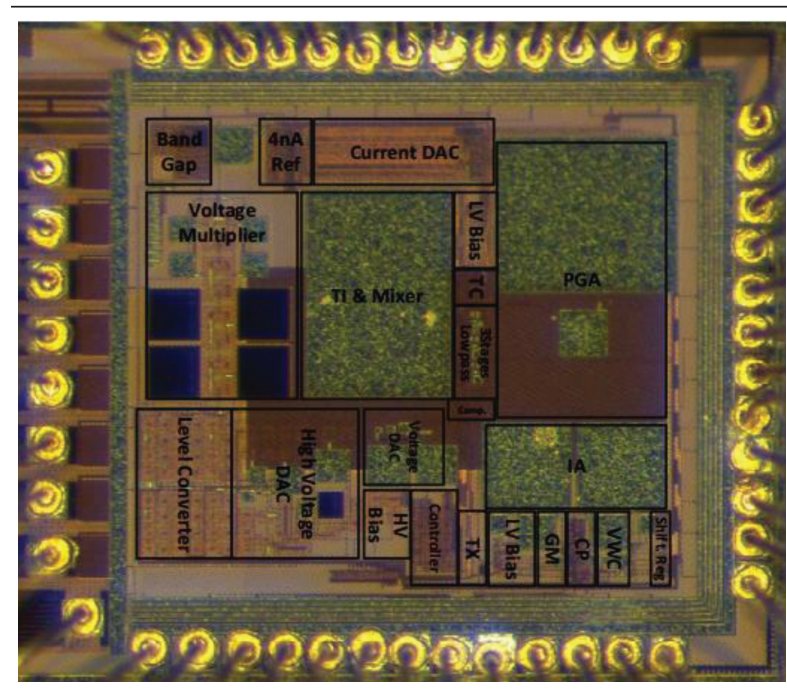

Fig 1. Chip micrograph

\section{Burden of Structural Heart Diseases in School-Aged Population: Protocol of SHED LIGHT Study (Structural HEart Diseases in pupiLs by echocardIoGrapHic Test)}

Samiei N. ${ }^{1}$, Noohi F. ${ }^{2}$, Maleki M. ${ }^{2}$, Tabib A. ${ }^{3}$, Parsaee M. ${ }^{4}$, Shojaei Fard M. ${ }^{4}$, Emkanjoo Z. ${ }^{5}$, Peighambari M. M. ${ }^{1}$, Mohebbi A. ${ }^{1}$, Omrani Gh. ${ }^{1}$, Bakhshandeh H. ${ }^{1}$, Amin A. ${ }^{3}$, Haghjoo M. ${ }^{5}$, Saedi S. ${ }^{3}$, Alizadeh Ghavidel A. ${ }^{1}$, Naderi N. ${ }^{3}$, Taghavi S. ${ }^{3}$, Nikpajooh A. ${ }^{3}$, Moradian M. ${ }^{3}$, Rahsidi Ghader F. ${ }^{3}$, Rafi Khorgami M. ${ }^{3}$, Khajali Z. ${ }^{3}$, Rezaei Y., Hosseini S. ${ }^{1}$
${ }^{1}$ Heart Valve Disease Research Center, Rajaie Cardiovascular Medical and Research Center, Iran University of Medical Sciences, Tehran, Iran

${ }^{2}$ Cardiovascular Intervention Research Center, Rajaie Cardiovascular Medical and Research Center, Iran University of Medical Sciences, Tehran, Iran

${ }^{3}$ Rajaie Cardiovascular Medical and Research Center, Iran University of Medical Sciences, Tehran, Iran

${ }^{4}$ Echocardiography Research Center, Rajaie Cardiovascular Medical and Research Center, Iran University of Medical Sciences, Tehran, Iran

${ }^{5}$ Electrophysiology Research Center, Rajaie Cardiovascular Medical and Research Center, Iran University of Medical Sciences, Tehran, Iran

\section{Abstract}

The structural heart diseases (SHD) mainly include valvular heart disease (VHD) and congenital heart diseases (CHD). The SHDs have great impacts on health care systems with growing public health problems, and can be associated with high rates of morbidity and mortality if left untreated. There is lack of proper data demonstrating the exact prevalence of people living with $\mathrm{CHD}$, even in developed countries. In a populationbased study, we will evaluate the prevalence of structural heart diseases (SHDs) in school-aged individuals. We randomly selected six districts of Tehran urban area; then, we identify 46 schools for the evaluation of students using a two-stage cluster sampling method. The sample size from each region was proportionate to the population of either district. The final sample size of main study will be $\approx 15,000$ students. The number of schools for both genders and age groups will be similar to have a relatively equal number of both sexes and age groups in our final cohort. After school selection, the parents of all students in the selected institution were provided consent form. The members of school management department will inform regarding the details of study and they transferred required information to all parents via telephone or close interviews. The students whose parents singed consent form will be examined. All steps of examinations will be carried out on-site in a standard room Examinations will include vital sing measurements, electrocardiogram evaluation, and echocardiographic assessments. All echocardiographic examinations will be performed by experienced cardiologists/echocardiographers and the measurement of vital signs and electrocardiogram will be done by educated and experienced nurses. All echocardiographic examinations will be performed using pocket-sized ultrasound device. The SHED LIGHT study is now running in the schools of Tehran urban area.

\section{How is it possible to increase the safety in cardiac surgery "Twenty years experiences"}

\author{
Masoumi M. ${ }^{1}$, Dehghani E. ${ }^{1}$, Chehri G. ${ }^{1}$ \\ ${ }^{1}$ Bistoon hospital Iran, kermanshah
}

\section{Abstract}

Backgrounds: Increasing the safety in cardiac surgery is the key point of success. Surgical bleeding is one of the catastrophic complication, specially in cases of aortic root surgeries. lack of safety in other parts of the heart, may result sever complications. Such as paravalvular leakage In mitral or aortic valve replacement(MVR), post operative bleeding In Bental operation and even residual ASD ,in repair of primary ASD

Material and method: We used some innovations in many 
cardiac surgeries during last twenty years with good long-term results. for prevention of bleeding in suture lines and paravalvular leakage in mitral valve replacement, proximal anastomosis, both coronary reimplantation and distal anastomosis during Bental operation both we used stripes of pericardium as a necklace.

Result: The rate of surgical bleeding, paravalvular leakage in MVR and residual ASD were significantly reduced

Conclusion: We called these necklaces as "golden collar", because of golden effect of this method which is obvious during the surgery. These necklaces may be from pericardium, Dacron or suture material In other operations such as CABG in diffuse disease of the coronary arteries, prevention of aortic dilatation in mild to moderate aortic dilatation and other routine operations, we describe our experiences for increasing the safety of operations

\section{Efficacy of Dexmedetomidine in Coronary Artery Bypass Graft Surgery under Cardiopulmonary Bypass}

Sedighinejad A. ${ }^{1}$, Mohammadzadeh Jouryabi A. ${ }^{1}$, Vali Imantalab ${ }^{1}$, Mirmansouri A. ${ }^{1}$, Nassiri Sheikhani N. ${ }^{1}$, Atrkarroushan Z. ${ }^{2}$, Biazar G. ${ }^{1}$, Chaibakhsh Y. ${ }^{2}$

Anesthesiology Research Center Iran, rasht

${ }^{2}$ Guilan University of Medical Sciences Iran, rasht

\section{Abstract}

Backgrounds: In patients undergoing Cardiopulmonary Bypass $(\mathrm{CPB})$ with extracorporeal circulation, the rapid restoration of blood flow to the ischemic tissue induces cardiac damage termed as myocardial Ischemic Reperfusion (I/R) injury.

Material and method : Material: We hypothesized that Dexmedetomidine modulates Ischemic/Reperfusion injury in Coronary Artery Bypass Graft Surgery (CABG) under Cardiopulmonary Bypass.

Methods: This randomized double-blind trial took place at Dr. Heshmat Hospital affiliated to Guilan University of Medical Sciences. From April 2016 to March 2017, eligible CABG patients were randomized to receive either Dexmedetomidine infusion 0.3 to $0.5 \mathrm{mcg} / \mathrm{kg} /$ hour before induction of anesthesia till 12 hours postoperatively (group D) or normal saline as placebo (group P). Creatinine phosphokinase-MB(CKMB) and cardiac troponin I (cTnI) levels were measured at baseline (To) ,6, 12, 24, and 48 hours after the operation (To-T4).

Result: 114 patients 58 in group Dex and 56 in group P were analyzed. No significant differences were found between 2 groups, in view of baseline characteristics. Following CPB, a marked increase in CKMB and cTnI plasma levels was observed compared with baseline $(P=0.0001)$. Serum CKMB levels increased from $2.27 \pm 0.59$ to $7.81 \pm 1.39$, and $2.22 \pm 0.64$ to $7.46 \pm 1.25$ and cTnI levels from $10.22 \pm 0.17$ to $4.89 \pm 1.1$, and $0.27 \pm 0.28$ to $4.5 \pm 1.4$ in groups $\mathrm{C}$ and $\mathrm{D}$, respectively ( $\mathrm{P}=0.0001)$. According to $\mathrm{CKMB}$, there was a significant difference between the two groups at T2 $(\mathrm{P}=0.002)$ and T3 $(\mathrm{P}=0.0001)$, and based on $\mathrm{cTnI}$ at T2 $(\mathrm{P}=$ $0.004)$ and T3 $(P=0.0001)$. No adverse effect was recorded due to this intervention.

Conclusion:Perioperative Dexmedetomidine in cardiac surgery appears safe, with properties to alleviate Ischemic/Reperfusion injury.
- Effects of resistance training on cardiovascular parameters and glycemic control in patients with type 2 diabetes

\author{
Saeidi M. ${ }^{1}$, Ravanbod R. ${ }^{1}$ \\ ${ }^{1}$ Tarbiat Modares University Iran, Tehran
}

\section{Abstract}

Backgrounds: Exercise performs a primary role in prevention and control of type 2 diabetes (T2D). Although the positive effects of aerobic exercise in T2D patients have been addressed, resistance training (RT) has been debated because of acute stress and overloading on vascular system, challenged by hyperglycemic conditions. This study summarized the effects of RT on cardiovascular parameters and glycemic control in patients with T2D.

Material and method: A systematic literature search was carried out in PubMed, google scholar, science direct databases for studies published in English (1998 to 2018) combining the terms resistance training, type 2 diabetes, strength, and cardiovascular function.

Result: We included 19 studies with T2DM in our study. RT makes improvements in body composition (via an increase in lean body mass which uses glucose from the blood stream to produce energy), insulin resistance and glycemic control, with a reduction of glycosylated hemoglobin (HbA1c), increase in muscle mass, decrease fat mass, visceral fat and inflammatory markers, improvements lipid profiles, blood pressure, cardiac performance, strength and muscular power, mitochondrial content of the skeletal muscle, bone mineral density, daily energy expenditure and quality of life as well as excess oxygen delivery post-training and heart rate variability. Studies indicate that exercise training positively influence different aspects of diastolic function, however, the influence of type of exercise remains unclear.

Conclusion: The effects of RT on metabolic control, and cardiovascular dysfunction, guarantee the practice of metabolic rehabilitation for autonomic modulation in a safety way, to improve quality of life and reduce morbidity and mortality rates related to CVD in T2D.

\section{Major cardiovascular risk factors patterns during 13 years follow-up among Iranian adults Isfahan Cohort Study}

\author{
Heshmat-Ghahdarijani K. ${ }^{1}$, Sadeghi M. ${ }^{1}$, \\ Sarrafzadegan N. ${ }^{1}$, Dianatkhah M. ${ }^{1}$, \\ Vakhshoori M. ${ }^{1}$, Roohafza H. ${ }^{1}$
}

${ }^{1}$ Isfahan University of Medical Sciences Isfahan Iran Iran, Isfahan

\section{Abstract}

Backgrounds: Objectives: major cardiovascular risk factors trends have been evaluated less frequently in Middle-East nations. The aim of study was assessing those factors among Iranian persons. 
Material and method: 6504 participants aged at least 35 years were recruited for 12 years of longitudinal population based study started in 2001 till 2013 in three phases. Systolic blood pressure (SBP), diastolic blood pressure (DBP), waist circumference (WC), waist to hip ratio (WHR), body mass index (BMI), fasting blood sugar (FBS) and lipid indices including total cholesterol (TC), triglyceride (TG), low-density lipoprotein cholesterol (LDLC), high-density lipoprotein cholesterol (HDL-C) and TG to HDL-C ratio (TG/HDL-C) were considered risk factors and measured in 2001, 2007 and 2013.

Result: during the follow-up duration, mean ranges of SBP (119.3 \pm 18.7 to128.4 $\pm 17.5 \mathrm{mmHg}, \quad \mathrm{P}<0.001)$, DBP $(76.7 \pm 11.8$ to82.8 $\pm 12.5 \mathrm{mmHg}, \mathrm{P}<0.001)$, BMI $(27.4 \pm 4.4$ to $28 \pm 4.6 \mathrm{~kg} / \mathrm{m} 2$, P $<0.001)$ and FBS $(86.7 \pm 29.6$ to106.5 $\pm 38 \mathrm{mg} / \mathrm{dl}, \mathrm{P}<0.001)$ were increased in both sexes. Favorable downward patterns were observed in terms of TC $(219.5 \pm 51.3$ to200.1 $\pm 40.9 \mathrm{mg} / \mathrm{dl}, \mathrm{P}<0.001)$, TG $(200.2 \pm 110.3$ to155.1 $\pm 85 \mathrm{mg} / \mathrm{dl}, \mathrm{P}<0.001)$, LDL-C (132.6 \pm 42.4 to111.7 $\pm 27.8 \mathrm{mg} / \mathrm{dl}, \mathrm{P}<0.001)$ and TG/ HDL-C $(4.5 \pm 2.8$ to3.9 $\pm 2.8, \mathrm{P}$ $<0.001)$, but HDL-C levels showed less desirable result. WC and WHR patterns revealed controversial results in each gender, but in total population those variabilities were not significant. Conclusion: in spite of desirable trends observed in most lipid indices, other cardiovascular risk factors showed either insignificant or inverse relation. Multiple health strategies required in order to decline the trends of those risk factors showing unfavorable trends.

\section{Comparative study of cardiac} muscle damage markers and clinical outcomes in patients undergoing pulmonary valvular surgery using a cardiopulmonary bypass device using two Beating Heart pump and nonbeating heart pump methods

\section{Miraki S.}

\begin{abstract}
Backgrounds: Cardiac surgery with cardiopulmonary bypass is usually done in two ways. The first method, which is more common and common, The perfusionist, with the help of the surgeon, by injection of a cardioplegia solution in the coronary arteries, causing arrested of heart rate, and the surgeon performs his own work. The second method is a Beating of the heart, and during this procedure, the heart maintains its own heart beat and the surgeon also does his job. The purpose of this study is to compare these two methods in terms of cardiac muscle damage and kidney and liver parameters and clinical outcomes in the patients.

Material and method: In this study, 43 patients that used whit cardiopulmonary bypass were divided into two Non-Beating and Beating heart groups. In the first group, 20 patients with mean age $22.35( \pm 11.31)$ and Non-Beating heart group, and in the second group 23 patients with mean age $19.78( \pm 7.57)$ The group is Beating heart. At the beginning of the referral, patients are presented with a detailed description of the method of work and informed consent. Then we measured and recorded demographics, parameters, hemodynamics and arterial blood gases, and compared them at different times.
\end{abstract}

Result: Duration of Cardiopulmonary bypass , mechanical ven- tilation and length of hospitalization in ICU were evaluated in two groups. There was no significant difference between the two groups, but the duration of operation between the two groups was different meaning (P.value $=0.003$ ) and less in Beating group. Ejection fraction (EF) was the time of discharge in the Non-Beating group $44.25( \pm 6.12)$ and in the Beating group $50.00( \pm 5.56)$, which was significantly higher in the Beating group (p-value $=0.003)$. After examining Troponin $(\mathrm{CTNI})$ and creatinine phosphokinase (CPK MB), both of 24 hours postoperatively decreased in Beating group and there was a significant difference between the two groups. Troponin with (P.value $=$ 0.023 ) and creatinine phosphokinase with (P.value $=0.038$ ). Changes in parameters of renal function (BUN, $\mathrm{Cr}$ ) and liver (ALT and AST) before, after and 24 hours after surgery in the groups under study were examined, which showed a significant difference between the two groups These variables were not observed. (p-value $>0.05$ ) Changes in parameters of arterial blood gas (ABG) such as PH, PO2, PCO2, Hgb, HCT, Na, K and Lactate before surgery, postoperatively, entering the ICU and 24 hours after surgery in the group The results of this study showed that there was no significant difference between the two groups in terms of these variables, but the $\mathrm{K}$ level after operation $4.18( \pm 0.85)$ was significantly lower (P-value $=0.037$ ) in the Beating group. Two groups were compared for inotropic drugs during surgery in the operating room and then at the intensive care unit. There was no significant difference between the two groups regarding the variables (P.value $>0.05$ ) but the rate The intake of milrinone after surgery in the intensive care unit was significantly lower (P.value $=0.024)$ in the Beating heart group.

Conclusion: From this study, it can be concluded that performing pulmonary dysfunction surgery whit cardiopulmonary bypass Non Beating and Beating heart surgery in the evaluation of liver and kidney enzymes, duration of admission to intensive care unit, duration of mechanical ventilation, there is no significant difference between them But the surgical procedure beating heart, have a better ejection fraction (EF) in discharge time and troponin and creatinine phosphokinase levels are less than 24 hours after surgery and a shorter duration time of surgery.

\section{Cardiac Surgery in Pregnancy}

\section{Ghods K. ${ }^{1}$, Aghaamoo Sh. ${ }^{2}$}

${ }^{1}$ Cardiac Surgeon, Associate Professor, Semnan University of Medical Sciences, Semnan, Iran

${ }^{2}$ Obstetricians and Gynecologists, Assistant Professor, Abnormal Uterine Bleeding Research Center, Semnan University of Medical Sciences, Semnan, Iran

\section{Abstract}

Management of pregnant women with heart disease who needs cardiac surgery intervention, is still a challenging issue for health care providers and the treatment is controversial and doubtful for cardiologist, obstetricians and gynecologists and cardiac surgeon.

Our case is an obese 35 -years- old multigravida pregnant woman with gestational age of 38 weeks (BMI=28). After the second pregnancy two years earlier, her attending physician had recommended for cardiac surgery consultation and performing of replacing the mitral valve, but the patient refused and even took no medications. She was hemodynamically stable with normal sinus rhythm and history of dyspnea on exertion (NYHA Class 2 or 3 heart disease) since second trimester. In physical examination, heart sound revealed normal S1 and S2 with mild systolic murmur on mitral position and near normal chest x-ray. 
The transthoracic echocardiography findings are: $\mathrm{EF}=50 \%$, sever eccentric mitral regurgitation jet toward superior pulmonary veins, mild left atrial enlargement and no atrial fibrillation. After undergoing the labor pain, she was transferred to cardiac operating room, to manage her with precise cardiac and hemodynamic monitoring during labor and delivery. Heart surgery team was ready, so if occurred any sign and symptoms of cardiac failure and pulmonary edema, mitral valve replacement would be performed immediately after delivery (caesarian section or normal vaginal delivery). After one and half hour induction, labor pain was arrested and caesarian section was done with optimal, closed and invasive cardiac monitoring without necessitating to mitral valve replacement. The neonate was a healthy boy with good APGAR score. The postpartum course was uneventful and the mother was discharged with medication and post-partum surveillance. We are now planning for elective mitral valve surgery.

In our center, few patients were treated without need to open heart surgery and by medical treatment and careful care of preg- nant patients with heart disease and there was not any case who requiring simultaneously to cardiac surgery and delivery. Of course, in the other hospital in our region, 2 pregnant women with extensive pulmonary thromboembolism was medically treated by cardiologist and received streptokinase, although due to unresponsiveness to streptokinase, eventually, the patients were expired resulting from cardiovascular collapse. In these cases there was no any consultation from cardiac surgeon for pulmonary thromboembolectomy.

The above mentioned case is one of many cases who we are encountered in daily practice. Many pregnant cases with known heart disease are being managing non-surgically and have a good result and outcome for mother and her neonate. However, because of multidisciplinary management of cardiac problem during pregnancy, presence of a cardiac surgeon in this setting is mandatory and should be a part of team-working in order to obtain a better outcome. The cardiac surgery efforts in pregnant women with cardiac disorders is essentially accepted in literature despite the fearfulness of unwanted side effects for mother and fetus. 


\section{Posters}

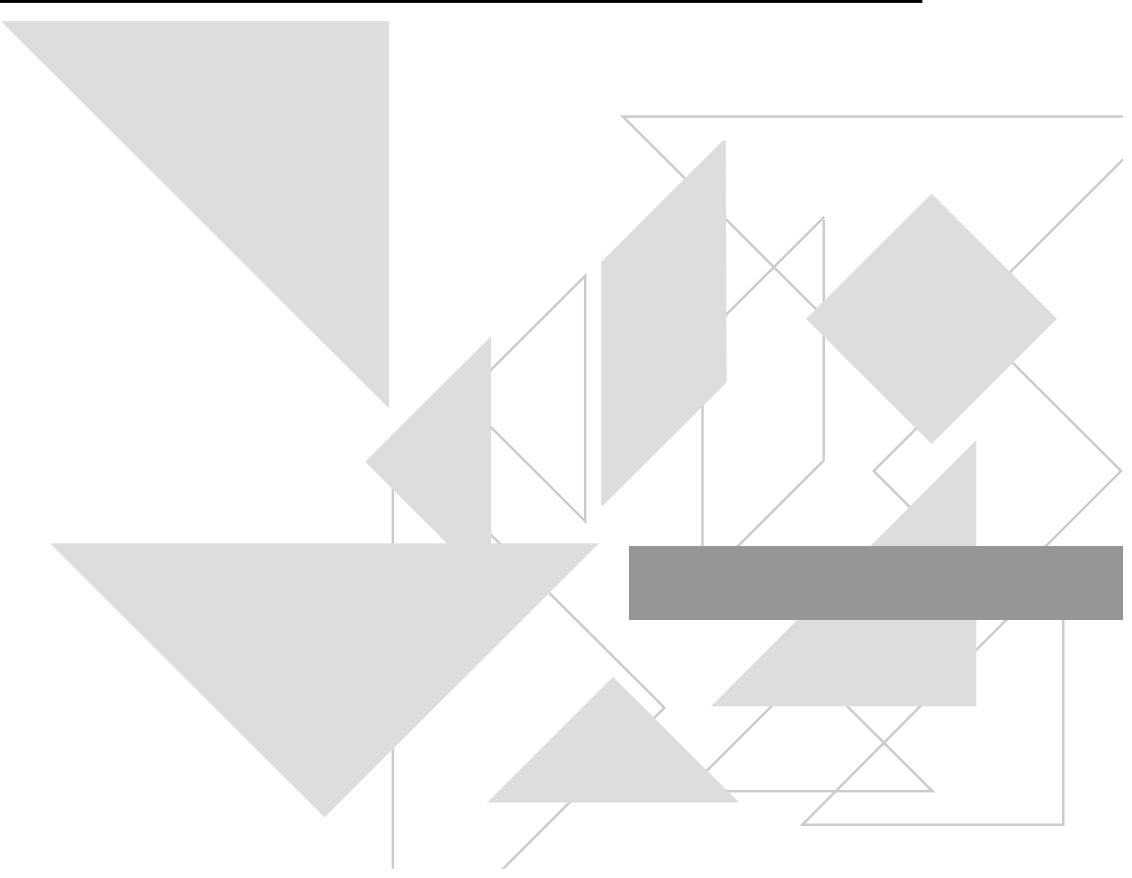




\section{- Hypertension prevalence and its association with anthropometric indices among Iranian adults}

\author{
Vakhshoori M. ${ }^{1}$, Eghbalibabadi M. ${ }^{2}$, \\ Shafieyoon S. ${ }^{3}$, Feizi A. ${ }^{4}$, Heidarpour M. ${ }^{5}$, \\ Shafie D. ${ }^{6}$, Khosravi A. ${ }^{7}$, Sarrafzadegan N. ${ }^{8}$ \\ ${ }^{1}$ Isfahan Cardiovascular Research Institute, Department Of Cardiology, Isfahan, Iran \\ ${ }^{2}$ Nursing And Midwifery Care Research Center, School of Nursing And Midwifery, Is- \\ fahan, Iran \\ ${ }^{3}$ Isfahan University Of Medical Sciences, Medical Faculty, Isfahan, Iran \\ ${ }^{4}$ Hypertension Research Center, Department Of Biostatistics And Epidemiology, Isfa- \\ han, Iran \\ ${ }^{5}$ Isfahan Endocrine And Metabolism Research Center, Department Of Endocrinology, \\ Isfahan, Iran \\ ${ }^{6}$ Heart Failure Research Center, Department Of Cardiology, Isfahan, Iran \\ ${ }^{7}$ Interventional Cardiology Research Center, Department Of Cardiology, Isfahan, Iran \\ ${ }^{8}$ Cardiovascular Research Center, Department Of Cardiology, Isfahan, Iran
}

\begin{abstract}
Backgrounds: Overweight and obesity are risk factors influencing in pathogenesis of hypertension (HTN) in which there are different methods available assessing them. The aim of our study was investigating the relation between HTN and anthropometric indices including body mass index (BMI), waist circumference (WC), waist to height ratio (WHtR), hip circumference (HC) and waist to hip ratio (WHR) among Iranian adults.

Material and method: From August 2015 till March 2016 tota numbers of 1178 males and 1086 females who aged at least 18 years were recruited in study. Demographic data were gathered through questionnaire. Blood pressure (BP) was assessed three times with one minute interval from each participant's right arm and the mean of 2nd and 3rd was considered as individual's BP. All related anthropometric indices measurements were performed with standardized equipment and by trained personnel. Result: HTN prevalence was 21.3 and 18.7 \% in men and women, respectively. All anthropometric indices had significant correlation with systolic and diastolic $\mathrm{BP}(\mathrm{P}<0.001)$. In comparison with healthy individuals, hypertensive ones had higher means of all in dices $(\mathrm{P}<0.001)$. Increased in quartiles of BMI, WC, WHtR, HC and WHR was associated with higher prevalence of HTN compared with reference group in crude model. In the last model and after adjustment of all confounding variables, these relations were remained significant especially in 3rd and 4th quartiles.

Conclusion: Our data suggested by increasing anthropometric indices, HTN prevalence would be raised. Multiple prospective studies required defining the exact relation between aforementioned variables.

\section{Amazing in hospital primary percutaneous coronary intervention results in Farshchian Heart Center}

\author{
Naghshtabrizi B. ${ }^{1}$, Emami F. ${ }^{1}$, Yazdi A. ${ }^{1}$, \\ Hosseini K. ${ }^{1}$, Mozayanimonfared A. ${ }^{1}$ \\ ${ }^{1}$ Farshchian Heart Center, Cardiology, Hamadan, Iran
}

\author{
Abstract \\ Backgrounds: This study was performed to evaluate the in hos-
}

pital outcome of PPCI for STEMI patients in referral Farshchian Heart Center, Hamadan.

Material and method: All patients with STEMI [ 1286 cases: 457 in 2016, 453 in 2017 and 376 in 2018] who were admitted to emergency room of Farshchian Heart Center between April 2016 and December 2018 underwent invasive approach, consecutively. Standard radial approach has been used in the most of the patients. In hospital mortality was assessed.

Result: The mean age of the patients was 60.5 years old (between 32 and 93 years old). The $53.5 \%$ of them were male. On admission, the mean duration of onset of symptoms to hospital arrival was $204 \pm 156.2$ minutes. The mean Door - To - Device was 78.2 minutes and the average time between ECG verification of STEMI to device time was 61.8 minutes. Stent deployment was performed in 1112 (86.46\%) cases and the rest of them either were candidated for CABGs or medical treatment. All-cause mortality occurred in $4.18 \%$ (4.36\% in 2016, $4.9 \%$ in 2017, and 3.29\% in 2018) and PPCI mortality happened in $1.71 \%$ (1.58\% in 2016, 2.3\% in 2017 and $1.25 \%$ in 2018) of patients.

Conclusion: In compare to other previous studies, the results of PPCI in our center were amazing. PPCI is feasible and efficient in STEMI patients with good out come.

\section{- Serum Vitamin D level and Hypertensive Crises}

\author{
Amanollahi F. ${ }^{1}$, Moazenzadeh M. ${ }^{2}$, \\ Rashidinejad H. ${ }^{2}$, Safizadeh H. ${ }^{3}$
}

\footnotetext{
${ }^{1}$ Clinical Research Unit, Cardiovascular Research Center, Kerman, Iran

${ }^{2}$ Basic And Clinical Physiology Sciences, Cardiovascular Research Center, Kerman, Iran ${ }^{3}$ Institude For Futures Studies In Health, Social Determinants Of Health Research Center, Kerman, Iran
}

\section{Abstract}

Backgrounds: Vitamin D deficiency is associated with hypertension; however, there is no study between vitamin D deficiency and hypertensive crises. This study was determined the relationship between serum vitamin D levels and hypertensive crises.

Material and method: This descriptive-analytical, cross-sectional study was conducted on 120 individuals within the age range of 40-80 years, selected through simple random sampling.The research population was divided into two groups of hypertensive crises $(\mathrm{n}=60)$ with blood pressure over $180 / 120 \mathrm{~mm} /$ $\mathrm{Hg}$ and hypertensive patients without history of hypertensive crises(n=60). $5 \mathrm{ml}$ peripheral venous blood samples were collected. The blood serum was isolated by centrifugal device and analyzed by ELISA.

Result: The mean levels of vitamin D in hypertensive and non-hypertensive crises groups were 29.73 and $30.23 \mathrm{ng}$ । ml,respectively. There was no significant difference between the two groups in terms of vitamin D levels ( $P>0.05)$. However no statistically significant difference was observed between the two groups considering gender $(\mathrm{P}>0.05)$. Serum vitamin D levels showed a direct correlation with the duration of hypertension and age $(\mathrm{P}<0.05$ and $\mathrm{P}<0.01$, respectively $)$.There was a significant correlation between serum vitamin D levels and the number of hypertensive crises over the past year. In this regard, the reduction of serum vitamin D levels was accompanied with the enhancement of the number of hypertensive crises $(\mathrm{P}<0.01)$.

Conclusion: There is evidence on the relationship between serum vitamin D levels and cardiovascular diseases, including hypertension. Nonetheless, in the present study, no significant 
relationship was observed between serum vitamin D level and hypertensive crises.

\section{The role of genetic counselling and familial screening in the management of Hypertrophic Cardiomyopathy}

\author{
Emrahi L. ${ }^{1}$, Shahbazi S. ${ }^{1}$, Toufan Tabrizi M. ${ }^{2}$ \\ ${ }^{1}$ Tarbiat Modares University, Department Of Medical Genetics Faculty Of Medical Sci- \\ ences, Tehran, Iran \\ ${ }^{2}$ Tabriz University Of Medical Sciences, Cardiovascular Research Center, Tabriz, Iran
}

\begin{abstract}
Backgrounds: Hypertrophic cardiomyopathy(HCM) is the most prevalent cardiomyopathy demonstrating the spectrum of genotype and phenotype heterogeneity. It is commonly inherited in an autosomal dominant pattern involving several genes. Clinical presentation varies considerably, ranging from absent or late onset to serious symptoms including angina and syncope. Owing the fact that the first manifestation may be sudden cardiac death (SCD), early identification of at risk individuals is of particular importance. This may include clinical evaluation, echocardiography, and genetic counselling and molecular screening . In this study, We aimed to bring the benefits of genetic counseling to the attention of clinicians for early diagnosis and appropriate management of HCM.

Material and method: Four HCM Patients were referred to genetic counselling after echocardiography in Shahid Madani Hospital, Tabriz, Iran. Pedigree of families was made by Progeny software and suspected family members were referred to echocardiography.

Result: In all families, there was one affected person before genetic counselling. In two families, pedigrees showed autosomal dominant inheritance with history of SCD in first degree relatives. Echocardiography of siblings revealed another affected member in these families. Also, autosomal dominant pattern was in one family that echocardiography revealed HCM condition in one of the proband;s child. Pedigree of a family with consanguineous marriage showed autosomal recessive pattern of inheritance. All children were unaffected in echocardiography assay however genetic testing was offered for all of them.

Conclusion: According to this results, genetic counselling and genetic evaluation of first degree of affected patients is impor-
\end{abstract} tant to risk stratification and manage the at risk members.

\section{The relationship between socioeconomic level and cardiovascular risk factors in patients with severe coronary angiography}

\author{
Azdaki N. ${ }^{1}$, Moezi S. ${ }^{1}$, Niroomand M. ${ }^{2}$, Khosravi \\ Bizhaem S. ${ }^{1}$ \\ ${ }^{1}$ Cardiovascular Diseases Research Center Birjand University Of Medical Sciences- \\ birjandiran, Razi Hospital, Birjand, Iran \\ ${ }^{2}$ Student Research Committee Birjand University Of Medical Sciences Birjand Iran, \\ Razi Hospital, Birjand, Iran
}

\section{$\underline{\underline{\text { Abstract }}}$}

Backgrounds: Coronary artery disease is the most important cause of death due to cardiovascular diseases and the main treatment is angioplasty. The purpose of this study was to examine the relationship between cardiovascular risk factors and socioeconomic status with the severity of coronary artery disease in patients.The extension of coronary artery disease was assessed using SYNTAX score.

Material and method: This cross-sectional study was conducted on 493 patients referring to the department of angiography in Vali-e-Asr Hospital, Birjand(Aug-Dec 2017). Demographic information, cardiovascular risk factors and socioeconomic status were assessed by using a case file and a questionnaire. Data were analyzed using Chi-square, ANOVA, Kruskal wallis tests and ziroinflated regression model by SPSS (V. 22) and R(V.3.2.1) softwares. P-value $<0.05$ considered to be significant.

Result: Mean age was $60.2 \pm 10.92$ years (30-89 years) and 50.9\% were male. The prevalence of cardiovascular risk factors was low physical activity $91.7 \%$, abdominal obesity $69.4 \%$, dyslipidemia $60.3 \%$, hypertension $46.2 \%$, diabetes $22.1 \%$, family history $22.9 \%$, opium consumption $22.5 \%$, and smoking $15.5 \%$. Mean SYNTAX score was $9.4 \pm 12.9$ and $46 \%$ of patients had score $=0,35.7 \%$ score $1-22,11.8 \%$ score $22-33$ and $6.5 \%$ score over 33 , respectively. SYNTAX score was significantly high in ageing, male and diabetic patients (OR: 1.03, 2.24, 2.69), also higher in low socioeconomic patients.

Conclusion: Given the high prevalence of cardiovascular disease and risk factors taking measures to prevent and control risk factors, and lifestyle modification including increasing the awareness and implementing preventive measures against substance abuse and improvement of quality of life and socioeconomic level seems to be necessary.

\section{Do patients with coronary artery bypass grafts using radial artery need special nursing care}

\author{
Nasrizadeh Moghaddam S. \\ ${ }^{1}$ Dena Hospital, Cardiac Surgery, Shiraz, Iran
}

\section{Abstract}

Backgrounds: The use of radial artery as bypass grafts interest to cardiac surgeons.

This is due to the improved patency rate of the radial artery grafts than that of the saphenous vein.

Material and method: Such patients may suffer complications such a edema, hematoma, pressure on the sutures causing ischemic limb problems, functional disabilities and bleeding. Result: This led us to share our experiences regarding the results of nursing care in the cardiac surgery department of Dena hospital in Shiraz, which included 600 patients undergoing coronary artery bypass graft surgery using radial artery.

Conclusion: Specific consideration in these patients such as elevation of the patients' hands ,

checking the circulation of the hand with a pulse oximeter, regulary checking of the donor site for evaluation of the hematoma, checking of the patients' performance and giving patients the necessary explanations resulted in least explanation encountered in such a large cardiac surgery cohort.

Keywords: cardiac surgery, radial artery, nursing care 


\section{Comparison of mean 2nd and 3rd blood pressure measurements with other reading frequencies among Iranian adults}

\author{
Vakhshoori M. ${ }^{1}$, Shafie D. ${ }^{2}$, Eghbalibabadi M. ${ }^{3}$, \\ Heidarpour M. ${ }^{4}$, Garak Yaraghi M. ${ }^{2}$, Khosravi A. ${ }^{1}$ \\ ${ }^{1}$ Isfahan Cardiovascular Research Institute, Department Of Cardiology, Isfahan, Iran \\ ${ }^{2}$ Heart Failure Research Center, Department Of Cardiology, Isfahan, Iran \\ ${ }^{3}$ Nursing And Midwifery Care Research Center, School of Nursing And Midwifery, Is- \\ fahan, Iran \\ ${ }^{4}$ Isfahan Endocrine And Metabolism Research Center, Department Of Endocrinology, \\ Isfahan, Iran
}

\section{Abstract}

Backgrounds: Appropriate frequencies of blood pressure (BP) measurements required to be investigated in order to find real hypertension (HTN) cases. In this article, we aimed comparing $2^{\text {nd }}$ and $3^{\text {rd }}$ time mean $B P$ measurements with $1^{\text {st }}\left(\mathrm{BP}_{1}\right), 2^{\text {nd }}\left(\mathrm{BP}_{2}\right)$, mean of $1^{\text {st }}$ and $2^{\text {nd }}\left(\mathrm{BP}_{12}\right)$ and all three ones $\left(\mathrm{BP}_{12,3}\right)$ among Iranian adults.

Material and method: A total number of 2264 individuals living in Isfahan, Iran and aged at least 18 years were recruited for study. after 5 minutes of resting in a quiet room, BP was assessed from each individual's right arm via standard equipment and methods by trained personnel. Mean $2^{\text {nd }}$ and $3^{\text {rd }} \mathrm{BP}$ was considered as reference $\left(\mathrm{BP}_{23}\right)$.

Result: Mean age of male and female participants were $41.3 \pm$ 16.5 and $40.6 \pm 15.8$ years, respectively. Both systolic blood pressure (SBP) and diastolic blood pressure (DBP) was significantly higher in men comparing with women by any measurement frequency methods. Clinically significant difference ( $\geq 5 \mathrm{mmHg}$ ) was mostly observed in systolic and diastolic of $\mathrm{BP}_{1}$ in comparison to the reference group. DBP of $1^{\text {st }}$ time measurements and all SBPs except SBP ${ }_{2}$ showed positive differences from diastolic and systolic of $\mathrm{BP}_{23}$, respectively. Furthermore, both $\mathrm{BP}_{1}$ indices revealed significant abnormal levels ( $\geq 140 / 90 \mathrm{mmHg}$ ) more frequently compared to $\mathrm{BP}_{23}$. Mean of all measurements did not show any significant relation.

Conclusion: $\mathrm{BP}_{23}$ would be a reasonable suggestion due to better categorization of HTN status and it is advisable not to consider 1st time reading for HTN diagnosis. Further studies required quantifying precise BP measurement frequencies.

\section{How to approach a case of complication "EVAR" after 5 years}

\author{
Nazari M. ${ }^{1}$, Moini M. ${ }^{1}$, Moini M. ${ }^{1}$, Moini M. ${ }^{1}$, \\ Nazari M. ${ }^{1}$, Nazari M. ${ }^{1}$, Nazari M. ${ }^{1}$ \\ ${ }^{1}$ Sina Hospital, Vascular Surgery, Tehran, Iran
}

\begin{abstract}
Backgrounds: The patient is 57 years old male with history of Diabetes,HTN, CABG ,ESRD from 1 years ago and EVAR for a 70mm infrarenal AAA with a Suitable landing zone $(2.5 \mathrm{~cm}) 5$ years ago. he was refered to Sina hospital with a complaint of generalized abdominal pain, extended to the left side of the abdomen \&
\end{abstract}

flank in the past 4 days. In the beginning of the admission BP : 160/100 PR:67 RR:18 ,Cardiopulmonary examination was normal. There was a palpable pulse and a mild tenderness in abdominal examination.Upper and lower limbs were not edematous nor cyanotic , with full and symmetric pulses. Regarding the history of aortic repair by the EVAR method.CT angiography was done from the aortic arch to the distal of both limbs. The CT angiography revealed that The graft stent detached from the landing zone and Size of aneurysm was $9.5 \mathrm{~cm}$ with severe angulation of the neck. Due to his age, low cardiac output and ESRD , The patient's desire, endovascular intervention preferred.then the patient was underwent EVAR + CHIMMNEY)Celiac artery and SMA stent graft (were performed.

\section{Angiographic evaluation of graft patency in patients with prior CABG refered to north of Iran between 2013-2017}

\author{
Ranjbar M. ${ }^{1}$, Jalali F. ${ }^{2}$, Vafai H. ${ }^{3}$, Jafaripoor I. ${ }^{2}$ \\ ${ }^{1}$ Rohani Hospital Babol University Of Medical Science, Cardiology Department, Babol, Iran \\ ${ }^{2}$ Rohani Hospital Babol University Of Medical Science, Department Of Interventional \\ Cardiology, Babol, Iran \\ ${ }^{3}$ Rohani Hospital Babol University Of Medical Science, Department Of Cardiovascular
} Surgery, Babol, Iran

\section{Abstract}

Backgrounds: Ischemic heart disease is the leading cause of death in the world. CABG (coronary artery bypass graft) is one of the standard treatment for Ischemic heart disease. Graft patency is affected by several factors. This study is conducted to find out more about these patients and the factors affecting graft patency. Material and method: This cross-sectional study is conducted to evaluate 225 symptomatic post-CABG patients, by coronary angiography from 2013-2017 in Rohani Hospital.

Result: 225 patients, including 130 men and 95 women with average age of $64 \pm 9.9$ years, underwent coronary angiography after $7.6 \pm 7$ years after being symptomatic. $71.1 \%$ of patients, had graft failure in at least one of arterial or venous grafts. The failure rate of LIMA (left internal mammary artery) to LAD (left anterior descending) did not increase with the time interval of the CABG to angiography. The rate of venous graft failure after 10 years significantly increased (p-value: 0.02 ). The incidence of LIMA to LAD patency in women $(64.7 \%)$ was less than in men $(79.9 \%)$ )p-value: 0.01 (. But the patency of venous grafts did not differ significantly between men and women (p-value: 0.8 ).

Conclusion: patency rate of LIMA to LAD graft in women was less than men and less than expected. None of the factors like diabetes, hypertension, dyslipidemia, and renal failure alone did not increase the likelihood of failure.

\section{Title A rare complication of PCI Percutaneous Coronary Intervention that leaded the patient to CABG Coronary Artery Bypass Grafting}

\author{
Salehi P. ${ }^{1}$, Alemzadeh Ansari M. ${ }^{1}$, Norouzi Z. \\ ${ }^{1}$ Rajaie Heart Center, Cardiology, Tehran, Iran
}




\begin{abstract}
Backgrounds: A 60 year old male was presented with typical chest pain NYHA (New York Heart Association) function class III. He mentioned a history of hypertension and hyperlipidemia, stroke and gastrointestinal bleeding. Angiographic results including totally cut of LAD (Left Anterior Descending) artery in midportion, totally cut of at ostial portion of the second branch of diagonal and a segmental significant stenosis in the mid portion of LCX (Left circumflex) and also a significant midportion lesion in RCA (Right Coronary Artery). He was a candidate for multivessel PCI (Percutaneous Coronary Intervention).

Material and method: Procedure including the developing of two stents in LAD: 3-33 mm Xience stent at proximal part, and a 2.5-18 Xience stent at midpart portion and balloon angioplasty on diagonal. After discharge the patient experienced chest pain again and it was continuing for six weeks. He was under medical treatment. On the next angiography for staged PCI, an unexpected pseudo-aneurysm was seen. It was in the mid part of LAD exactly on the location of the stent that was performed in the previous PCI. Due to the high possibility of thrombosis in LAD and the presence of a lesion in RCA and LCX, CABG (coronary artery bypass grafting) was considered. Result: Science LAD was patent and in this situation, using a LIMA (Left Internal Mammary artery) graft would be unsuccessful due to its occlusion by the competitive blood flow by LAD. So on the surgery, at first totally occlusion of LAD by suturing at the site of the pseudo-aneurysm, was done. Then four grafts were considered including LIMA to LAD and three SVG (Saphenous Vein Graft) grafts to OM1 (Obtuse Marginal), D1 (Diagonal) and PDA (Posterior descending artery).

Conclusion: Coronary aneurysm is a rare complication after PCI and it can be life-threatening. Early diagnosis can be lifesaving. Chest pain after any interventions is a leading point to diagnose the complications, especially when there is no response to the medical treatment. Regular follow up is the key point to diagnose any complications in the golden time for the treatment.
\end{abstract}

\section{Novel distal accesses in the hand for percutaneous coronary angiography and intervention}

Roghanidehkordi F. ${ }^{1}$, Hashemifard O. ${ }^{1}$, Sadeghi M. ${ }^{1}$, Mansouri R. ${ }^{1}$, Akbarzadeh M. ${ }^{1}$, Dehghani A. ${ }^{2}$, Akbari M. ${ }^{3}$

${ }^{1}$ Isfahan University Of Medical Sciences, Cardiology, Isfahan, Iran

${ }^{2}$ Islamic Azad University, Nursing And Midwifery, Isfahan, Iran

${ }^{3}$ Isfahan University Of Medical Sciences, Biostatistics, Isfahan, Iran

\section{Abstract}

Backgrounds: Trans-radial and trans-ulnar accesses have been practiced and recommended as default and alternative techniques for coronary angiography and angioplasty in recent years. In this study, we present new innovative approaches using more distal access points, i.e. trans-snuff box and trans-palmar approaches.

Material and method: We conducted dorsal hand access (transsnuff box) for angiography and/or angioplasty on 235 patients since 1 October 2016 and trans-palmar access (superficial palmar branch of ulnar artery) on 175 patients since 15 October 2016 in Shaheed Chamran, Khorshid and Shaheed Saddoghi hospitals (Isfahan, Iran).

Result: In 221 patients out of 235 ones (94.1\%)(men=76.5\%, mean \pm SD of age $=57.4 \pm 10.4$ years and women $=23.5 \%$, mean \pm SD of age $=62.4 \pm 9.5$ years), our procedure through snuff box (dorsal hand )was successfully performed .In 159 patients out of 175 ones (90.8\%) men $=76 \%$, mean \pm SD of age $=58.1 \pm 10.5$ and women $=24 \%$, mean \pm SD of age $=61.2 \pm 9.6$ ) our procedure through palmar artery was successfully performed. In total, the evaluated patients had mild pain 3.4\% for snuff box and $4.5 \%$ for palmar), ecchymosis in distal forearm (5.1\% for snuff box $2.8 \%$ for palmar), with no major complications even one (amputation, infection, thrombosis, need for surgery, hand dysfunction, nerve palsy, and so forth).

Conclusion: Although our procedures are at their early stages with about a follow-up period of 17 months, more researches are recommended to be conducted in forthcoming months \&years and this new innovative approaches could be suggested safe, feasible \& reliable with low complications.

\section{Study of Correlation between Vitamin D and Coronary Artery Calcification}

Sajjadieh Khajouei A. ${ }^{1}$, Sajjadieh Khajouei H. ${ }^{1}$, Kasaii Z. ${ }^{2}$

${ }^{1}$ Isfahan University Of Medical Sciences, Internal Medicine Department, Isfahan, Iran

2 Isfahan University Of Medical Sciences, Pathology Department, Isfahan, Iran

\section{Abstract}

Backgrounds: considering the role of vitamin D in cardiovascular disease (CVD) and the relationship between coronary artery calcium score (CAC) and CVD, we aimed to investigate the association between serum level of vitamin D level and CAC.

Material and method: in this cross sectional study, 83 consecutive patients were referred for performing CT angiography to measure CAC. An educated nurse filled a questionnaire containing the information about past medical history of diabetes mellitus, hypertension, hyperlipidemia and smoking for each patient. Serum samples were collected for the evaluation of vitamin D level. After adjustment with confounders, we extracted and analyzed the data to assess relationship between vitamin D level and CAC.

Result: this study included the final number of 67 patients of which 37 had positive CAC (mean CAC: 128.1) and patients had 30 negative CAC (mean CAC: 0). There was no significant association between CAC and vitamin D levels ((Pearson coefficient $=-0.01$ and Spearman coefficient $=-0.03$, P value $>0.05$ ) but the past history of hyperlipidemia and D.M were more prevalent in CAC-patients than those without $\mathrm{CAC}$ (62.2\% and $32.4 \%$ vs. $60 \%$ and $13.3 \%$ ) (P value $<0.05$ ).

Conclusion: our study revealed that vitamin D had no significant relationship with CAC. Furthermore, among major risk factors of cardiovascular diseases, diabetes mellitus and hyperlipidemia are associated with higher CAC scores

Impact of Procaine Hydrochloride Versus Lidocaine in Cardioplegic Solution on Reperfusion Arrhythmia During Coronary Artery Bypass Graft Surgery

Sadeghi S. ${ }^{1}$, Austine N. ${ }^{2}$, Totonchi Z. ${ }^{3}$, Bakhshandeh $\mathrm{H}^{4}{ }^{4}$, Hadavand N. ${ }^{5}$, Alizadeh Ghavidel A. ${ }^{6}$ 
${ }^{1}$ Rajaei Cardiovascular Medical And Research Center, Perfusion Unit, Tehran, Iran

${ }^{2}$ Iran University Of Medical Sciences, Medical Faculty, Tehran, Iran

${ }^{3}$ Rajaei Cardiovascular Medical And Research Center, Cardiac Anesthesia, Tehran, Iran

${ }^{4}$ Rajaei Cardiovascular Medical And Research Center, Epidemiology, Tehran, Iran

${ }^{5}$ Rajaei Cardiovascular Medical And Research Center, Clinical Pharmacy, Tehran, Iran

${ }^{6}$ Rajaei Cardiovascular Medical And Research Center, Cardiac Surgery, Tehran, Iran

\section{Abstract}

Backgrounds: The best way to reduce the occurrence of arrhythmias that generally occur after an open heart surgery is to improve the quality of myocardial protection against reperfusion damage during the cross-clamp time. In this regard, different cardioplegic solutions play a key role. Differences in the types and contents of cardioplegic solutions may lead to different results of effective protection of the myocardium. The purpose of this study was to compare the effects of the newly introduced procaine hydrochloride (PHC) containing cardioplegic solution (Shahid Ghazi Pharmaceutical Co. Tabriz, Iran) and lidocaine (L) in cardioplegic solution on post aortic clamp arrhythmia in coronary artery bypass graft surgery.

Material and method: This study is a randomized clinical trial that was performed in Rajaie cardiovascular, Medical and research center after obtaining permission from the ethics committee. A total of 100 patient candidates for coronary intervention from October 2016 to March 2017 were divided into two groups and randomly assigned one of the two cardioplegic solutions that were only different in the antiarrhythmic solution contents of procaine hydrochloride and lidocaine. After aortic de-clamp during post ischemic time, spontaneous sinus rhythm return, post-operative arrhythmia, and the dosage of lidocaine, magnesium and inotropic drugs, or the use of defibrillator and pacemaker in the operating room or the intensive care unit have been investigated.

Result: After aortic de-clamp, in the PHC group, the spontaneous return of heart rate was higher ( $\mathrm{P}$ value $=0.02,64 \%$ for $\mathrm{PHC}$ and $42 \%$ for L group). Furthermore, the required dosage for lidocaine and magnesium $(\mathrm{P}=0.02)$ and inotrope $(\mathrm{P}=0.04)$ were also relatively lower, but in general, the percentage of arrhythmia occurred requiring defibrillator and pacemaker after operation between the two groups did not show any significant differences. Clinically, the required cardioplegic solution volumes were slightly higher in PHC group.

Conclusion: Although the cardioplegic solution containing procaine hydrochloride is effective in protecting myocardium during non-complex surgeries to spontaneously reverse the heart rhythm, it does not have any significant effects on decreasing arrhythmia after aortic de-clamp and is not preferable to the cardioplegic solution containing lidocaine. Obviously, the definitive result depends on the repetition of the results based on similar clinical studies, with more patients.

\section{Resternotomy a single center experience}

\author{
Salehi M. ${ }^{1}$, Bakhshandeh A. ${ }^{1}$, Rahmanian M. ${ }^{1}$, \\ Alemohammad M. ${ }^{2}$, Saberi K. ${ }^{2}$ \\ ${ }^{1}$ Tums Imam Khomeini Hospital, Cardiac Surgery, Tehran, Iran \\ ${ }^{2}$ Tums Imam Khomeini Hospital, Anesthesiology, Tehran, Iran
}

\section{Abstract}

Backgrounds: Every cardiothoracic surgeon is dealing with ever increasing patients who need for reoperation. These group of patients are increasing and will continue to increase as the general population ages. Reoperations are technically more difficult because of associated risk of reentry in a heart with more advanced pathology, little reserve and the presence of more frequent comorbidities. Routine peripheral cannulation in every patient who needs resternotomy is inadvisable, time consuming and has no noticeable role in decreasing the risk of reentry. In our center, before proceeding with a resternotomy, we deal with this issue by taking a lateral CXR the day before operation and according to our findings, associated patient risk factors for reoperation, previous patient operation and new pathology that we are dealing to it, intending the best approach individually. In this article we pay attention to our experiences in more than two hundred resternotomy without any peripheral cannulation.

Material and method: This is a retrospective cohort study on resternotomy patients, between June 2011 and August 2018 in Imam Khomeini Hospital Complex, Tehran University of Medical Sciences. We enrolled 437 consecutive resternotomy patients,229 (52.4\%) male and 208 (47.5\%) female, mean age of $47.7 \pm 18.2$ years. CXR was taken from every patient the day before operation and according to our findings, patient risk factors for resternotomy and previous operation, intending the best approach individually. Our goals was to observe the events intraoperatively and their outcomes after operation. Nearly we never used peripheral cannullation in our patients and resternotomy and central cannullation was the rule.

Result: Mean ICU stay was $3.1 \pm 0.9$ days and 21 (4.8\%) patients expired during hospital stay. The most common cause of death in our series was renal failure in 15 ( 71.4\%) patients, coagulopathy in $4(19 \%)$ patients and cardiac failure in $2(9.5 \%)$ patients respectively. We had three RV, one RA, one pulmonary artery and two IVC tearing during resternotomy and dissection which was controlled easily without peripheral cannullation. Femoral cannullation before resternotomy was performed in one patient who need emergency pulmonary embolectomy.

Conclusion: A relatively standard practice for resternotomy is use of peripheral cannulation technique to institute cardiopulmonary bypass prior to opening the chest. Peripheral cannulation techniques, especially in obese patients can be associated with notable comorbidities and longer hospital stay. In our experiences resternotomy and central cannullation is a safe strategy and peripheral cannullation before resternotomy should be kept for highly selective patients.

\section{- Exposure of electromagnetic field emitted by mobile phone on cardiac rhythm disorders of patients with Aortic valve replacement}

\author{
Jargouei Esparti M. ${ }^{1}$, Hutter H. ${ }^{1}$, \\ Mirmohammadsadeghi A. ${ }^{2}$, Kundi M. ${ }^{1}$ \\ ${ }^{1}$ Medical University Of Vienna, Centre For Public Health, Vienna, Austria \\ ${ }^{2}$ Isfahan University Of Medical Sciences, Cardiovascular Surgery, Isfahan, Iran
}

\section{Abstract}

Backgrounds: Several studies examined the effects of radiofrequency electromagnetic field (RF-EMF) emitted from mobile phone (MP) use on health and wellbeing, but research on the effect of MP exposure on cardiovascular system has yield con- 
troversial results. Hence the present study investigated participants who are especially susceptible to cardiac electrical impairment, namely postoperative patients with mechanical aortic valve replacement.

Material and method: This controlled experimental study was carried out on 40 male participants (17-60 years) who had undergone the procedure of mechanical aortic valve replacement. Participants were randomly divided into a control and experimental group, with the experimental group being exposed to RF from a phone call of 20 minutes duration. Three lead Holter monitors recorded heart rate, corrected QT interval and time domain heart rate variability parameters during, before and after the phone call. Blood pressure and ECG were assessed before and after the exposure period in both groups.

Result: In the experimental group, mean heart rate significantly decreased during exposure (p-value $<.05)$. The mean corrected QT interval prolonged only insignificantly during MP exposition. Several heart rate variability parameters increased significantly during exposure (p-value $<.05$ for SDNN, SDNNI, TRI and RMS-SD) in the experimental group. No dysrhythmia and significant changes in systolic and diastolic blood pressure occurred ( $\mathrm{p}$-value > 0.05)

Conclusion: The study demonstrates that RF exposure from mobile phones may change the balance of the autonomic nervous system through increasing the parasympathetic tone and decreasing the sympathetic tone.

\section{The effect of topical use heparinized own blood on post operation blood loss in patient with open heart surgery a randomized controlled trial}

\author{
Sobhanian K. ${ }^{1}$, Salehi M. ${ }^{1}$, Bakhshandeh A. ${ }^{1}$, \\ Rahmanian M. ${ }^{1}$, Al E Mohamad M. ${ }^{1}$, \\ Karamnezhad M. ${ }^{1}$ \\ ${ }^{1}$ Tehran University Of Medical Science, Cardiac Surgery, Tehran, Iran
}

\begin{abstract}
Backgrounds: Bleeding remains a common complication in the postoperative period of open heart surgery $\bullet$ The aim of this study was to investigate the effect of topical use 20cc heparinized own blood which is taken from the patient before starting $\mathrm{CPB}$ on post operation blood loss in the patient with open heart surgery.

Material and method: This study was done on 546eligible patient with open heart surgery. The samples were randomly allocated into intervention and control groups, who received heparinized ( $\mathrm{n}=273$ ) and typical blood $(\mathrm{n}=273)$, respectively $\bullet$ In intervention group ,After the reversal of the systemic heparinization and before closing the sternum, 20cc heparinized own blood which is taken from the patient before starting CPB was poured directly into the field and aspirated by the mediastinal drain tube after the complete closure of the incision

Methods $\bullet$ a parallel design, triple-blind, randomized and controlled clinical trial (RCT) was done on 546 eligible patient with open heart surgery. The samples were randomly allocated into intervention and control groups, who received heparinized $(\mathrm{n}=273)$ and typical blood $(\mathrm{n}=273)$, respectively. The blood loss was also measured in three time intervals after the open-heart surgery ( 6 hours, 24 hours and at the time of bleeding cessa-
\end{abstract}

tion)in the intervention and control groups. Data were analyzed through SPSS using descriptive statistic and repeated measurement analysis.

Result: The mean postoperative blood loss at the time of total blood loss in the intervention and control groups were $665.44 \pm 493.01 \mathrm{cc}$ and $913.39 \pm 820.92 \mathrm{cc}(\mathrm{p}=0.05)$, respectively. In a two-group analysis in 6 and 24 hours following the cardiac surgery and also, in the time of total loss blood, the control group showed a greater post-operative blood loss rate than the intervention group (heparinized blood group $(p=0.04)$.

Conclusion: in our institute we found that use 20cc heparinized own blood which is taken from the patient before starting $\mathrm{CPB}$ that was poured directly into the field and aspirated by the mediastinal drain tube after the complete closure of the incision decrease significantly the volume of postoperative blood loss, need of blood products and rate of re-exploration

\section{Some factors associated with hospital mortality in adult patients undergoing open-heart surgery}

\section{Sobhanian K. ${ }^{1}$, Salehi M. ${ }^{1}$, Bakhshandeh A. ${ }^{1}$, Rahmanian M. ${ }^{1}$, Bakhtiari A. ${ }^{2}$, Al E Mohamad M. ${ }^{1}$, Kassani A. ${ }^{3}$}

${ }^{1}$ Tehran University Of Medical Sciencetums, Cardiac Surgery, Tehran, Iran
${ }^{2}$ Dezful University Of Medical Science, Emergency Medicine, Dezful, Iran
${ }^{3}$ Dezful University Of Medical Science, Community Medicine, Dezful, Iran

\section{Abstract}

Backgrounds: Open-heart surgery is an invasive method that can cause severe complications and mortality in patients with heart problems. This study aimed to explore the hospital mortality rate and associated factors in adult patients undergoing open heart surgery

Material and method: A total of 668 patients from october 2016 to october 2017 were included in the study. The Mean age of the patients was $53.73 \pm 17.08$ years, and $65.24 \%$ of them were male.This cross-sectional study included all patients who admitted for openheart surgery ( $n=702)$ from october 2016 to october 2017. The main outcome (mortality) was 30 day post-operative mortality. The data collected were demographic status, smoking status, surgery type, renal failure (Creatinine $>1.5 \mathrm{mg} / \mathrm{dl}$ ), volume of blood loss, use of Plavix and hypertension.

Result: The overall hospital mortality rate was $6.43 \%(n=43)$. Mortality rate in male and female were $7.52 \%$ and $4.38 \%$ (odds ratio $[\mathrm{OR}]=1.77, \mathrm{p}=0.18$ ), respectively. Logistic regression model showed that independent risk factors for hospital mortality rate were age $(\mathrm{OR}=1.08)$, surgery type $(\mathrm{OR}=1.70)$, renal failure $(\mathrm{OR}=1.16)$, ICU length of stay ( $\mathrm{OR}=1.33$ ) and post operative blood loss ( $\mathrm{OR}=1.39$ ). Sex, smoking status, use of Plavix and hypertension had no association with mortality rate in those admitted to the hospital for open-heart surgeries.

Conclusion: Type of surgery was noted to be one of the main risk factors for hospital mortality rate as the combined surgery method had higher mortality rate than coronary artery bypass graft surgery and other surgery methods. - Also, decreasing blood loss in the post operation can be prevented from mortality in the patients undergoing open heart surgery. Keywords: Open-heart surgery, hospital mortality, risk factors. 


\section{A new method of sternal closuresternal closure with nylon tape}

\author{
Sobhanian K. ${ }^{1}$, Salehi M. ${ }^{1}$, Al E Mohamad M. ${ }^{1}$, \\ Kassani A. ${ }^{2}$ \\ ${ }^{1}$ Tehran University Of Medical Sciencetums, Cardiac Surgery, Tehran, Iran \\ ${ }^{2}$ Dezful University Of Medical Science, Community Medicine, Dezful, Iran
}

Abstract

Backgrounds: Sternal closure with wire have many complications for patients such as pain, cutting of bone ,postoperative bleeding from wire site in sternum and dehiscence of wound due to wire fracture. $\bullet$ many of these complications can increase reexploration, length of icu and hospital stay and mortality. this study hopes to comparison of efficacy and complications of two methods of sternal closure : nylon tape versus steel wire

Material and method:

- $(244+244)=488$ patients

- 244 patients in nylon tape group and 244 patients in steel wire group

- Mean age : 58 years

- Mean BMI : 25

- Diabetics : $37 \%$

- COPD : $8 \%$

- male/female :286/202

Methods : In this prospective randomized study all patients after surgery followed for 2 month about:

- Wound infection and mediastinitis

- Dehiscence of sternum

- Volume of bleeding in first post operative day

- Death

- Icu and hospital stay

- Need to reexploration for control of bleeding or tamponade In this study, unlike the wire method, we pass nylon tapes with a clamp around the sternum from intercostal spaces . thus, bone will be intact

Result:

- Volume of bleeding in first post operative day was lower in nylon type group $(\mathrm{p}=0.02)$

- Sternal healing was not significantly different in two groups

- Sternal dehiscence was lower in nylon tape group ( $\mathrm{p}=0.04$ )

- Mediastinitis and wound infection and mortality was similar in two groups

- Icu stay and hospital stay were lower in nylon $\operatorname{group}(\mathrm{p}=0.09)$

but were not statistically significant between two groups

- Reexploration for control of bleeding and tamponade was low-

er in nylon tape group $(\mathrm{p}=0.02)$

- Cost of sternal closure with nylon tape was lower than wire

- The time consuming for sternal closure was similar between two group

Conclusion: This study showed not only this new technique in sternal closure was not worse than steel wire closure but also this new innovation addedmany benefits versus wire closure for example:

- Lower hemorrage in first post operative day and lower need for reexploration for control of bleeding or tamponade

- Lower cost of nylon tape versus steel wire

- Lower rate of Sternal dehiscence

- Similar incidence of major complications such as (mediastinitis , death)

- Similar postoperative outcomes such as (icu stay and hospital stay)

\section{Prevention of Post-Operative Arrhythmias in Coronary Artery Bypass Graft OperationsUse of Magnesium Sulfate}

\author{
Rozbahani M. ${ }^{1}$, Bahremand M. ${ }^{1}$, Janjani P. ${ }^{2}$ \\ ${ }^{1}$ Medicine School Kermanshah University Of Medical Sciences, Department Of Cardiol- \\ ogy, Kermanshah, Iran \\ ${ }^{2}$ Faculty Of Social Sciences Razi University, Department Of Psychology, Kermanshah, \\ Iran
}

\section{Abstract}

Backgrounds: Arrhythmia is known as a common complication of most major surgeries. The reduction in the Serum level of $\mathrm{Mg}+2$ during the peri-operation period plays an important role in prevention of arrhythmias through stabilization of cell membrane. The aim of this study was to investigate the use of prophylactic magnesium sulphate in treating arrhythmias that may occur following coronary bypass grafting operations Material and method: In this randomized double blinded clinical trial study, the population consisted of 174 consecutive patients undergoing the Coronary Artery Bypass Grafting (CABG). 87 patients given $3 \mathrm{~g}$ of magnesium sulphate (MgSO4) [20 ml $=24.32 \mathrm{mEq} / \mathrm{L} \mathrm{Mg}+2]$ in $100 \mathrm{cc}$ of isotonic $0.9 \%$ solution over 2 hours intravenously at the following times: 12 hours prior to the operation, immediately following the operation, and on postoperative days 1, 2, and 3 (Group 1). Another group including 87 patients given $100 \mathrm{cc}$. isotonic $0.9 \%$ as placebo, during the same time periods (control group).

Result: In the postoperative period, the magnesium values reduced in control Group and increased in case group due to its injection. Arrhythmia prevalence was reduced significantly in favor of magnesium group $(\mathrm{P}=0.013)$. The two groups showed no significant differences in other operative or postoperative measurements. No side effects of the drugs were observed.

Conclusion: Prophylactic use of magnesium sulphate is effective at preventing arrhythmia that may occur following coronary by-pass operations. Magnesium sulphate should be used in prophylactic treatment since it may decrease arrhythmia at low doses. If arrhythmia should occur despite this treatment, intervention with amiodarone may be preferable.

\section{Optimizing venous drainage using a modified roller pump without oxygenator in surgical management of right heart diseases}

\section{Rahmanian M. ${ }^{1}$, Salehi M. ${ }^{1}$, Bakhshandeh A. ${ }^{1}$, Saberi K. ${ }^{1}$}

${ }^{1}$ Tehran University Of Medical Sciences, Cardiothoracic Surgey Imam Khomeini Hospital, Tehran, Iran

\section{$\underline{\underline{\text { Abstract }}}$}

Backgrounds: Right heart bypass is a form of perfusion that af ter getting the venous return from IVC, SVC and coronary sinus, send it to the pulmonary artery for oxygenation by the patient' lungs as the natural oxygenator. So with this technique, not 
only the right side of the heart is isolated and ready for any surgical intervention, but also need for oxygenator and systemic bypass is gone. Further with this technique there is no systemic response to cardiopulmonary bypass because the left heart is beating and receives its oxygenated blood from the own lung.

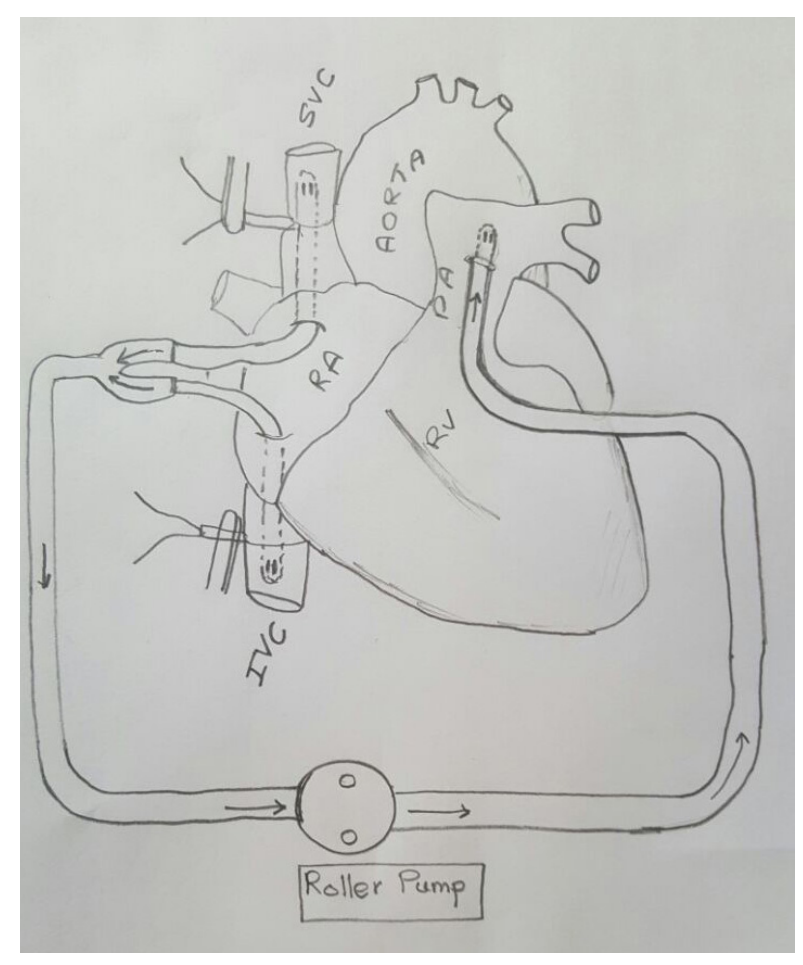

Material and method: A 29-year-old man with a history of multiple trauma was undergoing thoracotomy for repair injured lung and laparotomy for splenectomy.

Thereafter, patient needed to ICD leads due to persistent junctional rhythm. After six months, the patient developed fever that work up showed lead related endocarditis without response to antibiotic therapy and scheduled for surgical intervention.Surgical plan wasextraction of ICD lead in the right atrium with using of right heart bypass with autologous lung perfusion using a roller pump without oxygenator

Result: The patient' postoperative course was good without any complication and transferred to the other ward for completing antibiotic course.

Conclusion: We conclude that this technique is an easy, affordable, safe, reproducible technique for removal of Infected leads and all vegetation and may be extended for other surgical intervention for right side of heart (such as resection of RA rumor or clot). Also, our technique is less invasive physiologically and useful in patients with preoperative sepsis and marginal cardiopulmonary function.

\section{What do you know about your child's disease A Rough Assessment of Parents' knowledge about Their Child's Congenital Heart Disease}

\author{
Moradian M. ${ }^{1}$
}

\section{$\underline{\underline{\text { Abstract }}}$}

Backgrounds: In our study, Parents' information about their children's heart disease is very incomplete and is generally related to the level of parents' education. Currently, it seems like explanations provided by physicians about children's illnesses does not have an effective role in raising awareness for parents, and parents with lower levels of education cannot benefit from doctors' explanations

Material and method: During three months, we enrolled 104 patients who were visited in pediatric cardiology outpatient clinic of Rajaie Cardiovascular, medical, and research center in Tehran. Questionnaires containing five basic as well as important questions were presented to their parents.

Result: Statistical tests showed that, there is a significant relationship between mother's education, father's education, history of performing angiography or surgery with parental information about the name of heart disease, the ability to show the location of a child's defect on a schematic image of the heart, knowledge of heart disease symptoms and awareness about the causes of congenital heart disease $(p<0.05)$.

Conclusion: In our study, Parents' information about their children's heart disease is very incomplete and is generally related to the level of parents' education. Currently, it seems like explanations provided by physicians about children's illnesses does not have an effective role in raising awareness for parents, and parents with lower levels of education cannot benefit from doctors' explanations

\section{A rare coronary artery variant super- dominant Left Anterior Descending coronary artery}

\author{
Pouraliakbar H. ${ }^{1}$, Rezaeian N. ${ }^{2}$, Najafi H. ${ }^{2}$, Bayati P. ${ }^{3}$, \\ Khaki M.
}

${ }^{1}$ Shaheed Rajaei Cardiovascular Medical And Research Center, Department Of Radiology, Tehran, Iran

${ }^{2}$ Shaheed Rajaei Cardiovascular Medical And Research Center, Department Of Cardiovascular Medicine, Tehran, Iran

${ }^{3}$ Shaheed Rajaei Cardiovascular Medical And Research Center, Department Of Cardiac Imaging, Tehran, Iran

\section{Abstract}

Backgrounds: The posterior descending artery originates either from the right coronary artery or the left circumflex artery. We present a rare case of hyper-dominant left anterior descending(LAD)coronary artery continuing as the posterior descending artery beyond the crux.

Material and method: A 51-year-old man with a history of hypertension and smoking, presented with a new onset of atypical chest pain. Physical examination was unremarkable. Diagnostic tests, including blood tests, electrocardiography, echocardiography, exercise stress test and also Coronary computed tomography angiography were done.

Result: Electrocardiography was normal, exercise stress test was borderline and echocardiography showed evidence of normal biventricular function. Coronary computed tomography angiography showed a normal left main coronary artery. Our patient had a rare coronary artery anomaly with an unusual left anterior descending course through the interventricular septum before it approached the posterior atrioventricular groove.The LAD 
coronary artery was normal, without luminal stenosis. The left circumflex (LCX) artery was giving rise to two obtuse marginal branches and terminated to the small posterior left ventricular branch. The right coronary artery was rising normally from the right aortic sinus of Valsalva and was a non-dominant artery. Conclusion: Stenosis of super-dominant LAD may have catastrophic complications, including early and delayed ruptures of the right ventricular free wall and ventricular septum and rupture of the left ventricle aneurysm. Our patient showed no evidence of coronary artery involvement, but had hypertension as a risk factor of coronary atherosclerosis. Regarding the complicated course of artery stenosis side effects in patients with hyper-dominant LAD, aggressive management of risk factors and lifestyle improvements could prevent catastrophic results.

\section{Speckle Tracking Echocardiography Findings in Healthy Individuals with Fragmented QRS}

\author{
Nikoo M.', Abtahi F. ${ }^{1}$ Jamali Z. \\ ${ }^{1}$ Shiraz University Of Medical Sciences, Cardiology, Shiraz, Iran
}

Abstract

Backgrounds: Previous investigations reveal that Fragmented QRS (FQRS) waves in ECG have a significant role in diagnosis and prognosis of the patients with cardiovascular diseases. Most of the previous studies performed on those patients with known underling cardiovascular diseases such as previous myocardial infarction, heart failure, and myocardial scars. To our best knowledge there no previous studies in which targeted healthy people for this issue. The goal of present study was to investigate the Speckle Tracking Echocardiography Findings in Healthy Individuals with Fragmented QRS

Material and method: The ECG of the patients who obtained from the participants of the Shiraz Cohort Study was reviewed and those ECGs with FQRS were identified and the patients were asked to undergo Speckle Tracking Echocardiography. Also similar number of healthy individuals with normal ECG was recruited as control group. Data analysis was performed by SPSS software version 18 for windows. P value of less than 0.05 was considered as statisticallty significant

Result: Twenty individuals with abnormal ECG were recruited and 20 with normal ECG. The result of the investigation reveals that GLS-A4C and GLS-AVR values were significantly lower in those participants with FQRS in ECG compare to those with normal ECG. Also there was significate difference in Septal, Anteroseptal, and Inferior segments of individuals with FQRS in ECG compare to those without

Conclusion: Present study shows that accidental finding of FQRS waves in ECG of healthy people is clinically important and it is suggested that physicians who detect this in the ECG consider further cardiovascular evaluation for the patient

\section{- Frequency of Reoperation due to Bleeding after Adult Cardiac Surgery at Rajaie Heart Center}

\author{
Sadeghi A. ${ }^{1}$, Farasatkish R. ${ }^{1}$, Azarfarin R. ${ }^{1}$, \\ Ziyaeifard M.', Faritous Z. ${ }^{1}$, Hadipoorzadeh F. ${ }^{1}$ \\ ${ }^{1}$ Rajaie Heart Center, Anesthesiology, Tehran, Iran
}

\section{Abstract}

Backgrounds: Reoperation due to bleeding in adult cardiac surgery is an important complication after surgery which increases mortality.Studying the risk factors and outcomes of reoperation in these patients is imperative.

Material and method: In this study , 703 adult patients undergoing cardiac surgery with cardiopulmonary bypass were examined for three months and patients who entered the study. we evaluated the frequency of re-exploration due to high chest tubes drainage or tamponade after cardiac surgery.

Result: In this study, from 703 patients studied within 3 months period, 53 patients returned to the operating room due to bleeding or tamponade. According to this study, $7.5 \%$ of adult patients returned to the operating room due to bleeding.

Conclusion: Massive bleeding following cardiac surgery may leads to re-operation and blood transfusion. This lead to increased complications and increased mortality and postoperative morbidity.

\section{-Short-term Outcome of Patients with Infective Endocarditis A Single-center Prospective Study}

\author{
Saberi K. ${ }^{1}$, Salehi M. ${ }^{2}$, Bakhshandeh A. ${ }^{2}$, \\ Rahmanian M.', Alemohammad M. \\ ${ }^{1}$ Tums Imam Khomeimi Complex, Anesthesiology, Tehran, Iran \\ ${ }^{2}$ Tums Imam Khomeimi Complex, Cardiac Surgery, Tehran, Iran
}

\section{Abstract}

Backgrounds: Although there were many developments and modifications in health care policies for more hygienic conditions, medical therapies, antibiotics, and sterility, the infective endocarditis (IE), even so, endured as one of the most problematic conditions that have a same occurrence as the past years. Despite the introduction of new antibiotics and prescription of the innovative regimens, up to $40 \%$ of the patients with such disease in contrast are candidates for surgical intervention.

Material and method: We collected data of 42 patients in Imam Hospital, which is a university-based 1700-bed center from July 2014 to June 2016. A total of 42 patients (33 males, nine females), with an average age of 42.42 (minimum 22, maximum 77) years, were candidates for different type of surgery associated with IE.Our work was confirmed by the committee on humanresearch and medical ethics of Tehran University of Medical Sciences. In addition to echocardiograms, the diagnosis of multiple valve endocarditis was approved by Duke criteria.[6] An active endocarditis was considered due to urine analysis and/or blood culture and acute inflammation Gram stains of sample tissue.

Result: Descriptive data are demonstrated as mean (minimummaximum), and continuous variables are indicated in frequency and percentage. In our patient population, there were 33 (78.6\%) males and $9(21.4 \%)$ females. The minimum age was 22 , and the maximum was 77 with an average of 42.42 years old. The mean weight was $68.46 \mathrm{~kg}$. Average ejection fraction of our patients was $40.60 \%$.Twelve patients experienced a redo operation; in which, 2 of them have had the second redo. Five patients underwent an aortic valve replacement operation, 2 MVR, and just one patient had experienced a subaortic web resection. Only 4 
patients had hypertension, and the rest had normal pressurestatus. Only 3 patients had diabetes mellitus. Seven cases were addicted; in which, 5 of them were intravenous (IV) abusers. There was one porphyric patient who suffered from pethidine reliability. Most of our cases underwent Bentall or TVR operation, and multivalve operation was more scarce;Only one patient was explored after the main operation due to bleeding, and one patient had Intensive Care Unit (ICU) readmission. From the seven patients who died, one of them expired in the odd ratio, and the rest of them expired due to probable sepsis; the detail of expired patients'

Conclusion: We have presented the therapeutic strategies and outcome of patients with IE and evaluated their short-term outcome.

\section{Effect of Remote Ischemic Preconditioning on ischemic biomarkers in Coronary Artery Bypass Graft}

\author{
Javaherforoosh Zadeh F. \\ ${ }^{1}$ Ahvaz Jundishapur University Of Medical Sciences, Cardiac Anesthesia, Ahvaz, Iran
}

\section{$\underline{\underline{\text { Abstract }}}$}

Backgrounds: Objective: Elective open heart surgery is associated with troponin release in some cases due to myocyte necrosis. The aim this study was to measure perioperative cardiac troponin I (cTnI) in elective coronary artery bypass graft (CABG) after remote ischemic preconditioning

Material and method: Methods: Twenty-eight patients were selected for elective CABG. They were randomized to receive remote ischemic preconditioning (induced by three 5-min cycles of inflation with a pneumatic tourniquet and 5-min deflation between inflation episodes as reperfusion). Outcomes: Primary outcomes were cardiac troponin I levels at 6 and $24 \mathrm{~h}$ after procedure, and the secondary outcomes included creatine phosphokinase, lactate dehydrogenase and serum creatinine levels. Hemodynamic changes were evaluated between the treatment and control groups.

Result: Results: Cardiac troponin I at $6 \mathrm{~h}$ after preconditioning was significantly lower than in the control group $(\mathrm{p}=0.036)$, and after $24 \mathrm{~h}$, there was still a significant difference between two groups $(\mathrm{p}<0.05)$.

Conclusion: Remote ischemic preconditioning reduces ischemic biomarkers during CABG and attenuates procedurerelated cardiac troponin I release and eventually reduces cardiovascular events such as myocardial infarction, chest pain and hemodynamic changes after cardiac surgery.

\section{Comparison of invasive and} noninvasive blood pressure measurement in patient's candidate to coronary artery bypass graft under cardiopulmonary bypass

\author{
Karami N. ${ }^{1}$, Hassani E. ${ }^{1}$ \\ ${ }^{1}$ Urmia University Of Medical Sciences, Department Of Anesthesiology, Urmia, Iran
}

\section{Abstract}

Backgrounds: Arterial pressure monitoring is one of the standard monitoring in operating room. During general anesthesia blood pressure can be measured by the using a continuous noninvasive arterial pressure method or continuous invasive arterial pressure. Agreement with invasive measurements in patient's candidate to Cardiac surgery has not been assessed. The aim of this study was to comparison of invasive and noninvasive blood pressure in these patients.

Material and method: In a prospective observational study, 38 patients candidate to cardiac surgery under general anesthesia were evaluated. The patients had American Society of Anesthesiologists (ASA) physical status II or III patients and who had complete heart block and marked arterial pressure differences greater than $10 \mathrm{mmHg}$ in the two arms were excluded. Before induction of anesthesia the radial artery is cannulated and noninvasive blood pressure is measured by cuff from other hand at the four time intervals: after radial artery cannulation (To), after induction of anesthesia (T1), before cardiopulmonary bypass (T2), after separation from cardiopulmonary bypass (T3), and at the end of operation (T4).

Result: At the all-time intervals there were no statistically differences between systolic and diastolic blood pressure measurement regarding invasive and noninvasive blood pressure.

Conclusion: According to finding of this study the two method of blood pressure measurement can be used safely in selected patients.

\section{Evaluation of the tracheal tube cuff pressure changes during cardiac operations under cardiopulmonary bypass}

\author{
Mahoori A. ${ }^{1}$, Karami N. ${ }^{1}$ \\ ${ }^{1}$ Urmia University Of Medical Sciences, Department Of Anesthesiology, Urmia, Iran
}

\section{Abstract}

Backgrounds: Tracheal intubation is used for most operations under general anesthesia. Prolonged hyperinflation of the tube cuff can compromise tracheal mucosal perfusion. The aim of this study was the evaluation of changes in intracuff pressure in patients undergoing cardiac surgeries under cardiopulmonary bypass.

Material and method: In a randomized clinical trial 40 patient's candidate to cardiac operations under cardiopulmonary bypass were enrolled. After induction of anesthesia and tracheal intubation by same tracheal tubes, tracheal tube cuff pressure was adjusted to20-25 $\mathrm{mmHg}$ (T0). Then the cuff pressure was measured at beginning of CPB (T1), at $30^{\circ}$ hypothermia (T2) and after separation from CPB (T3)

Result: The mean cuff pressure was 33.5 $\pm 7.3,28.9 \pm 5.4,25.6 \pm 5.2$ and $28.1 \pm 3$ at T0, T1, T2 and T3 respectively.Intra- cuff pressure changed significantly during cardiopulmonary bypass. $(\mathrm{p}=0.0001)$

Conclusion: The mean intra-cuff pressure was decreased during hypotermic cardiopulmonary bypass. The decrease in ICP may protect the tracheal mucosa against hypotensive ischemic injury in these patients. 


\section{-Comparison of acute normovolemic hemodilution effect on the amount of bleeding in the first 48 hours after coronary artery bypass grafting}

\author{
Hasanpour Dargah M. ${ }^{1}$ \\ ${ }^{1}$ Ardabil University Of Medical Science, Anesthesiology, Ardabil, Iran
}

\section{Abstract}

Background: Cardiovascular disease is one of the most common causes of mortality in developed countries, as well as in the whole world. In this regard, autologous transfusion is a topic that can be useful and valuable, especially in complex surgery such as heart surgery and organ transplantation. One of its variants is Acute Normovolomic Hemodilution (ANH). Therefore, the aim of this study was to compare the effect of ANH on the amount of bleeding in the first 48 hours after coronary artery bypass grafting.

Methods: In this clinical trial, 100 patients were selected from all heart patients referred to Imam Khomeini Hospital in Ardebil for CABG surgery in the years 2016-17. They were selected by simple random sampling as a statistical sample and They were divided into intervention (50 ANH recipients' people) and control groups (50 without ANH people).

Results: Based on the findings, in the ANH recipients' group, the mean of bleeding volume was $59.1 \pm 7.3 \mathrm{ml}$ in the first day and $55.6 \pm 4.2 \mathrm{ml}$ in the control group and the difference between the two groups was not significant on the first day. The mean of bleeding volume on the second day was $46.1 \pm 2.8 \mathrm{ml}$ in the ANH group and $42.7 \pm 2.9 \mathrm{ml}$ in the control group. Although it was somewhat higher, it was not statistically significant. Of all samples $45 \%$ were female and 55\% were male. The most common type of blood group was 33 (33\%) in the blood group O. The highest age group (61\%) was over the 60 years old. The pump time varied with an average of $122.2 \pm 21.5$ minutes. The lowest value was PT 11 and the highest was 15 with an average of $13.02 \pm 0.9$ seconds. The mean of PTT was $32.5 \pm 2.6$ seconds and the mean INR was 1.1 \pm 1.0 . The average plt was

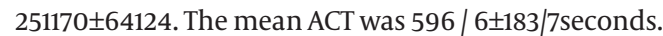

Conclusions: The results showed that in the mean of bleeding volume between the intervention and control groups $(\mathrm{p}=0.41)$ bleeding volume by age $(\mathrm{p}=0.3)$, bleeding volume by gender ( $\mathrm{p}$ $=0.54)$ and bleeding volume by blood group $(\mathrm{p}=0.48)$ was not significant difference. Based on these results, it is suggested that more studies be done on the more number of samples.

\section{- New cardiology clinic conformation new horizon of research and patient care}

Kojuri J. ${ }^{1}$, Kargar S. ${ }^{1}$, Rahmani M. ${ }^{1}$, Gholchinvafa R.', Ahmadi A., Mehdizade M., Heydaralizade R., Zamiri B. ${ }^{1}$, Fatemian $\mathrm{H}_{.}{ }^{1}$, Akbari M.

${ }^{1}$ Shiraz University Of Medical Sciences, Cardiology, Shiraz, Iran

${ }^{2}$ Shiraz University Of Medical Sciences, Cardiology, Shiraz, Iran

\section{Abstract}

Backgrounds: Clinics are not improved with new pace and rev- olution of technology, and generally in majority of clinics there is no availability of patient's data even for legal issues.

In this presentation new clinic structure and software especially designed for cardiology clinics will be introduced that can be rich source of research and excellence of patients care

Material and method: New digital clinics need online appointment service to not only give patients its appointment but can also be a web page for charging and preventing waste of patient's time.

Beside in digital clinic, all data should be collected digitally and history, physical exam, medications, echocardiography, angiography, laboratory data and other workups like exercise tests and holters, should be structurally be recorded in clinic server.

These data can be easily retrievable and be used for reaching to patient data for its care and also for research and especially for any legal issues.

The most important issue is that it can be used and controlled by physician with limited knowledge of data mastery and computer. We invent a single page dashboard cardiology clinic software for data gathering with purpose of data retrieval for research and patient care, in which all medications and laboratory requests can easily be printed in insurance booklets.

Result: We implemented this software since 6 months ago in our clinic and since then more than 4250 patient's data were gathered and 2 research were undergoing, beside more than 100 students were participating in patient data registry and research and clinic patient management.

All students were satisfied with format of registry with satisfaction rate of more than $97 \%$ and rate of patient's satisfaction was more than $88 \%$.

Conclusion: New cardiology clinic design is different and is a rich source of research and excellence of patient care.

\section{- The inferior vena cava stent in a child with Budd-Chiari syndrome A case report}

\section{Mortezaeian H.', Firouzi A. ${ }^{1}$, Jafari F.}

${ }^{1}$ Rajaie Cardiovascular Medical And Research Center, Interventional Cardiology, Tehran, Iran

\begin{abstract}
Backgrounds: Budd-Chiari syndrome (BCS) defined as hepatic vein outflow tract obstruction located in hepatic veins or inferior vena cava (IVC) or both of them. The prevalence of BCS is about 2.40-33.10 per million. One of the causes of BCS is membranous obstruction of the inferior vena cava.

Material and method: This study reports a 9 years old boy with a history of abdominal pain, nausea, and fatigue. In physical examination, he had hepatomegaly and hydrocele. Abdominal sonography showed thrombosis in hepatic veins and suprahepatic portion of IVC. Echocardiography showed normal cardiac function and thrombosis in IVC. In imaging reports, no evidence of an abdominal mass or compression effect on IVC was depicted. Bone marrow aspiration and hematologic evaluation were normal. Treatment with enoxaparin and hydroxyurea was done for 3 weeks with no effect on the size of IVC thrombosis. In MR venography with Gadolinium filling defect and severe stricture of intrahepatic to supra hepatic portion of IVC was depicted.

Result: In angiography, IVC injection showed interrupted IVC. At first, the wire pass from IVC to PA and then balloon angioplasty was performed with power flex $10 / 30 \mathrm{~mm}$. IVC stent was done
\end{abstract}


with CP stent $39 \mathrm{~mm}$ and BIB balloon 14/40 mm successfully. The patient discharged 2 days after the procedure with Aspirin and Clopidogrel. 2 weeks later follow up showed patent IVC stent in Doppler sonography and Echocardiography.

Conclusion: Percutaneous stent angioplasty can be considered the first-line management for BCS patients with membranous obstruction of IVC because of its minimal-invasive method.

Keywords: Budd-Chiari syndrome, stent

\section{An attractive case of a vascular complication in a young man}

\author{
Firouzi A. ${ }^{1}$, Aminbeidokhty A. ${ }^{1}$, Norouzi Z. ${ }^{2}$ \\ ${ }^{1}$ Rajaie Cardiovascular Medical And Research Center, Interventional Cardiology, Teh- \\ ran, Iran \\ ${ }^{2}$ Rajaie Cardiovascular Medical And Research Center, Cardiac Rehabilitation, Tehran, \\ Iran
}

\section{Abstract}

Backgrounds: A 21-year-old male was presented with a history of intermittent chest discomfort and a low-grade fever for 2 months. He had a history of a lower limb pain for 6 months. Also, he mentioned a history of a car accident and a trauma to his chest three years earlier without any serious injury at that time. In the physical exam, a systolic $2 / 6$ murmur in the aortic area was heard. Also, the upper limbs pulses were stronger in comparison with the lower limbs.

Material and method: ECG (electrocardiogram) revealed sinus tachycardia.Laboratory tests including: $\mathrm{Hb}$ (Hemoglobin): $12.4 \mathrm{~g}$ / dL, WBC (White Blood Cells): 10500 cell $/ \mathrm{mm}^{3}$, ESR (Erythrocyte Sedimentation Rate): $110 \mathrm{~mm} / \mathrm{h}, \mathrm{CRP}$ (C-Reactive protein): 10.3 $\mathrm{mg} / \mathrm{L}$ and Calcium: $8.4 \mathrm{mg} / \mathrm{dL}$

On echocardiography, LVEF (Left Ventricle Ejection Fraction) was 55\%. A web-like narrowing adjacent to LSCA (Left Subclavian Artery) with a pressure gradient of $60 \mathrm{mmHg}$ with diastolic tail and holo antegrade diastolic flow in abdominal aorta was seen that was compatible with aortic coarctation.

PET CT (Positron Emission Tomography/ Computed Tomography) scan was done and there was no evidence of aortitis, so active vasculitis was rolled out.

Result: In the end, with the diagnosis of aortic pseudoaneurysm, the patient underwent TEVAR (Thoracic Endovascular Aortic Repair) and all of the signs and symptoms were disappeared. In fact, the neglected chest trauma was the leading cause of gradually pseudoaneurysm formation during three years.

Conclusion: Paying more attention to the chest trauma and following the patients, plays an important role in preventing the traumatic delayed vascular complications. Close follow up and serial imaging if needed, in every patient with chest trauma is recommended.

\section{Percardial decompression syndrome}

\section{Bayanati M. ${ }^{1}$}

${ }^{1}$ Madaen Hospital, Cardiology, Tehran, Iran

\section{Abstract}

Backgrounds: Pericardial tamponade is a complication of percutaneous treatment of chronic coronary artery occlusions. Per- icardial decompression syndrome is a rare but potentially fatal complication of surgical or percutaneous drainage of pericardial fluid. The case presented here is a case of pericardial tamponade following percutaneous treatment of right coronary artery chronic total occlusion in which hemodynamic condition after pericardiosynthesis deteriorated due to severe right ventricular enlargement and dysfunctionan.

Material and method: A 73 years old lady underwent percutaneous treatment of right coronary artery chronic total occlusion. She developed pericardial tamponade and hypotension 6 hours later and pericardiosynthesis was done. Soon after pericardiosynthesis hemodynamic deterioration ensued due to severe right ventricular enlargement and dysfunction. The patient underwent conservative management using intravenous fluid and inotropic support. Right ventricle recovered 12 hours later and patient discharged with good general condition on the third day.

Result: Cardiogenic shock and severe right ventricular enlargement and dysfunction in the presented case was most likely due to pericardial decompression syndrome. Conservative treatment was efficient and effective.

Conclusion: Pericardial decompression syndrome is a rare complication of surgical and percutaneous treatment of pericardial tamponade and can be treated conservatively.

\section{The role of vitamin $D$ on the left ventricle ejection fraction in thalassemia major patients with cardiac hemosiderosis in children's hospital Tabriz 1395}

\author{
Hiradfar A. ${ }^{1}$, Jamei Khosroshahi A. ${ }^{2}$, \\ Hassanzadeh A. $^{3}$ \\ ${ }^{1}$ Children'S Hospital, Oncology Department, Tabriz, Iran \\ ${ }^{2}$ Children'S Hospital, Pediatics Cardiology Department, Tabriz, Iran \\ ${ }^{3}$ Children'S Hospital, Pediatric Department, Tabriz, Iran
}

\section{Abstract}

Backgrounds: Due to vitamin D insufficiency in population, this problem could be observed in thalassemia patients because of increased metabolic needs and higher iron storage. Vitamin D insufficiency in these patients and its relation to leftventricular ejection fraction (LVEF), left ventricular dysfunction and cardiac iron overload has been investigated in many studies. The very purpose of this study was to investigate efficacy of treating with high dose vitamin D in improving LVEF in thalassemia patients with heart failure and vitamin $\mathrm{D}$ deficiency.

Material and method: This clinical trial study was conducted on 29 chronically transfused thalassemiapatients and ventricular dysfunction with vitamin D deficiency in 1395, in Thalassemiaclinic, Tabriz Children's Hospital. Mean age of the patients was $20.35 \pm 4.91$ years.A serum 25-hydroxy vitamin D3 level less than 30ng/dl was considered vitamin D deficiency in thisstudy. LVEF less than $55 \%$ was indicated as poor pump function. Patients received 50,000 IU of vitaminD3 weekly, for 8 weeks. Data on LVEF, electrolytes and serum vitamin D were compared during 6 months of study.

Result: Means of serum 25-OHD3 levels, before and after the study, were $13.10 \pm 5.91 \mathrm{ng} / \mathrm{ml}$ and $64.04 \pm 10.39 \mathrm{ng} / \mathrm{ml}$, respectively ( $p=0.01)$. Means of LVEF were $30.38 \pm 12.82 \%$ and $49.61 \pm 9.23 \%$ before and afterthe study, respectively $(\mathrm{p}=0.03)$. Means of serum ferritin 
levels and cardiac T2* score of the patients were $3913.06 \pm 2229.34$ $\mathrm{ng} / \mathrm{ml}$ and $1.93 \pm 0.96$, respectively.Serum parathyroid hormone (PTH) levels of the patients at the beginning of the study and at the end of 2 ndmonth were $94.20 \pm 18.55$ and $43.65 \pm 17.31 \mathrm{ng} / \mathrm{ml}$, respectively $(\mathrm{p}=0.03)$. There was a positive correlation between mean of serum 25-OHD3 level and cardiac MRI T2* parameter at the beginning of the study $(\mathrm{r}=0.001)$. There was a positive correlation between mean serum 25-OHD3 and LVEFat the end of study $(\mathrm{r}=0.001)$.

Conclusion: Results showed that vitamin D3 was effective and safe in improving LVEF and cardiacdysfunction in transfusiondependent thalassemia patients with vitamin D deficiency.

\section{Delayed induced heart failure in burn injury}

\author{
Harati H. ${ }^{1}$, Momeni M. ${ }^{1}$, Amrollahi S. ${ }^{1}$ \\ ${ }^{1}$ Shahid Motahari Burn Hospital, Icu, Tehran, Iran
}

\section{Abstract}

Backgrounds: Heart failure is established as a cause of increasing morbidity and mortality post massive burn injury. Beginning immediately in the ebb phase, cardiac function is severely decreased, by 48 h post injury cardiac function rebounds and the post burn myocardium becomes tachycardic and hyperinflammatory. Although some of severely burnd patients which was asympthomatic in the first days developed cardiovascular failure after a period of 2-3 weeks of the initial injury. This delay in the presentation of heart failure may be due to different mechanisms that should be considered.

Material and method: 6 of our patients aged(18-29 years old 5 men, one woman) with more than 70\% burn in ICU developed dyspnea, pulmonary congestion and cardiomegaly in $\mathrm{x}$ ray after an asymptomatic period of 2-3 weeks. Tachycardia was present and in echocardiography LVEF was moderate to severely decreased( $30-40 \%$ ) with global hypokinesia .RV size and function was normal and PAP mildly increased.

Procalcitonin was low and mean hemoglobin was 9(12-8.2), renal function and GFR was in the normal range in all of them. One of the patients has a wide pulse pressure and increased in RBC volume in $\mathrm{CBC}$ with a good respond to vitamin B supplements. All the six received conventional heart failure treatment of diuretics, betablockers( carvedilol ), ACEI, and spirinolacton, and five of them required ionotropic support .

Result: Four passed the acute phase of failure and came out of congestion, Three of them survived( one with vitamin B deficiency). One died of sepsis.

Conclusion: Severe burn injury is devastating state and cardiac dysfunction in this situation increase motality careful examination and early detection of nutritional deficiencies that may present after a prolonged ICU care and lead to heart failure in these patients may improve outcome.

\section{Percutaneous Pulmonary Artery Balloon valvotomy}

\section{Ghaderian M. ${ }^{1}$, Ahmadi A. ${ }^{1}$}

${ }^{1}$ Pediatric Cardiovascular Research Center Cardiovascular Research Institute Isfahan University Of Medical Sciences, Pediatric Cardiology, Isfahan, Iran

\section{$\underline{\underline{\text { Abstract }}}$}

Backgrounds: Pulmonary valve atresia with intact ventricular septum is one of the rare diseases in neonates that is dependent on the patent ductus arteriosus (PDA) and is an emergency of congenital heart disease. The purpose of this study is to introduce two cases of percutaneous balloon valvotomy of the polmunary artery valve.

Material and method: In this study, two neonates that referred to Shahid Chamran center affiliated to Isfahan University of Medical Sciences at the beginning of the birth with pulmonary atresia was studied. In these two patients, we used femoral and arterial approaches and entered pulmonary arteries via PDA and after crossing the guide wire from pulmonary valve, different sizes balloons passed over the guide wires up to maximum size and then the blood flow and the pressure were measured (figure-1 A,B). Result: Two neonates aged 2 days and weighed $2800 \mathrm{~g}$ and 3100 $\mathrm{g}$ respectively were included in this study. The pulmonary artery pressure in these patients was 18 and $15 \mathrm{~mm} \mathrm{Hg}$, and right ventricular pressure was 120 and $110 \mathrm{~mm} \mathrm{Hg}$. The systemic O2 saturation were $65 \%$ and $70 \%$, respectively. One of the patients had severe tricuspid regurgitation. The right ventricular pressure in these two patients dropped to about $30 \mathrm{mmHg}$ after the procedure. An increase in pulmonary pressure of about $5 \mathrm{~mm}$ was observed in these two neonates. General condition of the patient who had severe tricuspid regurgitation improved slightly over a week after the procedure. Increasing of $\mathrm{O} 2$ sat after the procedure in both patients was seen that had faster in first patient. Conclusion: Pulmonary artery balloon valvotomy in PDA dependent patients and low $\mathrm{O} 2$ sat is an important and urgent procedure that plays an essential role in improving the quality of life in these patients.

\section{Aspirin response monitoring by impedance aggregometry in children with systemic-to-pulmonary shunts or PDA stent}

\author{
Malekan Rad E. ${ }^{4}$, Dehestani A. ${ }^{5}$ \\ ${ }_{1}^{1}$ Tehran University of Medical Sciences Iran, Tehran
${ }^{2}$ Tehran University of Medical Sciences Iran, Tehran
${ }^{3}$ Tehran University of Medical Sciences Iran, Tehran
${ }^{4}$ Tehran University of Medical Sciences Iran, Tehran
${ }^{5}$ Tehran University of Medical Sciences Iran, Tehran
}

Faghihi T. ${ }^{1}$, Sayadpour Zanjani K. ${ }^{2}$, Torkan N. ${ }^{3}$,

\section{Abstract}

Backgrounds: Shunt thrombosis after implantation of systemic-to-pulmonary shunts in pediatric patients is common. Aspirin effect is rarely monitored, although it is known that the response varies. The aim of the present study was to determine the effects of acetyl salicylic acid medication on platelet aggregation in children with systemic-to-pulmonary shunts.

Material and method: In a prospective study, children who were treated with aspirin after shunt implantation or PDA stenting were included. Platelet aggregation in whole blood was analyzed with impedance aggregometry at least 7 days after aspirin administration. Echocardiography test was carried out at two time points (at least 7 days after aspirin administration and 3 months after the first observation). All patients were evalu- 
ated for shunt thrombosis by echo and minor/major bleeding as well as symptomatic clinical thrombosis in that time period. We aimed to double the dose of aspirin in patients who demonstrated aspirin resistance based on impedance aggregometry results. Result: Aspirin reduced the arachidonic acid-induced platelet aggregation in all of the patients. All participants were in the therapeutic range in 7 days after aspirin intake. No cases of major bleeding were observed. No cases of clinical and para-clinical thrombosis were be detected.

Conclusion: Aspirin reduces platelet aggregation after shunt implantation and arterial stenting in pediatric patients. Further trials are needed to elucidate role of monitoring aspirin therapy by impedance aggregometry in children with shunts and PDA stent.

\section{- Enteral Nutritional Support by Nurses and Related Factors in Intensive Care Patients}

\section{Babapour S. ${ }^{1}$, Esmaeili R. ${ }^{2}$, Esteki T. ${ }^{3}$, Pourhoseingholi M. ${ }^{4}$}

${ }^{1}$ Imam Khomaini Hospital, Master Of Critical Care Nursing, Freidonkenar Mazandaran, Iran

${ }^{2}$ School Of Nursing Midwifery Shahid Beheshti University Of Medical Sciences, Assistant Professor Of Nursing, Tehran, Iran

${ }^{3}$ School Of Nursing Midwifery Shahid Beheshti University Of Medical Sciences, Assistant Professor Of Nutrition And Nursing, Tehran, Iran

${ }^{4}$ Research Institute For Gastroenterology And Liver Diseases Shahid Beheshti University Of Medical Sciences, Associate Professor Of Biostatistics, Tehran, Iran

\section{Abstract}

Backgrounds: Enteral nutritional support is vital to improve clinical outcomes in critical patients, but there are many challenges in its provision. Nurses have an important position and play a key role in nutritional support.The purpose was to determine the enteral nutrition support by nurses and related factors in intensive care units (ICUs) patients during the first 3 days after initiation.

Material and method: This descriptive-correlation study, the amount of energy and protein received and required by 48 ICUs patients calculated using Harris-Benedict equation and the American Dietetic Association guidelines. Enteral nutrition performance of 100 nurses in ICUs evaluated using checklist. Then the nurses completed questionnaire about related factors to nutritional support. The content validity index was 0.93 and 0.95 in the checklist and questionnaire, respectively. The reliability of checklist and questionnaire using intra-class correlation coefficient (ICC) and Cronbach's alpha coefficient were 0.89 and 0.83 respectively.

Result: The results indicated energy and protein received were less than required on each of the three days. The nurses' performance score in enteral nutrition was moderate (57.49 \pm 9.58) and the lowest score associated with the questionnaire items were, the dietitian daily presence (28.5\%) and on holidays $(8.75 \%)$, the existence of guideline (34\%). The most common causes of interruptions reported gastrointestinal intolerance (77.5\%).

Conclusion: It seems that planned interventions are required for overcoming all identified problems. The constant presence of a nutritionist in ICU's team and the presence of appropriate guidelines together with training on proper implementation is necessary for improvement of enteral nutrition.

\section{Internet of Medical Things IOMT Applications and Challenges}

\author{
Nami M. ${ }^{1}$, Saneipour Z. ${ }^{2}$ \\ ${ }^{1}$ Qiau, Electrical It And Computer Engineering, Qazvin, Iran \\ ${ }^{2}$ Aja University, Besat Hospital, Tehran, Iran
}

\section{Abstract}

Backgrounds: It is anticipated that the demand for personal healthcare applications will increase sharply. In the traditional medical mode, the quality and scale of medical service can't meet the needs of patients.As IOT converge various domains, we focus on research contribution of IOT in healthcare domain and describe Internet of Medical Things (IOMT) as a solution. IOMT is playing vital role in healthcare industry to increase the accuracy, reliability and productivity of electronic devices. Researchers are contributing towards a digitized healthcare system by interconnecting the available medical resources and healthcare services. This paper presents IOMT concepts including definitions,characteristic, issues and major challenges.

Material and method: Basically IOT is the inter-networking of electronic devices to enable exchange of data between devices for specific domain applications.It is of great significance to establish a set of family oriented remote medical surveillance system based on mobile Internet ascalled $\mathrm{m}$ - health, which is used to analyze, capture, transmit and store health statistics from multiple resources, including sensors and other biomedical acquisition systems. M-health offers an elegant solution to a problem commonly faced in the medical field: how to access the right information when and where needed in highly dynamic and distributed healthcare organizations. There are some applications in medical domain such asSmart medical nursing healthcare system for patients andIot based smart medical health band implemented and delivered in 2016. In IOT applications, the patient healthcare information is the most critical information that should be kept in safe hands. Iot has bought a tremendous change in the domain of medical.Most of the applications are from the research papers which are published in 2016. So, our researched applications are up to date.

Result: IOT has many advantages to individuals, society, the environment, consumers and business.IoT enable the doctors and hospital staff to do their work more precisely and actively with less effort and intelligence. Proof of this is above mentioned table II of applications. Some of IOMT benefits are describes as follows: Make life more convenient Healthcare is cheap Outcome of patient is improved Management of diseases is real-time Life quality is Improved user end experience is improved care for patient is increased costs reduction Ultimate benefit is healthier and longer lives, Maximum diseases management and prevention children's / elder parents progress is monitored Major change in health of patient will make an automatic alert to different parties, save lives and time Resources of iot other iot devices,Medication is on time Patient care will be intimated to family members.

Conclusion: We havediscussed mainly the applications, future challenges and benefits of internet of things (IOT) based on the work done by different researchers in the field of IOT. All the applications we researched are from the medical healthcare systems. Most of the applications are from the research papers which are published in 2016. Actually there are many challenges that has to be counter but we have briefly identified some of the significant challenges in the file of IOT in context of healthcare that are detailed discussed in section III. We believe that if these challenges are met in the field of IOT, we can improve IOT stand- 
ard in the field of medical care. IOT can provide more reliable and better services in the field of medical health care.

\section{Association between fear of falling and functional dependency in heart failure patients in 2018 Tehran-Iran}

\section{Beikverdi M. ${ }^{1}$}

${ }^{1}$ Tehran University, Nursing Faculty, Tehran, Iran

\begin{abstract}
Backgrounds: Fear of falling is a common restricting factor for functional dependency especial amongst older adults. This study aimed to determinate factors associated with fear of falling and functional independency in older adults with Heart failure.

Material and method: This was a cross-sectional study using a random sampling method to enroll 445 patients between September 2017 and November 2018. Main outcome measurement instruments were the 16- item falls efficacy scale international (FES-I, Persian version) and Ketz index (KI) functional independence measure. Variables In this study were analyzed by Student $t$ test and ANOVA. The Pearson coefficient correlation was used to examine the relationship between FES and functional independency. A 2-tailed $\mathrm{P}<0.05$ was considered statistically significant.

Result: The patients were mostly male (66\%) and aged about 70.6. 60.8\% of all participants experienced one or more falls during the last year. There was a significant relationships between the fear of falling and functional independence $(r=-0.257 ; \mathrm{P} \leq$ $0.001)$. But between fear of falling and demographic characteristic there wasn't a significant relationship $(P>0.05)$

Conclusion: According to the findings, there was a median leve of fear of falling in most of the patients. The more fear of falling the more functional dependency.
\end{abstract}

\section{- Criterions of predicting veins thromboembolism}

\section{Abbasi Dolatabadi Z. ${ }^{1}$, Sayadmansor M. ${ }^{2}$, Zarei Mahmoodabadi A. ${ }^{3}$}

${ }^{1}$ Phd Professor Assistant Faculty Of Nursing Midwifery Of Tehran University Of Medical Sciences, Faculty Of Nursing Midwifery Of Tehran University Of Medical Sciences, Tehran, Iran

${ }^{2}$ Emam Khomeini Hospital, Emergency Ward, Tehran, Iran

${ }^{3}$ Faculty Of Nursing Midwifery Of Tehran University Of Medical Sciences, Faculty Of Nursing Midwifery Of Tehran University Of Medical Sciences, Tehran, Iran

\footnotetext{
Abstract

Backgrounds: Thrombosis of the deep veins could be counted as a reason which increase the rate of death among the cardiovascular patients who have been hospitalized in special care sections. Early diagnosis and treatment could prevent the consequences of this disorder. The present study have been conducted for the purpose of studying the criterions of predicting the veins thromboembolism including lung embolism and thrombosis of the deep veins Material and method: In this review study, databases such as Science direct, Ovid, Scopus, Up to Date. SID, and Magiran was used to data collection. Articles published between 2000 to 2018 end of March.
}

Findings:Early diagnosis of thromboembolism can help the on time treatment and reduce the long term side effects as well as early death who are suffering from lung thromboembolism. Therefore different criterions such as VELZ, TERYARD, and VIRSHO can be used.

Result: With the help of different criterions, nurses of the special care sections can diagnose the high risk patients who may be exposed to the vein thromboembolism. Also nurses could play an important role in presenting the special care and preventing the side effects of hospitalization as well.

Conclusion: Nurses in intensive care units have an important role in reducing the length of hospitalization, reducing hospital infections, controlling the complications of admission in special sectors, and reducing the number of hospitalization in health care centers, especially in intensive care units. Improving the ability of nurses improves their clinical competence. Therefore, it is necessary to continuously familiarize with the world's standards and standards in the field of prevention, diagnosis and care, and to pay attention to the various issues of caring for patients and patients in continuing education of nurses.

\section{Processes underlying concordance to treatment regimen A grounded theory study}

\author{
Vahedparast H. ${ }^{1}$, Mohammadi E. ${ }^{2}$, Ahmadi F. ${ }^{2}$ \\ ${ }^{1}$ Bushehr University Of Medical Sciences, Nursing, Bushehr, Iran \\ ${ }^{2}$ Tarbiat Modares University Tehran, Nursing, Tehran, Iran
}

\section{Abstract}

Backgrounds: Patients concordance to treatment regimen is the one of the main challenges of treating common chronic diseases including coronary artery disease (CAD) and diabetes. This is a complex process and influenced by many factors especially underlying cultural, social and economic context of the country. Aim: explore how patients with CAD and diabetes concordance to treatment regimen.

Material and method: Methods: a qualitative research based on grounded theory was carrying out with purposeful data collection. Then it was theoretically continued from homes, hospitals and clinics. A total 46 participants entered the study. Data was collected through unstructured individual interview and field notes, and analyzed simultaneously with Strauss and Corbin's methods.

Result: Results: constant comparative analyses of data showed the patients concordance to treatment regimen is based on emergency self-preservation behavior in response to fear of dealing with risk, which is the main concern of patients with CAD and diabetes. Emergency self-preservation develops in the context of inadequate concordance to treatment regimen. Conflicting priorities and perceived needs, limiting induction \& beliefs (Constraining Factors) and perceived social support (as factors facilitating) are some of the factors affecting this process. The ultimate outcome of adopting such strategy and the affecting factors is the insecurity and the failure to accept the modified behaviors.

Conclusion: the finding show that emergency self-preservation as the manner of chronic patients concordance to treatment regimen is proportionate to their problem and concern but nevertheless undesirable (emergency based and transient), inefficient and ineffective (insecurity and failure to accept the initial treatments). 


\section{Early extubation after congenital heart surgery narrative review}

\author{
Eskandari S. ${ }^{1}$, Jalaly P. ${ }^{2}$ \\ ${ }^{1}$ Shahid Beheshti University Of Medical Sciences, Nursing And Midwifery Faculty, Teh- \\ ran, Iran \\ ${ }^{2}$ Tabriz Branch Islamic Azad University, Medical School, Tabriz, Iran
}

\begin{abstract}
Backgrounds: mechanical ventilation is used widely in the management of patient following open heart surgery and advances in anesthesia and surgical technique have altered the expectation for mechanical ventilation following cardiac surgery the purpose of this review study is to assess the adequacy of early extubation after congenital cardiac surgery

Material and method: This study was conducted through a narrative review and after searching in reputable scientific bases such as Pubmed, Scopus and Google Scholar. After researching and collecting articles based on input and output criteria,Five papers that were sufficiently valid were selected for review. Result: a study in 2000 showed that of 201 congenital open heart surgery cases, 175 (87.1\%) were extubated in the OR while 188 (93.6\%) were extubated within four hours of the operation. In 2008, Mittnacht and colleagues reported that OR extubation was successful in $79 \%$ of their pediatric patients (178/224), even in cases where complex procedures were involved. They concluded that the greatest risk factors were young age and a longer duration of cardiopulmonary bypass .In 2014, Grag and colleagues reported that OR extubation was successful in $87.1 \%$ of their cases with success rate dependent on the details of the procedures performed. Conclusion: Extubation in the OR after congenital open-heart surgery was successful in the majority of our patients, even following complex procedures. However,longer operation time is a significant risk factor with regards to re-intubation in the ICU after early extubation.
\end{abstract}

\section{An evaluation of the effectiveness} of social media-based training on satisfaction and anxiety of families of patients who are under Coronary artery bypass surgery at Intensive Care Unit ICU

\author{
Ershad S. ${ }^{1}$ \\ ${ }^{1}$ Rajae Hospital, Nursing, Tehran, Iran
}

\begin{abstract}
Backgrounds: Families patient as result of receiving insufficient information go under immense anxiety before the surgery and during hospitalization. Providing information and training them via social media help in reduced anxiety and increased satisfaction . Material and method: This research was a quasi-experimental study which allocated to intervention and control groups that data collection has done on 100 cases of Families patient has gone under Coronary artery bypass surgery on 2017 which were selected by convenience sampling method, in Rajaie cardiovascular medical .The intervention group became members of a Telegram network this group received the information regarding the surgery and post-surgery treatments through this channel. For the control group, however, only routine actions were taken.
\end{abstract}

For data collection, Spielberger's State-Trait Anxiety Inventory (STAI) questionnaire and Intensive Care Unit Family Satisfaction questionnaire was used. Data's were analyzed in SPSS.

Result: Based on the findings, there was no statistically significant difference between the two groups before the intervention. But after the intervention, the average number for the State-Trait Anxiety significantly dropped and satisfaction following receiving information significantly increased in, intervention group as compared to the control group. $(\mathrm{p}<0 / 0001)$

Conclusion: Based on findings, social media-based training leads to increased patients family satisfaction and decreased anxiety in Intensive Cardiac Care Unit.

\section{- Validity and Reliability of the professional communication skills of nurses Questionnaire}

\section{Tamimi T. ${ }^{1}$}

${ }^{1}$ Bushehr University Of Medical Sciences, Department Of Anesthesiology Faculty Of Paramedicine, Bushehr, Iran

\section{Abstract}

Backgrounds: The professional obligation of nurses and their skill may importantly impact on communication through enhancement of secure care of patient, reducing stresses in patients' attendants, decreasing stress among the colleagues. Given that identifying of care requirements for patients and meeting them is the main task for the nurses thus improvement of behavioral and communication skills will be efficient in achieving this objective in nurses. Similarly, observance of the principles of proper communications among members of medical team is considered as one of the foremost factors that may play key role in giving suitable services to the patient as a human to release him/her from illnesses for which she has allowed medical team to enter into his/her privacy.The purpose of this study was to develop an instrument to measurethe professional communication skillsin nurses.

Material and method: In the present cross-sectional study, the initial list of 32 questions was prepared to design a genuine questionnaire of awareness of professionalcommunication skillsin nursing. In order to check the the content validity and reliability, the Cronbach's Alphaand Kappa Coefficienttest was used. Data were analyzed using the SPSS software version 21.

Result: The results indicated that just 25 question from 32 initial list had high content validity. The final results disclosed that the final questionnaire was reliable with a Cronbach's alpha of 0.89 . Conclusion: To assess the professionalcommunication skillsin nursing, a reliable questionnaire should be used. The questionnaire designed in the present study may be a useful tool for evaluation of it.

keyword: professionalcommunication,validity, reliability.

Evaluation of the Critical thinking in the nurses who work at the Intensive Care Units in hospitals of Universities of Medical Sciences 
${ }^{1}$ School of Nursing Research Center For Nursing Midwifery Care Shahid Sadoughi University Of Medical Sciences Yazd Iran, Nursing, Yazd, Iran

\begin{abstract}
Backgrounds: Critical thinking skill is one of the most important goals of nursing education and its development leads to improve the patients' treatment. Due to the importance of this issue in improving the quality of nursing cares especially in the Intensive Care Units (ICUs), so the present study was conducted aimed to evaluate the critical thinking in ICUs

Material and method: This study is a cross-sectional one. California Critical Thinking Skills Questionnaire B was completed for nurses who work at ICUs of hospital. The data was analyzed by SPSS software version 22

Result: The average of critical thinking scores of all nurses was $10.46 \pm 3.17$ in this study which was poor. The most ability of nurses was related to the domain of deductive reasoning and the weakest was in the domain of inference. There was no significant difference between critical thinking and its relevant areas in terms of age, sex, marital status, working part, university and work experience $(\mathrm{P}>0.05)$.

Conclusion: The results of this study revealed a low score of critical thinking in nurses who work at ICUs. Due to the importance of this skill in nursing activities, using specific educational strategies is emphasized to improve this skill.
\end{abstract}

\section{Training of ECMO Nurses}

\section{Azizi B. ${ }^{1}$}

${ }^{1}$ Shahid Faghihi Hospital, Cardiac Surgery, Shiraz, Iran

\section{Abstract}

Backgrounds: extracorporeal membrane oxygenation (ECMO) is an extracorporeal technique of providing both cardiac and respiratory support oxygen to patients whose heart and lungs are so severely diseased or damaged that they can no longer serve their functions.

Material and method: ECMO is a high-risk procedure and therapy. An ECMO nurse is a bedside nurse that cares for ECMO patients and has a modified responsibility for assessing and managing the ECMO system.

Result: The nursing team caring for the patient supported on ECMO requires specialist training to ensure safe and effective care. All training must be supported by a robust assessment and regular reassessment. After completion of an initial education program, it is important to provide ongoing continuing education and clinical competence assessment. In order for continuing ECMO education to be successful, the proper infrastructure for continuing education in ECMO is essential

Conclusion: In this article we will introduce a standard nursing training model.

Keywords: ECMO, ecmo nurse, training

\section{Teamwork in nursing of cardiac surgery}

\author{
Safari Soltanabad A. ${ }^{1}$ \\ ${ }^{1}$ Emam Reza Hospital Mashhad, Cardiac Surgery, Mashhad, Iran
}

\section{$\underline{\text { Abstract }}$}

Backgrounds: Teamwork is the collaborative effort of a team to achieve a common goal or to complete a task in the most effective and efficient way. This concept is seen within the greater framework of a team, which is a group of interdependent individuals who work together towards a common goal.

Material and method: Teamwork is present in any context where a group of people are working together to achieve a common goal. Cardiac surgery demands effective teamwork for safe, high-quality care.

Result: Surgical excellence demands teamwork. Poor team behaviors negatively affect team performance and are associated with adverse events and worse outcomes. Interventions to improve surgical teamwork focusing on frontline team members' nontechnical skills have proliferated but shown mixed results. Conclusion: cardiac surgery teams involve in treatment of patient in cardiac surgery procedure. The patient's final prognosis is just as relevant to the team's performance, which is related to the knowledge and skills of each individual. The members of the cardiac surgeryteam, as one of the teams involved in the treatment of patients, must comply with the rules of successful teams to achieve better performance and more success in providing health care to patients. Learning the principles of teamwork in nursing requires, like any other skill, learning, training, and advice.

\section{- Preparation and spiritual Support of Patients and their families through the heart Transplant Process}

\author{
Nasrizadeh Moghaddam S. ${ }^{1}$ \\ ${ }^{1}$ Dena Hospital, Cardiac Surgery, Shiraz, Iran
}

\section{Abstract}

Backgrounds: Cardiac allotransplantation has, over the years, become the established therapeutic modality for patients with end-stage heart failure. Significant advances in immunosuppressive therapy have dramatically improved the outcome of heart transplantation over the past four decades.

Material and method: Although ,Cardiac transplantation is still associated with significant morbidity, mortality, and supportive care needs.

Result: role of nurses in heart transplant surgery is very important. Nursing is in direct contact with the patient, mainly in the preoperative and postoperative heart transplantation, and telling the patient about the procedure doubts, overcoming anxiety and clarifying the family and the transplanted patient ${ }^{\prime} \mathrm{s}$, the necessary lifestyle care changes.

Conclusion: In this article we will explain nursing care plan for this patients especially focousing on nursing education to the patients on the operation process and risks of complications, family's questions and misconceptions about organ transplantation, diets and other long-term lifestyle changes. Keywords: Heart transplant, nursing care

\section{Reducing the anxiety in parents of children with congenital heart anomaly and its effect on the treatment process}

\author{
Jamali M. ${ }^{1}$, Gheysardoost M. ${ }^{1}$ \\ ${ }^{1}$ Dena Hospital, Cardiac Surgery, Shiraz, Iran
}




\section{Abstract \\ Backgrounds: Congenital Heart anomalies are one of the com- mon diseases; based on the American Heart Association esti- mate, 40,000 newborns per year are born with this disease in the United States. \\ Material and method: The parents of these children are con- fronted with great anxiety due to their lack of information about the prognosis and treatment process. \\ Result: during treatment and illness, these children are 100\% dependent on parental support, this anxiety is transmitted to them, which while has a bad effect on the treatment process, is often ignored in general nursing interventions. \\ Conclusion: This study was conducted to improve the treat- ment process through behavioral and cognitive interventions of parents and control and management of stress factors. Obvi- ously, the target groups are both family and patient. \\ Keywords: Congenital heart disease, anxiety, nursing care \\ - Hospitalization In the intensive care unit care of patient or family}

\author{
Salman Dehkordi F. ${ }^{1}$, Imanipour M. ${ }^{2}$ \\ ${ }^{1}$ Msc Student Of Intensive Care, Tehran University Of Medical Sciences, Tehran, Iran \\ ${ }^{2}$ Faculty Member Of Tehran University Of Medical Sciences, Tehran University Of Medi- \\ cal Sciences, Tehran, Iran
}

\section{Abstract}

Backgrounds: In this article, the implications of patient admission in the intensive care unit on family caregivers of patients are reviewed and symptoms and preventive and caring approaches in the intensive care syndrome family is described in detail.

The environment of the intensive care unit (ICU) is burdensome to a patient and the patient's family. Unfortunately, many family members who assume a caregiving role during critical illness experience the detrimental effects of this stressful life event and at risk the cluster of adverse psychological reactions, such as: anxiety, acute stress disorder, posttraumatic stress disorder, depression and complicated grief, The post-intensive care syndromefamily (PICS-F) describes this psychological disorders, occurring in approximately $14 \%$ to $50 \%$ of family members of ICU patients. Material and method: This review study was done in a systematized manner and based on library literatures. To do this, the articles published on family of critical patients during 2005-2018, searched out in different Farsi and English databases using keywords of: intensive care unit, patient's family, Post-intensive care syndrome-family, Anxiety, Depression, and PTSD

Result: Post-intensive care syndrome-family (PICS-F) refers to new or increased cognitive or mental health impairment in family members after a loved one is hospitalized in a critical care setting. each of the treatment team members in intensive care unit (ICU) should support the family members in dealing with the stressful situations.

Conclusion: The social support and attitudes of ICU personnel, particularly good communication, are factors reducing negative psychological consequences among family members.

\section{- Wearable cardioverter-defibrillator for primary prevention of post-myocardial infarction sudden cardiac death}

${ }^{1}$ Gonabad University Of Medical Sciences, Bohlool Hospital, Gonabad, Iran

${ }^{2}$ Isfahan University Of Medical Sciences, Milad Hospital, Isfahan, Iran

\section{Abstract}

Backgrounds: Despite effective interventional and medical therapies for myocardial infarction, the risk of early post-MI sudden cardiac death (SCD) remains, especially in patients with severely reduced left ventricular ejection fraction (LVEF). However, there remain situations in which implantation of an ICD is immediately not feasible (eg, patients with an active infection), may be of uncertain benefit, may not be covered by third-party payers (eg, early post-MI, patients with limited life expectancy or new onset systolic heart failure), or when an ICD must be removed (eg, infection).

Material and method: This article is the result of reviewing more than 6 articles related to the topic. A number of electronic databases including Clinical key, PubMed andChochranehost were queried and examined for potential sources using keyword searches. Predominantly, studies from within the past 2 years were considered most pertinent, though older articles were not discounted during the review.

Result: Data conflict on the utility of an implantable ICD early post-MI. A wearable cardioverter-defibrillator (WCD) has been suggested as temporary therapy for patients at high risk for SCD. The randomized VEST trial in patients with an acute MI and LVEF $\leq 35$ percent compared use of a WCD (begun within seven days of hospital discharge) plus usual medical treatment to standard medical treatment alone. Over an average follow-up of 84 days, the primary outcome of arrhythmic death was not different for the two groups. Compliance with medical therapy was excellent in both groups, likely contributing to fewer than expected events, while compliance with WCD usage was markedly lower than expected.

Conclusion: In cases where ICD implantation must be deferred, a WCD offers an alternative approach for the prevention of SCD.

\section{Impact of Door to ECG on patients refer to the emergency department with chest pain}

Zakerimoghadam M. ${ }^{1}$, Javadinejad M. ${ }^{1}$,
Ahmadi S. ${ }^{1}$, Tahmasvand M. ${ }^{1}$, Seylani K. ${ }^{1}$

${ }^{1}$ Tehran University Of Medical Sciencesnursing And Midwifery School, Critical Care Nursing, Tehran, Iran

\section{Abstract}

Backgrounds: Chest pain is the second most common reason for referral to an emergency unit. The American Heart Association recommends all patients with chest pain presenting to the emergency unitshould receive an ECG within 10 minutes of presentation. This is considered as the first line of diagnostic tests for myocardial infarction.

Material and method: . In this narrative review study, several databases including SID, Pub med, Google Scholar, Web of Science and Scopus ware used to collect data and search the articles published between 2000 and 2018. The keywords used to search articles were "door to ECG", "chest pain", "emergency unit" and "triage". The articles selected for review were type of intervention and descriptive in Persian or English, with available full text. The criterion for selecting articles was the similarity of the their subject with the search words including door to ECG (DTE) 
time and triage and their value in the early diagnosis and treatment of coronary disorders.

Result: A total of 20 articles were found. After removing the articles that didn't meet the inclusion criteria, 11 articles related to the subject of the study finally reminded in review. The significant outcomes of timely triage, appropriate time to perform ECG, barriers to access and suggestions for faster treatment by the system especially the role of nurses, were investigated.

Conclusion: The reduction of triage time and door to ECG (DTE) time as well as the role of the nurse in those cases are associated with a reduction in the complications and mortality of cardiac patients.

\section{Hospital Facilities at Home for Heart Failure Patients A cost-effectiveness study}

\author{
Khaleghparast S. ${ }^{1}$, Maleki A. ${ }^{2}$, Taghavi S. ${ }^{1}$, \\ Amin A. ${ }^{1}$, Maleki M. ${ }^{1}$, Oveisi M. ${ }^{1}$, Ghanbari B. ${ }^{3}$, \\ Hanifi Z. ${ }^{1}$, Naderi N. ${ }^{1}$
}

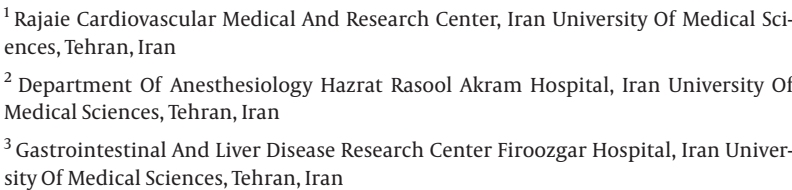

${ }^{2}$ Department Of Anesthesiology Hazrat Rasool Akram Hospital, Iran University Of Medical Sciences, Tehran, Iran

${ }^{3}$ Gastrointestinal And Liver Disease Research Center Firoozgar Hospital, Iran University Of Medical Sciences, Tehran, Iran

\section{Abstract \\ Backgrounds: Heart failure imposes an enormous economic burden on the healthcare sectors. The present research aims to study the establishment of a home care system for heart failure patients in order to evaluate the cost-effectiveness of this system and patient satisfaction. \\ Material and method: The present study is a health system research. After establishing it, 40 patients were selected as the sample with eligible criteria. The care was provided by nurses based on the physician's instructions. In the first visit at home, a questionnaire on the quality of life was filled out by patients or nurses. The financial data of medical records of patients was the reference for the analysis of cost. After following the inter- vention, the questionnaire on the quality of life was filled out by patients once again and their satisfaction was measured. The data were statistically analyzed using Python programming lan- guage and SPSS-16 at the 0.05 level of significance. \\ Result: The length of stay in the hospital for each patient re- duced from 2.1 days to 0.9 days per month. The number of an- nual hospitalization also reduced from 5 to 3 and the number of annual outpatient visits showed a reduction from 46 to 38 for each patient. The results of patient satisfaction assessment also indicated that most of patients were satisfied with services pro- vided to them. \\ Conclusion: The results showed that our study was cost effec- tive. It is suggested interventions are performed on larger scales. \\ Assessment The Role of Nurses in Promoting Lifestyle in Patients with Heart Failure A Review Study}

\author{
Pashaie S. ${ }^{1}$, Hosseini M. ${ }^{1}$, Pashaie S. ${ }^{2}$
}

${ }^{1}$ School Of Nursing And Midwifery, Shahid Beheshti University Of Medical Sciences, Tehran, Iran

${ }^{2}$ Nursing Faculty, Aja University Of Medical Sciences, Tehran, Iran

\section{Abstract}

Backgrounds: Major problems in the community include heart failure, which has been the cause of many of these patients' discomfort. Many factors in the lifestyle of these patients are involved. In this research, it has been attempted to address the role of nurses in improving the lifestyle of these patients.

Material and method: The present study is a comprehensive search or a combination of the keywords" nurse"," life style ", "patients", "Heart failure". Data collected from the PubMed and Scopus databases, ScieneDirect and Ovid sites between 2010 and 2018.

Result:The results of the study on the literature showed that the current survival rate of these patients has increased with the development of modern science in the treatment of patients with Heart failure. Many factors, such as Training on attitude and mood of the patients, Self-care education such as doctor's advice and regular daily activities and the consumption of healthy foods and medications regularly, control the symptoms of the disease and conduct regular monitoring of patients and timely referral of patients to health centers promotes the lifestyle of patients and hence improves the quality of life of these patients of these patients.

Conclusion: The results showed that nurses play an important role in improving the lifestyle of these patients. The main task of the nurse is to educate these patients. Therefore, it is suggested that programs should be developed to increase the knowledge of nurses about heart disease in order to have a positive impact on the life of patients with heart failure.

\section{Assessment of Factors Impacting the Quality of Life in Patients with Hypertension Systematic Review Literature}

\author{
Pashaie S. ${ }^{1}$, Hosseini M. ${ }^{1}$, Pashaie S. ${ }^{2}$ \\ ${ }^{1}$ School Of Nursing And Midwifery, Shahid Beheshti University Of Medical Sciences, \\ Tehran, Iran \\ ${ }^{2}$ Nursing Faculty, Aja University Of Medical Sciences, Tehran, Iran
}

\section{Abstract}

Backgrounds: Of the common diseases of the last century, blood pressure can be mentioned. It affects many people of different ages and reduces the quality of life of the affected patients. In this research, attempts have been made to investigate ways to improve the quality of life of these patients.

Material and method: The present study is a comprehensive search or a combination of the keywords "quality of life", "patients", "hypertension”, "Heart”. Data collected from the PubMed and Scopus databases, ScieneDirect and Ovid sites between 2010 and 2018. Result: The results of the study on the literature showed that the current survival rate of these patients has increased with the development of modern science in the treatment of patients with hypertension, but this increase in survival is often not associated with good quality of life in these patients, and sometimes with Many problems are encountered. Many factors, such as Empowerment of the patient and family, Stress management, increased spiritual 
wellbeing and life expectancy, Regular physical activity and healthy diet, Healthy lifestyle education have increase the quality of life of these patients. Also, everyday behaviours such as smoking and sleep disorder have led to a decrease in the quality of life.

Conclusion: The findings of this study revealed that there were significant factors affecting the quality of life of patients with hypertension, so that the seemingly simple and non-essential factors had a significant effect on the quality of life of these patients. The quality of life and survival of patients with hypertension are two dependent factors, which increases the quality of life of these patients, life expectancy will also increase. Therefore, it is recommended that a study be carried out in this country in order to investigate these factors in Iranian patients, as well as educating the medical staff and families of these patients and, most importantly, educating the patient himself to increase selfefficacy and having Agood life is a continuation of the disease.

\section{Guide line by JNC-8 in patients who referred to health centers survey of accumulation of hypertension treatment with last related to Fasa university of medical sciences 2017}

\section{Ordibehesht N. ${ }^{1}$ \\ ${ }^{1}$ School of Nursing And Midwifery Estahban Azad University, Department Of Nursing, Shiraz, Iran}

\begin{abstract}
Backgrounds: choice of antihypertensive drug treatment of hypertension is the most important .The study was done in patients who referred to health centers survey of accumulation of hypertension treatment with last related to Fasa university of medical sciences 2017.

Material and method: in this cross-sectional study population consisted of 200 patients with hypertension who were non random manner .Data collection toll was a questionnaire containing demographic and use of anti-hypertension medication on guide line JNC-8respectively.After review and approval of content validity and reliability of the questionnaire .Data and descriptive statistics were analyzed by SPSS-version 22 .

Result: 60 percent of patients with stage -1 , hypertension and 40 percent in stage-2,hypertention the most common medications in stage-1,hypertension, beta-blockers(atenolol) respectively .ACEI and thiazide on stein commonly prescribed drugs were used for stage-2, hypertension treatment, which was consistent with the guide line JNC-8.patients with diabetes and heart failure with underlying cases of beta-blocker drugs used to treat the guide-line JNC-8 not match.

Conclusion: treatment of hypertension patients with underlying causes JNC-8 guide line dose not match. It is a advised to go to their with training closed for new treatments. That improve patients outcome are more informed.
\end{abstract}

\section{Evaluation of marital stress based} on ejection fraction in patients with ischemic heart disease

\author{
Ghanbari Afra L. ${ }^{1}$, Ghanbari Afra M. ${ }^{2}$, \\ Shahbazpour J. ${ }^{3}$
}

${ }^{1}$ Kamkararabnia Hospital Qom University Of Medical Sciences, Qom University Of Medical Sciences, Qom, Iran

${ }^{2}$ School Of Nursing Midwifery, Iran University Of Medical Science, Qom, Iran

${ }^{3}$ School Of Nursing Midwifery, Qom University Of Medical Sciences, Qom, Iran

\section{Abstract}

Backgrounds: Now days marital relationships is an important topic in the study of health care system and social health system. and it can be influenced by several factors. The aim of this study is evaluation of the marital stress based on ejection fraction in patients with acute myocardial ischemia

Material and method: This cross-sectional study was performed on patients with ischemic heart disease admitted to angiography ward in one of hospital in Qom University of Medical Sciences in 1394. 300patients selected by convenience method and entered the study. Data were collected by the "demographic and clinical" and "Stockholm marital stress" questionnaires. The data were analyzed from central indexes and Univariate test by SPSS13 software

Result: The average age of participants was $55.24 \pm 6.06$ and $57.7 \%$ of them were male. Angiography results showed that $50 \%$ of participants were normal coronary, $16.3 \%$ of them were single vessel disease, $16 \%$ of them were tow vessel disease, and $17.7 \%$ of them were three vessel disease. $25 / 4 \%$ and 39/7\%, respectively, were not history of underlying diseases and drug user. The average of marital stress in patient with ejection fraction $>55 \%$ was $20.44 \pm 3.41$, in patients with ejection fraction $55-45 \%$ was $20.64 \pm 2.60$. In patients with ejection fraction $35-45 \%$ was $51 / 1 \pm 05 / 20$ and in patients with ejection fraction $<35 \%$ was $53 / 2 \pm 49 / 20$ ( $\mathrm{P}=0.001$ ). Conclusion: The results of this study indicated that patient with ejection fraction $<35 \%$ have less marital stress than other people. Therefore, it is recommended that health care providers, including nurses provide the ways for following-care and appropriate education for patients to with ejection fraction $<35 \%$.

\section{Catecholamines and broken heart syndrome Are catecholamines guilty With a look at nursing care}

\author{
Parchami F. ${ }^{1}$, Zakerimoghadam M. ${ }^{1}$, Seylani K. ${ }^{1}$ \\ ${ }^{1}$ Tehran University Of Medical Sciences, School Of Nursing And Midwifery, Tehran, Iran
}

\section{Abstract}

Backgrounds: The aims of this review article are investigating the role of catecholamines in the pathogenesis of Takotsubo cardiomyopathy (TCM) and related nursing care. TCM or broken heart syndrome is a acute acquired syndrome and characterized by acute reversible systolic dysfunction of apical and middle segments of the left ventricl in the absence of significant coronary artery occlusion. It is believed that emotional and physical stressors are the main drivers. Despite many efforts to better understanding this disorder, current knowledge is limited.

Material and method: Search for full text articles and case reports published between 2014-2018 were conducted with key words including "broken heart syndrome", "Apical ballooning syndrome", "Takotsubo cardiomyopathy", "Stress-induced cardiomyopathy", "catecholamines" and "nursing care", at PubMed, Google Scholar and Web of Science databases. Among 392 found articles and case reports, 21 articles were included in the study. Result: Among various hypotheses, catecholamine-induced microvascular dysfunction is the most accepted hypothesis. Stressful 
stimuli can increase the activity of hypothalamus - pituitary - adrenal axis, and triggers a pathophysiological response and acts as the basis for the development of TCM. So it is quite possible that prescribing exogenous catecholamines can provide these conditions. Conclusion: Although many studies have attributed some of the catecholamine toxicity to the pathophysiology, due to the potential impact on current treatment strategies and cardiovascular side effects, use of catecholamines requires more researches. Regarding to the similarity of the TCM symptoms with myocardial ischemia, accurate nursing care including early detection of symptoms, hemodynamic instability management, assessment of prescribed therapies, and electrocardiography are very important.

\section{Coronary artery bypass graft on- pump off pump and renal failure}

\section{Parizad R. ${ }^{1}$, Ghasemian Khojasteh Z. ${ }^{2}$, Khezerloy Agdam N. ${ }^{3}$, Taban Sadeghi M. ${ }^{3}$, Mousavi Shabestary M. ${ }^{2}$}

${ }^{1}$ Cardiovascular Research Center Tabriz University Of Medical Sciences Tabriz Iran ${ }^{2}$ Faculty Of Nursing And Midwifery Tabriz University Of Medical Sciences, Cardiac Nursing, Tabriz, Iran

${ }^{2}$ Cardiovascular Research Center Tabriz University Of Medical Sciences Tabriz Iran, Nursing, Tabriz, Iran

${ }^{3}$ Cardiovascular Research Center Tabriz University Of Medical Sciences Tabriz Iran, Cardiology, Tabriz, Iran

\section{Abstract failure in patients undergoing on-pump and off-pump. kidney function.The data were analyzed in SPSS version 21. and off-pump) in terms of renal failure incidence $(\mathrm{P}>0.05)$. neys in older patients may reduce this high risk complication. nine, off-pump surgery \\ Case Report Prosthetic valve thrombosis with INR5}

Backgrounds: Acute renal failure is a prevalent and serious disease that follows coronary artery bypass graft. One of the important symptoms of acute renal failure is the increased level of urea and serum creatinine. This study examined the rate of renal

Material and method: This paper is a descriptive-comparative study on 60 patients undergoing Heart. Levels of urea, creatinine, sodium, potassium and urinary output were controlled and recorded in the first days, admission and ICU discharge. Data collection tool made checklist: the first part included demographic information and the second part was related to information on

Result: Findings showed a statistically significant difference in terms of age and incidence of renal failure based on increased levels of urea and serum creatinine before and after coronary artery bypass graft $(\mathrm{P}<0.05)$. Findings also showed that renal failure significantly increased after coronary artery bypass graft based on levels of urea and serum creatinine $(\mathrm{P}<0.00)(\mathrm{P}<0.00)$. No significant difference was found between the two surgery procedures (on-pump

Conclusion: Patients' age was an important factor of renal failure following coronary artery bypass graft. Type of the surgery (on- and off-pump) and gender had no effect on the incidence rate of acute renal failure. Stronger measures to protect the kidKeywords: open heart surgery, acute renal failure, serum creati-

\author{
Ghorbani B. ${ }^{1}$, Saberi K. ${ }^{2}$, Bahramnezhad F. ${ }^{1}$, \\ Asgari P. ${ }^{1}$
}

${ }^{1}$ Tehran University Of Medical Sciences, Critical Care Nursing, Tehran, Iran

${ }^{2}$ Tehran University Of Medical Sciences, Medicine, Tehran, Iran

\section{Abstract}

Backgrounds: Objective: Considering that the mitral valve thrombosis is considered as a rare valve replacement problem, in a case of mitral valve thrombosis in the open heart care unit, the present study was conducted to express the desired case.

Material and method: Methods: This is a case study about a 50 year old woman who had replaced the mitral valve (mechanical) and replaced the trichospide valve 2018-9-29, Rasht , Iran. The patient was treated with warfarin and $\mathrm{ASA}(\mathrm{PT}=30 \mathrm{~s}$ and $\mathrm{INR}=5)$ which was referred to the hospital on 2018-10-28, due to dyspnea and shortness of breath. The patient was also subjected to fluoroscopy and, according to the report, one of theLeafletswas fixed. The patient was on an emergency list of surgery. The clot mass of 0.6 and $0.85 \mathrm{~cm}$ in the mitral valve ventral region was observed. MVPPG $=35$ and MVMPG $=28$ millimeters have been reported. The patient was surgically treated on 2018-1-11. During surgery, mitral valve repair and cleating of thrombosis were performed on valve.

Conclusion: In the first surgery, the correct selection of the heart valve according to the patient's condition will have a very important role in the prognosis of survival of patients, as well as the reduction of complications associated with artificial ventilation. The most common cause of biologic valve replacement, degeneration and mechanical valve is thrombosis.It is also important to the patient education about the proper use of anticoagulant drugs and dietary considerations.

\section{Association Body mass index waist circumference and waist hip ratio with cardiovascular risk}

\author{
Babapour S. ${ }^{1}$, Hoseini Marzango S. ${ }^{1}$ \\ ${ }^{1}$ Imam Khomaini Hospital, Master Of Critical Care Nursing, Freidonkenar Mazanda- \\ ran, Iran
}

\section{Abstract}

Backgrounds: Overweight and obesity have been recognized as a major risk factor for coronary artery disease (CAD). Although body mass index (BMI) has been a chosen method to measure body size in epidemiological studies, alternative measures, such as waist circumference (WC) and waist: hip ratio (WHR) which reflect central adiposity, might help to better assess body fat distribution and have been suggested to be superior to BMI in predicting CAD risk.

Material and method: The purpose of this study is to summarize the results of review studies and meta analyses on the relationship between abdominal obesity (waist circumference and waist hip ratio) and coronary artery disease (CAD). So a search strategy of keywords and Mesh terms used to identify review studies of cardiovascular risk and abdominal obesity from the PubMed, Google Scholar and Science Direct databases in from 2005 to 2018 in December 2018

Result: Overall 4 review studies and meta-analysis founded. The results of 2 analyses of Cao and et al (2018) and Coutinho and et al (2011) showed that a high WHR increased myocardial infarction (MI) risk and that elevated WHR is more strongly predictive of MI in women than in men. Also, central obesity was associated with mortality whereas, BMI was inversely associated with mor- 
tality. Central obesity was also associated with higher mortality in the subset of subjects with normal BMI and BMI $\leq 30 \mathrm{~kg} / \mathrm{m} 2$. The results of meta-regression analyses of De Koning and et al (2007) showed that for a $1 \mathrm{~cm}$ increase in WC, the relative risk of a CAD event increased by $2 \%$ after adjusting for age, cohort year, or treatment and for a $0.01 \mathrm{U}$ increase in WHR, the relative risk increased by $5 \%$. These results were consistent in men and women. Overall WHR was more strongly associated with CAD than that for WC, although this difference was not significant. Whereas, a review of the published evidence by Huxley and et al (2010) indicated that there is no clear agreement as to whether measures of central obesity are more strongly associated with cardiovascular morbidity and mortality compared with BMI.

Conclusion: There is a clear need for further long-term, large cohort studies to examine this issue further and this is better to incorporate measures of central obesity into CAD risk assessments for young to middle-aged adults and normal BMI. Further work is needed to figure out the best practice guidelines for obtain the various sizes of central obesity that contribute to CAD risk.

\section{Reasons for the termination of pregnancy in hospital women with heartdisease in Shahid Madani Heart center in Tabriz}

\author{
Pishnamaz Ahari N. ${ }^{1}$, Imani S. ${ }^{1}$, Yeganeh S. ${ }^{1}$, \\ Afshin Nia N. ${ }^{1}$, Zareh F. ${ }^{1}$ \\ ${ }^{1}$ Tabriz Medical Sciense University, Shahid Madani Heart Center, Tabriz, Iran
}

\begin{abstract}
Backgrounds: With regard to controls on the causes of maternal mortality in pregnant women, it has been noted in past studies that heart disease has become one of the most common causes of death in pregnant women in many countries, including Ira. To be therefore, it is necessary to closely monitor and control women with heart disease or women to have cardiovascular complications during pregnancy. The present study examines the causes of pregnancy termination in pregnant women admitted in the first half of 2018 in Tabriz.

Material and method: All women admitted in cardiology ward for pregnant women at the heart hospital witch were referenced from the entire province with a definite diagnosis of heart disease in a case series study were investigated.

Result: Out of 10 pregnancy terminations associated with pregnant women, the reasons for termination of pregnancy were HF, PTE, VF ICD, LV Dysfunction, MS, DCM, MR, with 4 cases leading to abortion and the rest of the cesarean section respectively. Shortness of breath and respiratory distress and palpitations of the heart and well- untreated pneumonia where the main cause of admission. No maternal death was reported during the study period and three months later.

Conclusion: Increasing the incidence of hospitalization for pregnant mothers due to heart disease. This indicates increased care and special attention in these cases. The strength of this care is reducing maternal death at the center.
\end{abstract}

\section{Educational needs from the} perspective of heart surgery patients

\section{Parizad R. ${ }^{1}$, Mousavi Shabestari M. ${ }^{1}$}

${ }^{1}$ Student Research Committee And Faculty Of Nursing And Midwifery Tabriz University Of Medical Sciences Tabriz Iran, Nursing, Tabriz, Iran

\section{Abstract}

Backgrounds: Cardiac surgery associates with potential physiological, mental, emotional, growth and spiritual consequences due to the existence of many stressful factors. Training and learning patients can decreas risk-creating and stressful factors. Such a learning approach should be designed based on patients' needs and such needs should be identified by nurses before to any learning. However, there are rare studies focusing on the identification of patients' learning needs. Thus, this study aims to determine patients' awareness of learning needs for Coronary Artery Bypass Graft (CABG).

Material and method: This is a descriptive study carried out within 6 months on 101 cases undergone CABG (36 females and 65 males) in Tabriz Shahid Madani education and treatment center. They were investigated at the time of discharging from ICU and surgery departments. Patients' needs were determined using Cardiac Patients' Needs Learning Inventory (CPNLI) tool.

Result: According to findings, patients give the most important priority to the drug index. The next learning needs perceived by patients are :"How will my heart be treated?" related to "anatomical and physiological learning needs category", "what kind of activities can be done to reduce heart attack chance?" related to "risk factors learning needs category" and "how patients' activity level can be increased?" related to "physical activity learning needs category".

Conclusion: The prerequisite for implementing any learning program is the investigation and measurement of learning needs. Therefore, before initiating any learning program nurses need to continuously assay learning needs of patients while considering patients' abilities.

Coronary Artery Bypass Graft, Educational Needs

\section{Congenital heart disease abnormalities in East west of Azerbaijan in 2018 A new approach to improve the pregnant mother with heart problems}

\author{
Imani S. ${ }^{1}$, Ziya Sarabi P. ${ }^{2}$, Pishnamaz Ahari N. ${ }^{1}$, \\ Pur Shahbazi Liqvan E. ${ }^{1}$, Mojtahedi M. ${ }^{3}$ \\ ${ }^{1}$ Tabriz Medical Sciense University, Shahid Madani Heart Center, Tabriz, Iran \\ ${ }^{2}$ Tabriz Medical Sciense University, Ms Biothecnology, Tabriz, Iran \\ ${ }^{3}$ Tabriz Medical Sciense University, Azar Shahr Health Depatment, Azar Shahr, Iran
}

\section{Abstract}

Backgrounds: Congenital malformations of heart since birth cased one of the factors influencing the mortality rate of children under one year, so this study is to evaluate new born with congenital heart disease in 1396 in the hospital in Tabriz, to identify and evaluated the types of cardiovascular abnormalities and the exact statistics of the disease well as the identification of risk factors for medical interventions

Material and method: After obtaining consent from at total of 326 cases examined in medical records of newborns born with congenital anomalies, 54 cases were diagnosed with congenital anomalies in Al-Zahra Tabriz hospital in 2018. Information such as age and gender of the baby and mother's heart abnormality 
and the location and method of pregnancy termination of pregnancy week spss software and analyzed them.

Result: In this study, 54 cases of birth defects with congenital heart abnormalities were identified with the highest number of heart problems (PDA+ASD) with $14.8 \%$ and ASD with $11.1 \%$ in the second place and ASD+VSD+PDA, each of them had the highest rates with $3.9 \%$ in the third place. Also TOF was the second most common heat disease with 5.6\%. The most cases of this type of abnormalities were observed at the age of 24and 31 years old, respectively. The statistic of girls were 28 cases and boys were 26 , out of which 35 were cleared and 9 cases of neonatal death and 9 were transmitted to the children's hospital for further interventions. In most cases, termination of pregnancy was carried out with cesarean section, and most of them were done at 36 weeks and also over 30 weeks.

In the case of just one termination 25 weeks and the weight at birth was 34 , the total number was 2500 grams and above. The highest APGAR of the first minute was 9 and the lowest APGAR was 2.

Conclusion: According to the results of this study, the necessary of identifying cardiac abnormalities based on the most cases of conflict and planing for timely medical interventions and also eliminating risk factors during pregnancy seems prerequisite to mothers.

\section{Correlation between arterial and venous blood gases in patients with mechanical ventilation after intracranial surgery}

Hoseini Marzango S. ${ }^{1}$, Barzabadi Farahani Z. ${ }^{2}$, Dabirian A. ${ }^{2}$, Pourhoseingholi M. ${ }^{3}$, Babapour S. ${ }^{4}$

\footnotetext{
${ }^{1}$ Imam Khomaini Hospital, Nursing, Freidonkenar Mazandaran, Iran

${ }^{2}$ School of Nursing Midwifery Shahid Beheshti University Of Medical Sciences, Nursing, Tehran, Iran

${ }^{3}$ Research Institute For Gastroenterology And Liver Diseases Shahid Beheshti University Of Medical Sciences, Associate Professor Of Biostatistics, Tehran, Iran

${ }^{4}$ Imam Khomaini Hospital, Master Of Critical Care Nursing, Freidonkenar Mazandaran, Iran
}

\section{$\underline{\text { Abstract }}$}

Backgrounds: Arterial blood sampling collects information about the amount of blood oxygenation, arterial blood carbon dioxide pressure and insurance of proper regulation of ventilator, is a gold standard and critical test in patients undergoing neurosurgery. As regards arterial puncture because of sampling is led to multiple complication for example: pain that increases intracranial pressure, bleeding, thrombosis, infection and disturbance of organ blood stream. Occasionally it would need great skill and also sometimes it is tried several times for sampling. Therefore sampling of peripheral vein can be a viable alternative for arterial sampling.This study aimed to evaluate the correlation between arterial and peripheral vein blood gases parameters consist of $\mathrm{PH}$, pressure of oxygen and carbon dioxide, hemoglobin saturation, bicarbonate concentration and the amount of BE / BD in patients after craniotomy that temporarily are admitted in intensive care unit and connected to ventilator.

Material and method: This study is a descriptive correlational study that conducted in 2014. 100 patients that after neurosurgery were admitted in ICU and temporarily connected to ventilator, selected as samples availability and was gotten arterial and peripheral vein sample simultaneously. The amounts of $\mathrm{PH}$, oxygen and carbon dioxide pressure, hemoglobin saturation, bicarbonate concentration and the amount of BE / BD Both were calculated and compared using a paired t-test. Then Pearson's correlation coefficient was calculated. Using the regression model, the arterial values were predicted on peripheral vein values and compared with the observed arterial values.

Result: as a result Comparison of arterial and peripheral venous values, Pearson correlation coefficient $(\mathrm{R})$ and the level of significance $(\mathrm{P})$ was calculated as follows: $\mathrm{PH}(0.91,0.001>), \mathrm{PO} 2(0.68$, $0.001>$ ), PCO2 (0.90, 0.001>), O2 Saturation (0.26, 0.009), $\mathrm{HCO} 3$ $(0.91,0.001>)$ and $\mathrm{BE}(0.94,0.001>)$.

Conclusion: Peripheral venous blood gas analysis of $\mathrm{PH}$, pressure of carbon dioxide, bicarbonate concentration and the amount of BE / BD can be a good alternative arterial blood gas test in neurosurgery patients are hemodynamically stable. But according to the low correlation and long confidence interval about hemoglobin saturation and oxygen pressure can't perform this replacement.

\section{The role of Vitamin C in the Prevention of Post operative Complication after Cardiac Surgery}

\author{
Shirvani Y. ${ }^{1}$, Yadegari M. ${ }^{1}$, Hamadani K. ${ }^{1}$, \\ Biat R. ${ }^{1}$, Mohamadi F. ${ }^{1}$, Karami A. ${ }^{1}$ \\ ${ }^{1}$ Zanjan Univercity Of Medical Since, Ayatolah Mosavi Hospital, Zanjan, Iran
}

\begin{abstract}
Backgrounds: In cardiac surgery with $\mathrm{CPB}$, vitamin C levels decrease with the production of reactive oxygen species (ROS) and remain low for days after surgery, indicating a greater demand of vitamin $C$ in the setting of surgery and $\mathrm{I} / \mathrm{R}$-induced oxidative stress. The purpose of this study was to evaluate the efficacy of vit $\mathrm{C}$ as prophylaxis for the prevention of postoperative Complication in cardiac surgery
\end{abstract}

Material and method: A systematic search of Google Scholar, PubMed, EMBASE, the Cochrane Library, and clinical trial registries, was performed.

Result: In the Cardiac surgery patients, who received vitC as prophylaxis, had a significantly lower incidence of postoperative Complication. In a systematic review and in 6 different meta-analyses including 8-15 RCTs, vitamin C was shown to significantly reduce the occurrence of postoperative cardiac arrhythmia, mainly atrial fibrillation $(\mathrm{P}<10-4)$.

Vitamin c treatment improves ventricular function, reduce vasopressor and fluid demand and increases the cardiac index. Oxidative stress and myocardial damage after cardiac surgery with CPB might be decreased by the administration of vitamin C. Conclusion: Our findings suggest that $\mathrm{Vit} C$ is effective as prophylaxis for the prevention of postoperative Complication. The administration of vit $\mathrm{C}$ may be considered in all patients undergoing cardiac surgery.

\section{Effect of Cardiopulmonary Bypass Machine on Serum Vitamin D3 Level in Pediatrics Open Heart Surgery review}

\author{
Shefaat S. ${ }^{1}$, Alikhani Boroujeni R. ${ }^{2}$
}


${ }^{1}$ Isfahan University Of Medical Sciences, Faculty Of Medicine, Isfahan, Iran

${ }^{2}$ Isfahan University Of Medical Sciences, Nursing, Isfahan, Iran

\section{Abstract \\ Backgrounds: Definitive treatment for most children with congenital anomalies of the heart surgery. The type and extent of the surgery will vary according to the type of defect and con- dition of the child. Cardiac surgeries in congenital ailments are largely open and In open heart surgery, there is a need to stop the functioning of the heart in order to repair the lesions inside and outside of the heart, and during this surgery, the cardiopul- monary bypass machine is used to create an external blood flow. The purpose of this article is to provide a general overview on observational studies and clinical trials on the effects of cardio- pulmonary bypass machine on serum levels of vitamin $\mathrm{D}_{3}$ in pediatrics open heart surgery. \\ Material and method: The results of this study are the search for articles from the beginning of 2010 to December 2017 at the Pubmed, Scopus and Science Direct databases. Key words used, vitamin D3, cardiopulmonary bypass machine, pediatrics open heart surgery \\ Result: Different articles showed a significant relationship be- tween electrolyte disturbances and the patient's use of the car- diopulmonary bypass machine. A decrease in vitamin D3 levels has been seen with increased use of the vasopressor during use of the heart and lung pump and later in patients. \\ Conclusion: Considering the importance of having sufficient amounts of vitamin $\mathrm{D}_{3}$ in the body and the effect of its deficiency on the cardiovascular system, the probability of a relationship between the mortality rate after open heart surgery with vita- min D3 level is raised. \\ - Professionalism is a factor in maintaining a perfusionist society and promoting it}

\author{
Salmani S. ${ }^{1}$, Hemati M. ${ }^{1}$ \\ ${ }^{1}$ Valiasr Subspecialty Police Hospital, Heart Surgery, Tehran, Iran
}

\begin{abstract}
Backgrounds: Commitment to principles was the simplest definition of being professional Professionalism is marked with professional knowledge or skills. This marking gives special privileges to the occupier and affects his individual identity and creates a new identity for him. As it applies to all of his individual facets, in order to finally identify each other alongside each other a set of professional people and defines a professional society. On the other hand, the society expects them to see the benefits of society to their own interests. Medical, judging and teaching from the distant past are a set of professional people who prefer the interests of others and they commit their sacred purpose in the form of an oath.

Material and method: The opposite of professionalism is amateurism. An amateur for spending time without social commitment optimizes his leisure time while he does not have the full skill in his field of work and only uses existing facilities, but the professional on the frontier of knowledge and progress He considers himself to be required to progress and to achieve pure
\end{abstract}

knowledge. He considers these actions as his duty and does not seek time

Professional begins with accountability.The perfusionist sees himself as part of a larger system that offers sophisticated and vital care, which must always be functional at peak performance. Volosky states that professional behavior is one of the most difficult areas in medicine for evaluation.

Although there are many approaches to evaluating professional behavior, there are still no valid and reliable measurements to measure professional behavior.

Result: However, we can specify the minimum. Among them, ideal behavior, desirable, expected, undesirable behavior, abusive behaviors as criteria, as well as commitment to professional competence, honesty in work, patient privacy, maintaining a good relationship with patients, improving the quality of services and care, scientific knowledge Maintaining trust through managing conflict of interests and professional responsibilities, and ultimately defining a general framework for professionalism among perfusionists.

Conclusion: In order to carry out professionalism, it is necessary to use an appropriate evaluation tool for various reasons of peer evaluation in the Iranian perfusionists community of the most appropriate methods.

Without specifying the goal, the tool is an imperfect and low-impact tool, improving quality as the goal in delivering the most comprehensive goal. Therefore, it is suggested that in the Iranian perfusionists' society with the aim of improving the quality of specialized services through peer to peer evaluation and the specific framework of ethical codes of professionalism, it is expressed and expressed because this concept is in the depths of the professional personality of this society and is there but It is not shown as deserving. This article describes the topics mentioned.

\section{- Chest pain in children}

\section{Rastgar Koutenaei F. ${ }^{1}$, Jahantigh Akbari N. ${ }^{2}$, Ali Rezaei O. ${ }^{3}$}

${ }^{1}$ Phd Student Of Iran University Of Medical Sciences, Physiotherapy, Tehran, Iran

${ }^{2}$ Phd Student Of Shahid Beheshti University, Physiotherapy, Tehran, Iran

${ }^{3}$ Tehran University Of Medical Scienses, Physiotherapy, Tehran, Iran

\section{Abstract}

Backgrounds: Chest pain is a major problems among children. The aim of this study was to identify and study causes of chest pain in children and correct diagnosis can be chosen for the treatment of children.

Material and method: A literature search for the period of 1996-2018 was performed, using PubMed ,Science Direct ,Google Scholar databases.

Result: 15 articles were selected according to the inclusion criteria of the study. four studies were about chest pain in focal musculoskeletal disorders and six studies were about psychiatric condition and three studies were about gastroesophageal reflex and tow studies were about cardiovascular disorders.

Conclusion: The incidence among children is unknown, Chest pain affects children of all ages, with a peak incidence between 12 and 14 years. The causes of chest pain in children are classified as idiopathic, musculoskeletal, pulmonary, cardiovascular, gastrointestinal, psychogenic and malignant. Cardiac disorders are uncommon causes of chest pain in children but are potentially 
the most serious causes in children. Musculoskeletal pain arising from the chest wall accounts for $20 \%$ to $30 \%$ of chest pain in children and is the most common identifiable origin. A variety of musculoskeletal syndromes have been described as causes of chest pain in children, including pectoral syndrome, coracoid process syndrome, trapezius syndrome, latissimus dorsi syndrome, and serratus anterior syndrome, Tietze's syndrome and xiphoid syndrome. Most causes can be diagnosed from history and physical examination.

Keywords: Chest pain, pediatric, children, musculoskeletal

\section{The first result of the Iranian Registry of Atrial Fibrillation (IRAF)}

\author{
Heidarali M. MD ${ }^{1}$, Haghjoo M. MD ${ }^{2 *}$, Bakh- \\ shandeh H. MD ${ }^{1}$, Farjam Fazelifar A. MD ${ }^{2}$, \\ Alizadeh A. MD ${ }^{2}$, Emkanjoo Z. MD ${ }^{2}$, Madadi Sh. \\ $\mathrm{MD}^{2}$, Golpira R. MD ${ }^{2}$
}

${ }^{1}$ Rajaie Cardiovascular Medical and Research Center, Iran University of Medical Sciences, Tehran, Iran

${ }^{2}$ Cardiac Electrophysiology Research Center, Rajaie Cardiovascular Medical and Research Center, Iran University of Medical Sciences, Tehran , Iran

\begin{abstract}
Background: Atrial fibrillation (AF) is the most common sustained arrhythmia and its prevalence is likely to increase prominently in the next decades. AF is a common cause of stroke, heart failure, hospitalizations and death in affected patients. The management of AF has been markedly changed in the recent decade. There is not enough data on patient characteristics, practice patterns and outcomes of AF in IRAN. The study objective was to investigate the clinical presentation, management and outcome in patients with AF who were prospectively enrolled in Iranian Registry of Atrial Fibrillation(IRAF) General Registry.

Materials and methods: IRAF is a prospective observational cohort of patients with AF within a 12-month follow-up. A total of 738 consecutive patients were enrolled between February 2018 to February 2019 in Rajaie Cardiovascular Medical and Research Center. Data was collected on a standardized case report form and entered offline and online. Data collected included patient demographics, medical history, AF type, echocardiography and ECG, treatment, and outcomes. As patients were admitted, details of their treatment, investigations, and outcomes during hospital stay were collected. Our report is the cross-sectional phase of cohort study on 738 cases. Inform content was taken from all the patients.

Results: Totally 738 patients [ $60 \%$ male; mean age: $58.44 \pm 14.37$ were enrolled. All demographic and clinical data characteristics were assessed. Palpitation was the most common complaint among AF cases (69\%). $41.1 \%$ of AF cases were in NYHA FC II. The most frequent risk factors were hypertension $51.6 \%$, hyperlipidemia $36.3 \%$ and Diabetes mellitus $27.5 \%$. The most common concomitant disease was Valvular heart disease (65.2\%). 13.8\% of AF cases were asymptomatic. The median (IQR) of LVEF, LA diameter and international normalized ratio (INR) were 50\% (45\%-55\%), $4 \mathrm{~mm}$ (3.4-4.4), and 1.2 (1.03-1.7) respectively. The most of the $\mathrm{AF}$ cases underwent replacement of ICD (13.3\%). 34.4. \% of AF cases had the history of PCI and 36.6\% underwent CABG. $45.1 \%$ had CAD. The great number of AF cases had two vessels involvements (57.2\%). The most common type of AF was paroxysmal in $61.7 \%$ of the cases. $71.8 \%$ of the AF patient had CHADS-VASC score $\geq 1$ and
\end{abstract}

majority of the patients were at low risk for bleeding related to anticoagulant therapy (HSB-BLED score $<3$ in $99.9 \%$ ).

The most widely used anticoagulants were ASA (56.6\%), Warfarin (55\%) and Rivaroxaban (55\%). Antiarrhythmic agents were used in $77.5 \%$ of the AF cases. Out of those, Beta-blocker was used in most of the AF cases (80.2\%). Amiodarone (45.9\%), Felecainide (44.3\%) and Sotalol (39.7\%) were the most prevalent antiarrhythmic drugs in AF cases. Antihypertensive was used in $47.6 \%$ of the AF cases. $46.7 \%$ of the AF cases had history of at least one electrical cardioversion. Chemical cardioversion performed in $34.9 \%$ of the AF cases. Catheter ablation was performed in $86.1 \%$ of the AF cases, including RF PV isolation (75.7\%), Cryo PV isolation (24\%) and AV node ablation (7.9\%). Regarding thromboembolic events7.3\% of the AF cases had transient ischemic attack or cerebrovascular accident and $1 \%$ had other organ thromboembolic events. Regrading non-Major bleeding, 10.9\% had epistaxis, $9 \%$ had skin bruise or hematoma, and $0.1 \%$ had limb bleeding. None of the cases had hematuria. With regards to major bleeding, $0.3 \%$ of the AF case had gastrointestinal bleeding. 8.1\% of the cases had intracranial bleeding and $0.1 \%$ had intraocular bleeding. $6.6 \%$ of the AF cases had MI. 5.3\% had tachy-induced cardiomyopathy. Conclusion: IRAF first results provide the complete clinical status of the AF cases which could provide the useful information that may eventually lead to health policy decision-making. Keywords: Atrial Fibrillation; Anticoagulants, Antiarryhthmia Agent; CHADS (Congestive heart failure, Hypertension, Age; Diabetes and stroke/TIA-VASC (vascular disease); Cryosurgery; Electrical Cardioversion, HAS-BLED Hypertension, Abnormal liver renal function, Stroke history, Bleeding history or predisposition, Labile INR, Elderly, Drug/alcohol usage, Radiofrequency Ablation, Registry

Trend of salt intake measured by 24hour urine collection samples among Iranian adults, 1998-2013: The Isfahan Salt Study

\author{
Mohammadifard N.*, Khosravi A., Nouri F., \\ Jozan M., Sarrafzadegan N.
}

Isfahan Cardiovascular Research Center, Cardiovascular Research Institute, Isfahan Univesity of Medical Sciences, Isfahan, Iran

\section{Abstract}

Background and aim: Few population-based studies conducted in the Eastern Mediterranean region assessed salt intake by the measurement of 24-hour (24-h) sodium urine excretion. The current study aimed to assess the trend of salt intake in Iranian adults between 1998 and 2013, and examined the relationship between urine excretion of sodium (Na) and potassium (K) with blood pressure (BP).

Methods: These cross-sectional studies were performed on 564 , 157,509 and 837 randomly selected healthy adults aged $>18$ years from Isfahan city, Iran, in 1998, 2001, 2007 and 2013, respectively. BP was measured using a mercury sphygmomanometer according to a standard protocol. Single 24-h urine was collected to assess 24-h urinary $\mathrm{Na}$ (24-hUNa) as a surrogate of salt intake, and 24-h urinary K (24-hUK).

Results: The estimated trend of salt intake was 9.5, 9.7, 9.6 and $10.2 \mathrm{~g} /$ day in total population $(\mathrm{P}<0.001)$. The increase in salt intake between 1998 and 2013 was significant only in in men, (P $<0.001)$. 
Conclusions: This population-based study indicated that salt intake was about two times of recommendation in Isfahan city, Iran, and suggest that it would be essential to implement a salt reduction strategy program in Iranian population. Longitudinal national studies with larger samples examining the trend of salt intake are warranted.

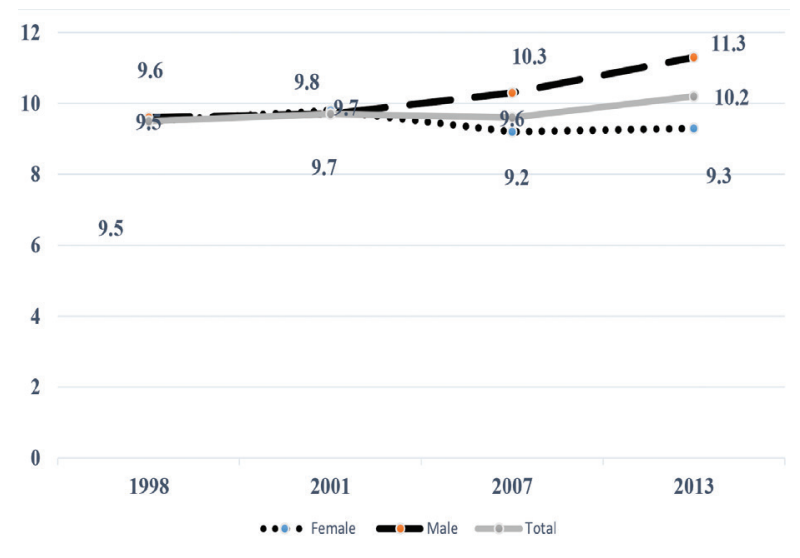

Trend of salt intake among adult population of Isfahan city, Iran 1998-2013

\section{Multi-Center, Multi-disciplinary, Cardio-Oncology Toxicity Registry (COT-R)}

\author{
Alizadehasl A. ${ }^{1}$, Salmanian S. ${ }^{2}$, Maleki M. ${ }^{3}$, Noohi F. \\ Anbiaie R. ${ }^{5}$, Golpira R. ${ }^{6}$, Mofid M. ${ }^{7}$, Berahman M. ${ }^{8}$, \\ Azadeh P. ${ }^{9}$, Foudazi H. ${ }^{10}$, Mohebbi B. ${ }^{11}$, \\ Malekzadeh M. ${ }^{12}$, Ghadrdoost B. ${ }^{13}$, Mokhtaran M. ${ }^{14}$, \\ Oveisi M. ${ }^{15}$, Nabhani F. ${ }^{16}$
}

${ }^{1}$ MD, FACC, FASE; Professor of Cardiology, Iran University of Medical Science, Rajaie Cardiovascular,Medical and Research Center

${ }^{2} \mathrm{MD}$; Assistant Professor of radio-oncology, Iran University of Medical Science, Haft Tir General Hospital

${ }^{3}$ MD, FACC; Professor of Cardiology, Iran University of Medical Science, Rajaie Cardiovascular, Medical and Research Center

${ }^{4}$ MD, FACC, FASE; Professor of Cardiology, Iran University of Medical Science, Rajaie Cardiovascular,Medical and Research Center

${ }^{5} \mathrm{MD}$; Assistant Professor of radio-oncology, Iran University of Medical Science, Haft Tir General Hospital

${ }^{6} \mathrm{MD}$, Rajaie Cardiovascular,Medical and Research Center

${ }^{7} \mathrm{MD}$, Associate Professor of radio-oncology, Shahid beheshti University of Medical Science, Labbafi nejad General Hospital

${ }^{8} \mathrm{MD}$; Assistant Professor of radio-oncology, Iran University of Medical Science, Firoozgar General Hospital

${ }^{9} \mathrm{MD}$, Associate Professor of radio-oncology, Shahid beheshti University of Medical Science, Emam Hossein General Hospital

${ }^{10} \mathrm{MD}$, Associate Professor of radio-oncology, Shahid beheshti University of Medical Science

${ }^{11} \mathrm{MD}$; Associate Professor of Cardiology, Rajaie Cardiovascular, Medical and Research Center

${ }^{12} \mathrm{MD}$, Assistant Professor of radio-oncology, Shahid beheshti University of Medical Science, Shohadaie Tajrish General Hospital

${ }^{13}$ PHD of Phisioiology, Iran University of Medical Science, Rajaie

Cardiovascular,Medical and Research Center
${ }^{14} \mathrm{MD}$, Rajaie Cardiovascular,Medical and Research Center

${ }^{15} \mathrm{MD}$, Especialist in Artificial intelligence; Rajaie Cardiovascular, Medical and Research Cente

${ }^{16}$ Bachelor in Nursing, Rajaie Cardiovascular,Medical and Research Center

\section{Abstract}

Introduction: Chemo-therapy and radiation therapy-induced cardio-toxicities are a major cause of morbidity and mortality in people living with or surviving cancer. All antineoplastic agents and radiation therapy target tumor cells but also result in collateral damage to other tissues including the cardiovascular system. The Multi-Center Multi-disciplinary Cardio-Oncology Toxicity registry (COT-R) was formed to address the demographical, clinical and para-clinical aspects of all types of Cancers, their various and unavoidable treatments in relation with CARDIOVASCULAR involvement in this process with the aim of reducing the cardio-toxicity and improving the diagnosis and timely management of it. Methods: Eight centers are included in the our COT registry. This database has a web-based interface by ERAF, a secure web application for building and managing online databases. It is specifically geared to support data capture and also data analysis for research studies. Data elements currently being collected from the 8 participating centers will be reviewed every 6 months.

All data obtained from participating centers will be used in the development of mathematical predictive models of cardio-toxicity by machine learning and artificial intelligence.

Also several clinical researches will be designed based on registry data. In near 2 years till now about 400 patients were enrolled and we analyzed the preliminary data in 200 patients especially in advanced echocardiography field for presenting in this congress.

Results: Totally, 200 patients at a mean age of $41 \pm 22$ years were enrolled. Female gender, age $>60$ years old, history of Hypertension, Diabetes Mellitus , $>500 \mathrm{mg} / \mathrm{m} 2$ usage of Adriamycin, rapid infusion of Adriamycin, and so importantly moderate or more than moderate left heart valvular heart disease and left ventricular hypertrophy are the main risk factors for cardio-toxicity in our study.

And as sample report: Between the left ventricular (LV) functional parameters, $\mathrm{LV}$ circumferential strain is a stronger parameter for detection of subclinical involvement. (of course we didn't assess the LV3D EF and also LVEF by heart model software yet).

We found right ventricular (RV) dysfunction in $78 \%$ of patients with LV dysfunction ( $>15 \%$ reduction in RV global strain), and In $15 \%$ of patients RV dysfunction develop before the LV dysfunction.

In $12 \%$ of our patients mitral valve was involved with at least one grade increase in Mitral regurgitation (MR). Specially, rate of increase is significant in patients with MR basic.

And importantly, we had very good result in preventing and managing the cardio-toxicity by Carvedilol, Lisinopril and Spironolactone.

Conclusion: The effect of cardio-toxicity on morbidity, mortality, and community resources is very high. Our first and last goal of this registry is improvement of prevention, diagnosis and management of cardio-toxicity.

Our results demonstrated that Baseline and follow-up cardiovascular assessment, preventive strategies and good and timely management as a result of close Cooperation between cardiologists and oncologists is absolutely essential in Significant reduction of cardiovascular morbidity and mortality in cancer patients.

Keywords: Cardio-toxicity, Registry, Cancer, Cardiovascular, Cardiac mortality, Cardiac morbidity 


\section{- Comparison of 2 days oral Acetaminophen and oral Ibuprofen on the closure of patent ductus arteriosus PDA in premature neonates}

\author{
Ghaderian M. ${ }^{1}$, Barekatain B. ${ }^{2}$, Banazade A. ${ }^{1}$ \\ Mehdi Ghaderian - Pediatric Cardiovascular Research Center Cardiovascular Research \\ Institute Isfahan University of Medical Sciences Iran, Isfahan \\ ${ }_{2}^{2}$ Pediatric department Emam Hosein Hospital Isfahan University of Medical Sciences \\ Isfahan Iran Iran, Isfahan
}

\section{Abstract}

Backgrounds: The purpose of this study was to compare the effectiveness of Ibuprofen and Acetaminophen in the closure of patent ductus arteriosus (PDA), which is commonly occurred in fifty percent of the neonates with less than 1000 grams.

Material and method: Forty preterm neonates (their age range was less than 23 weeks), who were admitted in Shahid Behesti and Al-Zahra hospitals (Isfahan, Iran), were included in this study. The weight of the mentioned patients was more than 1000 grams, and they were suffer from symptomatic PDA. Fifty percent of the preterm neonates received $10 \mathrm{mg} / \mathrm{Kg} / 6 \mathrm{~h}$ of Acetaminophen. The other patients, furthermore, received 10-5-5 mg/ Kg of Ibuprofen in 3 days, and the results (the vital sign and echocardiography findings) were compared to each other.

Result: In the Acetaminophen group sixteen patients $(80 \%$, p $<0.001)$ responded to the drug. Furthermore, seventeen $(85 \%$, $\mathrm{p}<0.001$ ) neonates patients who were included the Ibuprofen group, responded to this drug. Also, three patients of the Acetaminophen group responded to the other studied drug (Ibuprofen) in the next stage, and two neonates of the Ibuprofen group responded to other studied drug (acetaminophen).

Conclusion: According to results of this study, $10 \mathrm{mg} / \mathrm{Kg} / 6 \mathrm{~h}$ of Acetaminophen for two days have similar effects in the closure of PDA in comparison with previously suggested three days intravenous and is equally effective in the closure of PDA in the mentioned patients.

\section{Evaluation the effect of premedication with intravenous paracetamol on hemodynamic changes during laryngoscopy in children with heart diseases}

\author{
Ahmadipour M. ${ }^{1}$, Ahmadinejad M. ${ }^{1}$ \\ 'kerman university of medical sciences Iran, kerman
}

\section{Abstract}

Backgrounds: Tracheal intubation can be associated with considerable pediatric hemodynamic changes, particularly in patients with heart diseases due to its sever pain and stimuli of oropharynx

.The aim of this double blind clinical trial study was to assessment hemodynamic alterations following tracheal intubation by premedication with paracetamol which is a safe analgesic drug in children.

Material and method: After obtaining approval from the Eth- ics Committee of Kerman University of Medical Sciences, and obtaining informed consents from the patients Legal guardians , a total 60 pediatric patients (3-10 years old age) with cardiac disease who was undergoing for noncardiac elective surgery were randomly allocated to either the parastamol or the placebo premedication groups.

The patients were intubated by a single experienced anesthesiologist. After oxygenation with 100\% oxygen for three minutes, patients were administered midazolam $0.05 \mathrm{mg} / \mathrm{kg}$ and intravenous fentanil $2 \mu \mathrm{g} / \mathrm{kg}$. Induction of anesthesia was made with propofol $1.5 \mathrm{mg} / \mathrm{kg}$ and cisatracurium $0.15 \mathrm{mg} / \mathrm{kg}$. in the patients of case group $15 \mathrm{mg} / \mathrm{kg}$ of parastomol was administered intravenously, and in control group placebo was administered.

The mean arterial pressure (MAP) pulse rates (PR), rate-pressure product (RPP) data, were collected at; baseline, 1 and 5 minutes after intubation.

Result: Mean arterial pressure (MAP) decreased similarly after induction in both groups; (16.75 \pm 19.73 in the paracetamol group and $14.03 \pm 18.98$ in the placebo group; P-value $=0.72$ ),RPP reduction was similar in both groups; $(2.27 \pm 6.73$ in the paracetamol group and $2.14 \pm 4.73$ in the placebo group; P-value $=0.93$ ) whereas heart rate (HR) did not significantly decreased in both groups $(12.33 \pm 23.73$ in paracetamol and $14.65 \pm 24.73$ in placebo group; P-value $=0.37$ ) One minute after intubation HR significantly increased in placebo group more than paracetamol group $(28.95 \pm 24.53$, versus $16.04 \pm 21.42$; p-value $<0.05$ ), also at the same time in placebo group MAP elevation was significantly more than paracetamol group ( $4.75 \pm 10.65$, versus $1.26 \pm 8.39$; p-value $<0.05)$.at this time RPP elevation in placebo group significantly was more than paracetamol group ( $3.22 \pm 4.53$, versus $1.54 \pm 3.61 \mathrm{p}$-value $<0.05)$ After 5 minute of intubation increasing of heart rate and mean arterial pressure was similar in both groups $(12.65 \pm 18.33$, versus $11.42 \pm 18.46 ; \mathrm{p}=0.45)(1.06 \pm 6.32$ versus $1.12 \pm 7.6 ; \mathrm{p}=0.69)$ and $\mathrm{RPP}$ reduction was similar in both groups $(1.54 \pm 3.98$ versus $1.12 \pm 3.43$ p-value<0.05)

Conclusion: Intravenous paracetamol as a premedication could reduces early hemodynamic changes after tracheal intubation in pediatrics with heart diseases.

\section{Epidemiology of myocardial contusion due to blunt chest trauma in pediatrics}

\author{
Ahmadipour M. ${ }^{1}$, Ahmadinejad M. ${ }^{1}$ \\ ${ }^{1}$ kerman university of medical sciences Iran, kerman
}

\section{Abstract}

Backgrounds: Blunt chest trauma is the most common cause of myocardial contusion in children but the absence of clear diagnostic criteria and reliable diagnostic tests makes reporting difficult so that there is not any exact statics about the incidence of cardiac injuries after blunt chest trauma specially in children. Material and method: In this descriptive, prospective study, from March 2014 to march 2017 all pediatrics who admitted to trauma center of Kerman province, evaluated for blunt chest trauma. All patients who had sign and symptom of myocardial contusion were consulted with pediatric cardiologist. of clear diagnostic criteria and reliable diagnostic tests makes reporting difficult so that there is not any exact statics about the incidence of cardiac injuries after blunt chest trauma specially in children. Result: During our study (3years) 168 traumatic children patients were admitted in trauma center. 31(18.4\%) patients had 
blunt chest trauma which $6(3.5 \%)$ of patients, had sign of myocardial contusion. $4(2.3 \%)$ person had sustained sinus tachycardia, $1(0.59 \%)$ of theme had left bundle branch block (LBBB) and $1(0.59 \%)$ patient had ST-depression.

All patients with myocardial contusion were transferred to ICU and echocardiography was performed for them by pediatric cardiologist. Two patients needed to vasopressors due to hypotension and low cardiac out put state.

All patients were discharged from ICU with a satisfactory general condition.

Trauma mechanism of $142(84.5 \%)$ patients, was road traffic accidents [ (28(16.6\%) were motorcyclist, 101(60.11\%) were car riders and13(7.73\%) were pedestrians ].

$21(12.5 \%)$ patients had falling down and 5(2.97\%) person had a history of falling a heavy body to their chest.

Conclusion: According to results of present study the most common cause of myocardial contusion in children is road traffic accidents(specially car accident), and sinus tachycardia is most common sign of myocardial contusion and seems that outcome of these patients is good.

\section{- The introduction of four cases of severe infantile dilated cardiomyopathy that were improved with medium-chain triglyceride}

\author{
Nobakht M. ${ }^{1}$ \\ ${ }^{1}$ Qazvin University of Medical Sciences Iran, Qazvin
}

\begin{abstract}
Backgrounds: Introduction: Infantile dilated cardiomyopathy is one of the most common myocardial diseases. Viral myocarditis, autoimmunity, and underlying genetic predisposition accepted as the main causes.

Material and method : Objective: The purpose of the study is to explain the role of low-fat diet in the development and treatment of infantile dilated cardiomyopathy.

Result: Methods and results: Four infants attended at different times with symptoms of heart failure. The final diagnosis in all of them was severe dilated cardiomyopathy. Their age was about four months and all of them were breastfed. Mothers of all infants were on a slimming diet due to their body fitness. The usual treatment started for all of them. In the first infant, the medium-chain triglyceride gave for better growth. The result was excellent. The levels of triglyceride were low in the three subsequent infant (mean: $50 \mathrm{mg} / \mathrm{dL}$ ). Due to the dramatic response, medium chain triglyceride was added to the treatment of other three infants. All of them recovered until two years old. Conclusion: Conclusion: Deprivation of adequate fat in lactating mothers, can lead to reduced breast milk fat and consequent low triglyceride level in the infant. Fat is the main fuel of the heart in infants after the neonatal period. Therefore, I recommend, level of serum triglyceride checked in any infant with dilated cardiomyopathy. Also, I recommend that mothers get adequate fat during lactation.
\end{abstract}

\section{Risk Factors of Congenital Heart Diseases A Case-Control Study}

\author{
Ahmadi A. ${ }^{1}$, Gharipour M. ${ }^{2}$, Navabi Z. S. ${ }^{3}$, \\ Heydari H. ${ }^{4}$
}

${ }^{1}$ Associate Professor Pediatric Cardiovascular Research Center Cardiovascular Research Institute Isfahan University of Medical Sciences Iran, Isfahan

${ }^{2}$ PhD Head Division of Genetic Studies Cardiovascular Research Institute Isfahan University of Medical Sciences Iran, Isfahan

${ }^{3}$ MSc in Health Education Promotion Pediatric Cardiovascular Research Center Cardiovascular Research Institute Isfahan University of Medical Sciences Iran, Isfahan

${ }^{4}$ MD Isfahan Cardiovascular Research Center Cardiovascular Research Institute Isfahan University of Medical Sciences Iran, Isfahan

\section{Abstract}

Backgrounds: Enhancing knowledge towards risk factors for Congenital Heart Disease (CHD) is important because of its high mortality and morbidity. Therefore, the aim of this study was to assess the risk factors of CHD.

Material and method: The participants of this case-control study were 474 consecutive children with congenital heart defects, and 436 sex and age-matched children without any evidences of congenital heart anomalies as well as their mothers whose information has been collected in Pediatric Cardiology Clinic of Noor Hospital, Isfahan, Iran. The study data was collected through checklists completed by the information of hospital records and interview with mothers of children with congenital heart defects as the case group, and healthy children as the control group.

Result: Based on the results of data analyses between case and control groups, obesity in mother, history of abortion, consanguineous marriage, family history of CHD, mother's disease and exposure with cigarette smoke and teratogens during pregnancy have significant relationship with incidence of congenital heart disease $(\mathrm{P}<0.05)$. Also, according results to the Multi-Variable Logistic Regression Modeling, obesity in mother $(\mathrm{OR}=1.57$, 95\% CI: 1.12-2.20), history of abortion ( $\mathrm{OR}=2.26,95 \% \mathrm{CI}$ : 1.38-3.74), consanguineous marriage ( $\mathrm{OR}=2.05,95 \% \mathrm{CI}: 1.48-2.83$ ), drug use during pregnancy ( $\mathrm{OR}=1.94,95 \% \mathrm{CI}: 1.30-2.89$ ), passive cigarette smoke exposure ( $\mathrm{OR}=2.07,95 \% \mathrm{CI}: 1.42-3.25)$ and teratogens $(\mathrm{OR}=2.36,95 \% \mathrm{CI}: 1.72-3.25)$ during pregnancy were significantly related to an increased risk of congenital heart disease.

Conclusion: Based on the results this study, prevention of mother's obesity before Pregnancy, improving Pregnancy health care, lowing consanguineous marriage, prompt treatment of mothers' disease, reducing exposure with cigarette smoke exposure and teratogens, may lower the occurrence of congenital heart disease.

\section{The commencement of congenital heart disease registry in Iran-Isfahan Methodology and Design}

\section{Dehghan B. ${ }^{1}$, Sabri M. R. ${ }^{1}$, Hosseinzadeh M. ${ }^{2}$, Ahmadi A. ${ }^{1}$, Ghaderian M. ${ }^{1}$, Sarrafzadegan N. ${ }^{1}$, Rouhafza H.}

${ }^{1}$ Isfahan University of medical sciences Iran, Isfahan

${ }^{2}$ Ahvaz University of medical sciences Iran, Ahvaz

\section{Abstract}

Backgrounds: Reported prevalence of CHDs (Congenital Heart Diseases) varies widely among studies worldwide. The incidence of CHD, total number of pediatric and adult Grown Up Congenital Heart disease (GUCH) is not determined in Iran. So, we have designed a system to register the information of patients with CHD for the first time in our country.

Material and method: CHD registry is a database in which the patients' data from hospitals or outpatient clinics are collected. We 
enrolled referrals from either new patients had come for evaluation of potential CHD or those who are being followed within the out patients clinics and entered their whole information in a website specifically designed for. All the information were collected from questionnaires by pediatric cardiologists as principle investigators. Result: From Apr 2017 to Nov 2018, after developing the forms and website, the first 558 files were evaluated by the quality control committee. 73 files (\%13) needed major revisions. Among them, 34 (\%46) files were omitted totally and the 39 remaining files were revised and completed. After that revision we have changed our questionnaire and gathered 1200 patients accordingly.

Conclusion: Registry of CHDs not only improves epidemiologic studies but also assists researchers to understand how much a disease management is useful and how to raise the quality of cares and outcomes. Moreover, this provides a better insight for policy makers to understand the extent of health-related problems as well as the issues related to the prevention and managements of congenital heart diseases all around the world.

\section{Oxygen therapy in patients with acute myocardial infraction the problem that is not yet solved}

Poorahmadieh F.

\begin{abstract}
Backgrounds: oxygen therapy in acute myocardial infraction is not as simple as saying a "yes" or a "no", on the contrary there are a number of unsolvable mysteries that need to be solved and therefore this study reviews the effects of oxygen therapy on patients with acute myocardial infraction based on scientific facts. Material and method: in this research, clinical practices from 2010 to 2018 from PubMed, Scoups, GoogleScholar databases were studied. The searched keywords consisted of oxygen inhalation, patients, myocardial infraction \& oxygen delivery.

Result: the number of clinical studies found was 6 . In these studies two groups were reviewed. Intervention oxygen therapy group and the room oxygen level control group. The studied results included Creatinine Phosphokinase enzyme, troponin enzyme, Arrhythmia, reoccurrence of myocardial infraction, size of the infraction, hospitalization, angina and the amount of drug use. In three studies results showed increase of myocardial damage and infraction size and increase in troponin and creatinine phosphokinase levels after the oxygen therapy, while the other three showed no difference in enzyme levels, mortality, hospitalization, infraction size, angina, and drug use in the two groups.

Conclusion: the exact effect of oxygen therapy in acute myocardial infraction has not been identified correctly and the difference in results in this particular field can furthermore emphasize the supervising teams caution on prescribing oxygen as a vasoactive medicine for patients with myocardial infraction and doing more clinical studies will help on identifying further pros and cons of prescribing oxygen.
\end{abstract}

\section{A case series of pulmonary hypertension in children under six months old with Atrial Septal defect}

\author{
Mahdavi Ch. ${ }^{1}$, Sabri M. R. ${ }^{1}$, Bigdelian H. ${ }^{1}$

\section{Abstract}

Backgrounds: Atrial Septal defect (ASD) is one of the most common congenital heart defects with prevalence of $8 \%$ to $10 \%$ in children and incidence of 56 per 100000 live birth. It is categorized according to the defect site. The most common form is ASD secundum with the defect in the central part of the atrial septum. Although pulmonary hypertension(PHTN) is seen with ASD in adulthood it's association with pulmonary hypertension in childhood is so rare. Material and method: In our case series study, we evaluate three children aged under six months old with ASD2 and PHTN. The patients were referred to clinic due to repeated pneumonia and FTT. They had fixed splitting of S2, loud P2 with hyper active pericardium in their physical exam. Their ECG revealed normal sinus rhythm, right axis deviation and rsR' in V1. Their echocardiography data showed right atrial and ventricular enlargement, moderate to large ASD2, moderate TR and PHTN without any additional defect. According to their findings all the patients were referred for surgical ASD closure.

Result: The patients were evaluated periodically after surgery. Their PHTN was improved and right atrium and ventricle size was normal during the follow up so that three months after the surgery all of them had normal pulmonary pressure and in one year normal size right atrium and ventricle.

Conclusion: Although ASD is rarely associated with PHTN in childhood, we should consider ASD closure in those children with PHTN in order to improve their PHTN.

\section{- Prevalence of Cardiac Involvement in Patients with Rheumatic Fever at Tabriz pediatric Hospital from 2006-2016}

\author{
Ghaffari Sh. ${ }^{1 *}$, Samady M. ${ }^{1}$, Jamei Khosroshahi \\ A. ${ }^{1}$, Molaiei A. ${ }^{1}$, Moradian M. ${ }^{2}$, Soleimany N. ${ }^{1}$
}

${ }^{1}$ Tabriz University of Medical Sciences Iran, Tabriz

${ }_{2}^{2}$ Iran University of Medical Sciences Iran, Tehran

\section{Abstract}

Backgrounds: The purpose of this study was to investigate the prevalence of cardiac involvement in the patients with acute rheumatic fever during the last 10 years.

Material and method : First, in coordination with the medical evidence unit, the list of patients admitted with RF is being prepared in pediatrics hospital for the last 10 years. We will take the information we need (including cardiac involvement of acute rheumatic fever and demographic information of patients) according to the check list that we prepared previously.

Result: In the study of 48 patients with acute rheumatic fever, who were hospitalized over the last 10 years, 35 patients (72.9\%) had cordites and 36 patients (73\%) had arthritis. The most common valvular involvement was mitral valve regurgitation (66.7\%) followed by aortic valve insufficiency (35.5\%) and mitral and aortic valves regurgitation simultaneously. Serum titer of anti-streptoly$\sin \mathrm{O}$ (ASO) during hospitalization and the medical treatment was slowly decreasing and the highest serum liter of that was while admission. There was no significant connection between carditis with probability of age, sex, body weight, rheumatic arthritis, socioeconomic level of family, valvular disease of ASO serum liter. Conclusion: The finding of this study showed that heart complications such as carditis with high incidence of over $70 \%$ were associated with acute RF disease. Noting that Middle East is among 
the high prevalence and incidence of acute RF and Iran is not an exception, so long term screening and follow up of carditis in acute RF patients is necessary.

\section{Evaluation of pulmonary complications after open cardiac surgery in children with Congenital anomalies}

Jamei Khosroshahi A. ${ }^{*}$, Gaffarei Sh. ${ }^{1}$, Samadi M. ${ }^{1}$, Molaei A. ${ }^{1}$, Moradyan P. ${ }^{2}$

${ }^{1}$ Shahid madani hospital Iran, Tabriz

${ }^{2}$ Shahid rajaei hospital Iran, Tehran

\section{Abstract}

Backgrounds: Cardiac surgery due to congenital heart disease is one of the mortality and morbidity causes in children. One of the most important complications of these surgeries is pulmonary disorders. Thus, the aim of this study is to evaluate the incidence of pulmonary complications following open cardiac surgeries in order to better evaluate the present status and the importance of paying attention to this issue for reducing mortality, admission period in PICU, and additional costs for health and hygiene organizations of the country

Material and method : The present study was a descriptivecross sectional study on the patients admitted after a definite diagnosis of the cardiac disorder in cardiovascular surgery service. The sampling method was simple sampling including data of the cases of the children suffering congenital heart disorder in age between 1 month up to 18 years old who were admitted since March 2015 until March 2016 and underwent cardiac surgery Result: Among 296 cases, $54.7 \%$ were male and $45.3 \%$ were female. Mean age was two years and 9 months and mean weight was 11.5 $( \pm 6.7) \mathrm{Kg}$. Pneumonia was seen in 15 patients (5.1\%) and pleural effusion was seen in 22.7 percent. Pulmonary complications were less in older ages (correlation coefficient $=-0.123, \mathrm{P}=0.01$ ). Also, in patients staying more days in PICU, significantly more complications were seen (Correlation coefficient $=0.33, \mathrm{P}=0.0001$ ). The most prevalent complications were seen in the surgery of cyanotic disorders without shunt application (37.9\%) and the least complications were seen in the surgery of valvulopathies (16\%).

Conclusion: The result of the present study demonstrates that in older ages the pulmonary complications were less. Also in patients staying more days in PICU complications were higher. Thus, the pulmonary complications can be considered as one of the causes of longer staying of patients in PICU and consequently higher financial burdens of patients. The most prevalent complications were due to surgery of cyanotic disorders without shunt application and the least complications were seen in the surgery of valvulopathies

\section{The Frequency rate of early} postoperative complete heart block in pediatric patients submitted to openheart surgery for congenital heart disease

Jamei Khosroshahi A. ${ }^{1 *}$, Samadi M. ${ }^{1}$, Molaei A. ${ }^{1}$, Gaffarei Sh. ${ }^{1}$

\section{Abstract}

Backgrounds: Degeneration of cardiac conduction system, acute myocardial infarction, congenital disorders and cardiac abnormalities are the most common causes of complete heart block. Complete heart block following surgical repair of congenital heart disease is important and it is one of the main causes of death and cardiovascular failure following surgical repair of congenital heart disorders. Using a pacemaker is the choice for treatment of complete heart block. The aim of this study was to determine the frequency of early postoperative complete heart block in patients with congenital heart disease and to determine the need for a temporary and permanent pacemaker.

Material and method : This is a cross-sectional descriptiveanalytic study in which children with congenital heart defects who had undergone open heart surgery in the Shahid Madani Hospital of Tabriz from the beginning of the year 1390 to the end of the year 1396 were studied. Patients with advanced early postoperative complete heart block were analyzed. We identified the cases that recovered spontaneously and the group that needed temporary pacemaker (TPM) or permanent pacemakers (PPM). Finally, the frequency of the complete heart block and the need for a temporary pacemaker and permanent pacemaker were determined.

Result: Out of 2100 patients, 109 cases suffered from early postoperative complete heart block. The Frequency of early postoperative complete heart block in congenital heart disease was $5.19 \%$. Out Of 109 patients, 69 patients (63.3\% of patients with early complete heart block and $28.3 \%$ of all patients) required pacemakers, all of them received temporary pacemakers, and 9 of them (8.3\% Patients with early complete heart block and $0.42 \%$ of all patients) needed permanent pacemakers. The rest of the patients recovered without a pacemaker or died before using the pacemaker.

Conclusion: In this study, Frequency of early postoperative complete heart block among patients with congenital heart disease was 5.19\%. The need for pacemaker was high and most of the patients had recovery in heart rhythm without having a permanent pacemaker, with or without a temporary pacemaker.

\section{Prevalence of congenital heart disease in pregestational and gestationaldiabetes melitus}

\author{
Soltani M. ${ }^{1 *}$ \\ Sarem hospital Iran, Tehran
}

\begin{abstract}
Backgrounds: The role of maternal pregestational diabetes melitus(PGDM) in occurrence of fetal congenital heart disease(CHD)is completely well known but the risk of CHD in fetuses of maternal gestational diabetes melitus( GDM) is less recognized.

Material and method: The aim of this observational study was to compare the occurrence of congenital heart disease in these two referral groups between 2014 to 2017 and to evaluate the importance of fetal echocardiography in women with GDM.

Result: During the study period 96 women with PGDM were referred for fetal echocardiography and a fetal congenital heart defect was diagnosed in 3 cases (3.1\%). In the GDM 112 women
\end{abstract}


were referred for screening and 3 cases (3.7\%) of fetal CHD were detected.

Conclusion: Accordings to our data in this study, women with GDM to be at an increased risk of having a baby with CHD. This risk might be due to a combination of hyperglycaemia, insulin resistance, an elevated BMI and more importantly, undiagnosed pre-gestational diabetes. At present, we accept that detailed fetal echocardiogrphy in mothers with GDM is necessary Until more data from larger studies obtain.

Keywords: Congenital heart disease; Pregestational diabetes melitus; Gestational diabetes mellitus

\section{Determining the prevalence} of early and late complications following the Truncus Arteriosus reconstructive surgery at Shahid Rajaie Cardiovascular Center between 2006 and 2015

\author{
Mortaz Hejri G. ${ }^{*}$, Tabib A. ${ }^{2}$, Mahdavi M. ${ }^{1}$, \\ Shahmohammadi A. A. ${ }^{1}$
}

${ }^{1}$ Rajaie Cardiovascular Medical and Research CenterIran University of Medical SciencesTehranIran Iran, Tehran

${ }^{2}$ Heart Valve Disease Research Center Rajaei Cardiovascular Medical and Research Center Iran University of Medical Science Tehran Iran Iran, tehran

\footnotetext{
Abstract

Backgrounds: Introduction:Truncus arteriosus (TA) is a rare congenital cardiovascular disease. Since the very first repair of truncus arteriosus more than 30 years ago, major advances have been made in the management of this lesion, transforming it from a fatal condition early in life in over $50 \%$ of patients, to one in which neonatal repair is increasingly common and increasingly successful. In this study we determined the short- and long-term complications of TA repair in patients undergoing surgical treatment at Shahid Rajaie Cardiovascular, Medical and Research Center.

Material and method: This case series study was conducted to determine the prevalence of early and late complications following truncus arteriosus repair in the period 2006 to 2015 Demographic data,early post op outcomes (early mortality at operating room,mortality in ICU), other complications occurred in ICU and long-term outcomes (delayed surgical outcomes including mortality, delayed complications, need for reoperation, and long-term survival rates) were records from all patients.

Results: In this study, the medical records of 62 children aged 1 to 36 months were examined. With a mean age of 5.8 months, $54.84 \%$ of the subjects were boys and $45.16 \%$ were girls. Truncus type, the most prevalent cases were type I and type II (83.87\% and $16.12 \%$, respectively). The most and least common types of surgery used in this study were aortic homograft (29.03\%) and Gore-Tex tube (6.45\%).Hospital mortality was $48 \%$.Need for reoperation was $22 \%$ and moderate to severe aortic regurgitation was $25 \%$.

Conclusion: Based on our experience in this study our long term outcomes similar with other centers .But

Our mortality in early post operation in lower weight and age was more than other centers. It seems that there is still insufficient ability to perform restoration of Truncus arteriosus at an earlier age.
}

Right atrium thrombosis as a late onset complication of surgical ASD closure in a 6-year-old girl

\author{
Zolfigol A. ${ }^{{ }^{*}}$, Radvar M. ${ }^{1}$
}

${ }^{1}$ UMSU Iran, Urmia

\begin{abstract}
Backgrounds: Thrombus formation in right atrium is a rare complication of surgical ASD closure and has potential of causing pulmonary embo

Material and method: Case report

Result: A 6-yr old girl admitted to our hospital, because routine transthoracic echocardiography(TTE) revealed a linear thrombus $\left(8^{*} 20 \mathrm{~mm}\right)$ at the junction of SVC to RA with no clinical symptoms. The patient had history of surgical closure of large ASD secundum type in 11 months ago. The cardiac magnetic resonance imaging(MRI) confirmed echocardiographic findings. Heparin drip at usual infusion dose and Aspirin(ASA) with antithrombotic dose were started. On the 5th day of treatment, no thrombus was detected in TTE and heart MRI. During of treatment the patient had not any symptoms and signs of pulmonary embolism. On the 9th day of hospitalization, the patient was discharged with a good general condition. In follow up evaluation 6 months' later the patient had not any evidence for clot formation in RA.

Conclusion: RA thrombosis could be a late onset complication of surgical ASD closure and anticoagulation therapy with heparin and ASA are an effective and safe treatment

\section{Diagnostic and therapeutic pediatric} cardiac catheterization A large singlecenter experience
\end{abstract}

\author{
Jafari F. ${ }^{*}$, Tabib A. ${ }^{1}$, Mortezaeian H. ${ }^{1}$, Mahdavi \\ M. ${ }^{1}$, Vesal A. ${ }^{1}$, Meraji M. ${ }^{1}$ \\ Rajaie Cardiovascular Medical and Research Center Iran, tehran
}

\begin{abstract}
Backgrounds: This study identifies characteristics of children goes under cardiac catheterization and evaluate various issues related to diagnostic and therapeutic pediatric cardiac catheterization. Material and method: This is a prospective case series study. A total of 576 Children $(<15 \mathrm{y} / 0)$ with congenital heart disease who received cardiac catheterization in Rajaie Cardiovascular, Medical \& Research Center in Tehran from November 2015 to February 2016 were studied. Patient's characteristics and catheterization information were collected with a data collection form. The SPSS 22.0 software was used for statistical analysis.

Result: Total of 580 patients were entered to the cath lab, 576 of which performed the cardiac catheterization procedure. The most common age group was children aged 1 month to 5 years (66\%). The lowest patient weight was $2.2 \mathrm{~kg}$. $54.6 \%$ of the catheterizations were diagnostic and $45.4 \%$ were therapeutic (interventional). In the age group under one month and over ten years, interventional catheterization was more than diagnostic catheterization. According to procedure-type risk categories, 86.1\% of procedures were classified as category $1 \& 2$ (lower risk) and
\end{abstract}


$13.9 \%$ as category $3 \& 4$ (higher risk). Major and moderate complications occurred in $2.5 \%$ and $14.2 \%$ of cases respectively.

Conclusion: Diagnostic catheterization still plays an important role in assessing anatomic and hemodynamic status, especially in complex congenital heart diseases. Therapeutic catheterizations increasingly used as a suitable and accepted therapy for many congenital heart defects.

\section{- Sever aortic stenosis A case report of child neglect}

\author{
Jafari F. ${ }^{{ }^{*}}$, Mahdavi M. ${ }^{1}$, Gholampour M. ${ }^{1}$ \\ ${ }^{1}$ Rajaie Cardiovascular Medical and Research Center Iran, Tehran
}

\section{Abstract}

Backgrounds: Aortic stenosis is a progressive disease that early diagnosis and treatment can reduce morbidity and mortality. Child neglect is a deficit in meeting a child's basic needs, for example the failure to provide adequate health care, as well as their physical, emotional, and social needs.

Material and method : 11-year-old girl presented to the emergency room with signs and symptoms of heart failure. In echocardiography sever aortic stenosis (AS), patent ductus arteriosus (PDA) and left ventricular ejection fraction (LVEF) between 10-15\% was detected. According to the mother, parents have been aware of their child's heart disease since neonatal period, but they did not follow their child's disease due to fear of medical intervention and hope for spontaneous recovery. Although the child had progressive dyspnea and edema from 4 weeks earlier, the parents did not take the child to the physicians. The patient referred to tertiary center and MRI reported LVEF and RVEF $13 \%$ and $26 \%$ respectively. Result: After 20 days of medical management, the patient had surgery (aortic valve repair, commissurotomy, and myectomy and PDA closure). Echocardiography estimated the LVEF about 30 $\%$ and $50 \%, 2$ months and 10 months after the surgery respectively. Conclusion: It seems false beliefs and lack of knowledge of parents are the cause of child neglect in our case. Although cardiac function returns to a better level after surgery, it is obvious that Child neglect has had a negative effect on the child's performance and quality of life.

Keywords: aortic stenosis, child neglect

\section{- Vitamin E deficiency as a risk factor for cardiovascular diseases}

\section{Aghaei M. *}

\begin{abstract}
Backgrounds: Cardiovascular diseases are one of the most common health-related problems and the number of the patients with cardiovascular diseases increases each year.

Material and method: This review study has been conducted by searching the related works in the databases of Google Scholar, Pubmed, PMC, Springer, and Elsevier, published from 2014 to 2018. Result: $\alpha$-Tocopherol is the most common form of vitamin $E$ in human tissues. Increased oxidative stress is an important mediator of endothelial injury in hypertension pathology, associated with increased oxidant protein production, such as hydrogen peroxide superoxidation, decreased nitric oxide synthesis, and decreased
\end{abstract}

biological capacity of antioxidants. Oxidative stress may lead to endothelial dysfunction, inflammation, hypertrophy, fibrosis, and angiogenesis associated with hypertensive vascular variations. Vitamin E deficiency can provide a constraint for micro ribonucleic acids (miRNAs) networks, and this may lead to the progression of atherosclerosis. Vitamin E deficiency causes adverse effects in cardiovascular systems. However, the effect of its use in the treatment of diseases is still not fully understood.

Conclusion: Considering the above results, a good amount of this substance should be provided and it should be replaced before the complications occur.

\section{Study of Correlation between Vitamin D and Coronary Artery Calcification}

\author{
Sajjadieh Khajouei A. ${ }^{{ }^{*}}$, Sajjadieh Khajouei H. ${ }^{1}$, \\ Kasaii Z. ${ }^{1}$
}

${ }^{1}$ Isfahan University of medical sciences Iran, Isfahan

\section{Abstract}

Backgrounds: considering the role of vitamin D in cardiovascular disease (CVD) and the relationship between coronary artery calcium score (CAC) and CVD, we aimed to investigate the association between serum level of vitamin D level and CAC.

Material and method: in this cross sectional study, 83 consecutive patients were referred for performing CT angiography to measure CAC. An educated nurse filled a questionnaire containing the information about past medical history of diabetes mellitus, hypertension, hyperlipidemia and smoking for each patient. Serum samples were collected for the evaluation of vitamin D level. After adjustment with confounders, we extracted and analyzed the data to assess relationship between vitamin D level and CAC. Result: this study included the final number of 67 patients of which 37 had positive CAC (mean CAC: 128.1) and patients had 30 negative CAC (mean CAC: 0 ). There was no significant association between CAC and vitamin D levels ((Pearson coefficient= -0.01 and Spearman coefficient $=-0.03, P$ value $>0.05)$ but the past history of hyperlipidemia and D.M were more prevalent in CACpatients than those without CAC $(62.2 \%$ and $32.4 \%$ vs. $60 \%$ and 13.3\%) (Pvalue $<0.05)$.

Conclusion: our study revealed that vitamin D had no significant relationship with CAC. Furthermore, among major risk factors of cardiovascular diseases, diabetes mellitus and hyperlipidemia are associated with higher CAC scores

\section{The relationship between $Q T$ interval and Severe pulmonary hypertension in patients with congenital heart disease}

\author{
Tanasan A. ${ }^{1 *}$, Moradi m. ${ }^{2}$, Esna Ashari F. ${ }^{3}$, \\ Hasani Z. ${ }^{4}$
}

${ }^{1}$ Assistant professor of Pediatrics Cardiology, Department Of Pediatrics, Besat Hospital, Hamadan University of Medical Sciences, Hamadan, Iran.

${ }^{2}$ Assistant professor of Cardiology, Farshchian Heart Center, Hamadan University of Medical Science, Hamadan, Iran.

${ }^{3}$ Associate Professor of community medicine, Department of community medicine, Hamadan University of Medical Sciences, Hamadan, Iran.

${ }^{4}$ Medical student, Department Of Pediatrics, Hamadan University of Medical Science, Hamadan, Iran 


\section{Abstract}

Backgrounds: Introduction: Patients with severe pulmonary hypertension due to severe right ventricular dysfunction are at increased risk of severe arrhythmia. In some studies, an increase in QT interval, which could be the trigger of these arrhythmia, were reported in these patients.

Material and method: In this study, we classified 40 patients with $\mathrm{PH}$ and congenital heart disease, which were diagnosed with echocardiography and catheterization, in two groups of mild PH and moderate to severe PH. Then we measured the QRS, QTc interval with the ECG and assessed the right ventricular function with RVMPI by echocardiography.

Result: Of 40 patients with $\mathrm{PH}, 19$ subjects were in the mild $\mathrm{PH}$ group and 21 subjects were in Moderate to severe PH group, in which 9 patients had irreversible PH or Eisenmenger syndrome. QTC in mild PH group and severe PH group were 434.5 \pm 20.2 , $450.8 \pm 22.6$ respectively that was not significant $(\mathrm{p}=/ 105)$ but $\mathrm{QRS}$ time significantly prolonged in the severe $\mathrm{PH}$ group compared to mild $\mathrm{PH}$ group $94.7 \pm 15.4 \mathrm{~ms}$ versus $109 \pm 22.1 \mathrm{~ms}$ ( $\mathrm{p}=0.009$ ). In the severe PH group in patients with Eisenmenger syndrome compared to non Eisenmenger patients , QTC ((9):461.4 $\pm 104 \mathrm{~ms}$ vs (12):442.9 $\pm 26.3 \mathrm{~ms}(\mathrm{p}=0.042)$ and $\mathrm{QRS}$ time ((9):116.6 $\pm 23.6 \mathrm{~ms}$ vs (12):104.5 $\pm 20 \mathrm{~ms}(\mathrm{p}=0.002)$, were significantly different. RV MPI in control group and non Eisenmenger patients and Eisenmenger patients were $0.36,0.38,0.5$ respectively

Conclusion: In patients with Eisenmenger syndrome, an increase in QTC interval might be correlated independently with the RV function.

\section{Pulmonary sling in 16 month old boy case report}

Khalilian M. R. ${ }^{1 *}$, Baghaei Tehrani R. ${ }^{1}$, Dabgagh A. ${ }^{1}$, Ansari Aval Z. ${ }^{1}$

${ }^{1}$ Shahid beheshti university of medical sciensec Iran, tehran

\section{Abstract}

Backgrounds: Case report: A 16 month old boy was referred to the outpatient clinic. He was a full term baby. He had been noted to be breathless and wheezy from birth, with a cough that was occasionally productive. He had history of hospitalization three times for respiratory problems. On examination he appeared a healthy child, no cyanosis and with no clubbing; his height was on the 25th percentile and his weight on the 50th percentile. He had both inspiratory and expiratory rhonchi. Heart sounds was holosystolic murmur due to ventricular septal defect.

Material and method: A chest radiograph showed mild increased cardiothoracic ratio and normal pulmonary blood flow (fig 1). Echocardiography showed that small Perimembranous Ventricular Septal Defect and aberrant left pulmonary artery arising from the right pulmonary artery (fig 2).

Result: CT angiography showed pulmonary sling (fig 3). Bronchoscopy showed multiple complete rings from thoracic inlet to $1 \mathrm{~cm}$ above carina narrowing (approximately 8 cartilage) with significant tracheal narrowing at 3 last complete rings (> 50\% obstruction, Grade 2) (fig 4).surgery had performed (fig 5). After surgical procedure he had a better condition.

Conclusion: Discussion: Infants with pulmonary artery sling usually present in the first year of life. The anomaly, if not corrected, can be fatal within the first year of life. Patients with pulmonary artery sling present with respiratory symptoms. Other symptoms and signs include cyanosis, stridor, inspiratory and expiratory rhonchi, apneic episodes, asymmetry of the chest, and difficulty in feeding. Pulmonary sling is an anomalous course of the left pulmonary artery, which arises from the proximal right pulmonary artery and runs posterior to the right main bronchus and trachea before entering the hilum of the left lung. Complete tracheal cartilage rings causing longsegment stenosis are commonly associated with pulmonary artery slings. This anomaly is frequently associated with congenital heart defect (septal defects, patent ductus arteriosus, atrioventricular septal defects, and aortic arch anomalies) in $30 \%$ to $40 \%$ of cases. 



\section{A}

Abbasi Dolatabadi Z.

Abdi Oskouei M.

Abdoos M.

Abrahimi A.

Abtahi F

Afshin Nia N.

Aghaamoo Sh.

Aghaei M.

Ahmadi A.

Ahmadi F.

Ahmadinejad M.

Ahmadipour M.

Ahmadi S.

Ahmadi Z.

Ajami G.

Akbari M.

Akbarzadeh M.

Akhavan Z.

Alavi S.

$\mathrm{Al}$ E Mohamad M.

Alemohammad M.

Alemzadeh Ansari M.

Alikhani Boroujeni R.

Ali Rezaei O.

Alizadeh A.

Alizadehasl A.

Alizadeh Ghavidel A.

Alkenzawi $\mathrm{H}$.

Almandil A.

Amanollahi F.

Amin A.

Aminbeidokhty A.

Amirghofran A.

Amoozgar $\mathrm{H}$.

Amouzegar Zavareh S.

Amrollahi S.

Anbiaie R.

Ansari Aval Z.
42, 44, 59 46 58 58 49

9

35,42 35

15

11,18

$7,37,38$

6, 10, 36, 40

34

54

55

56

57

$7,8,27,35$

6

9

32

$5,14,27,50$

43

15

13

11

44

57

64

Arabloo M.

Arzani P.

24

Asadzadeh $\mathrm{H}$. $\quad 16$

Asad Zade L. $\quad 12$

Asgari P. $\quad 52$

Atrkarroushan Z. 28

Attarbashi Moghadam B. $\quad 23$

Austine N. $\quad 35$

Azadeh P. $\quad 57$

Azarfarin R. $\quad 9,21,40$

Azdaki N. 33

Azimi M. $\quad 49$

Azizi B. $\quad 19,48$

Azizi Z.

$>\mathbf{B}$

Babaei T.

11

Babapour S.

$45,52,54$

Baghaei Tehrani R. 64

Bagheri I. $\quad 16$

Bagherinasab M. 19

Baharestani B. $\quad 7$

Bahramnezhad F. $\quad 52$

Bahremand M. $\quad 38$

Bakhshaei M. $\quad 10$

Bakhshandeh A. 6, 7, 10, 36, 37, 38, 40

Bakhshandeh $\mathrm{H}$. $\quad 5,11,27,35,56$

Bakhtiari A. $\quad 7,37$

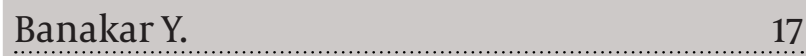

Banazade A. $\quad 58$

Barekatain B. $\quad 58$

Barzabadi Farahani Z. $\quad 54$

Bayanati M. 43

Bayati P. $\quad 39$

Bayat R. $\quad 20$

Behzadnia N. $\quad 9$

Beikverdi M. 46

Berahman M.

$66 \quad$ Iran J Psychiatry Behav Sci 


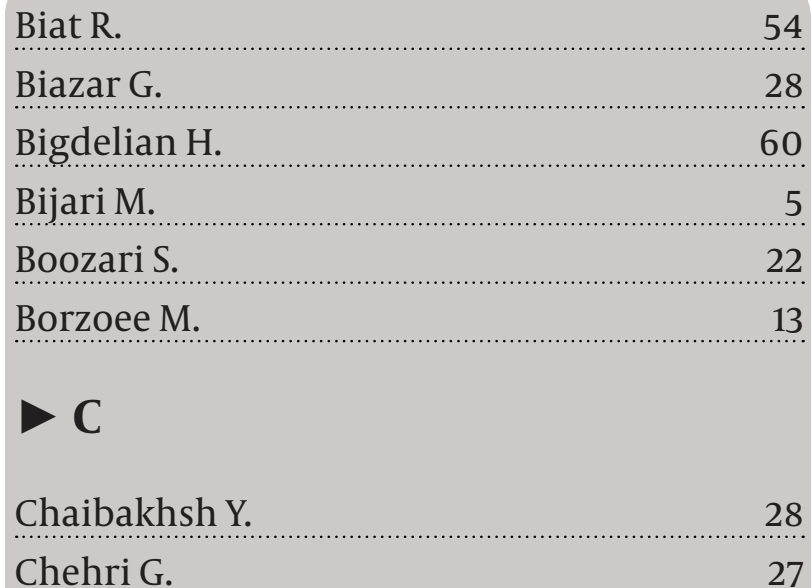

\section{D}

Dabbaghipour N.

Dabgagh A.

Dabirian A.

Dadkhah M.

Daliri M.

Dehestani A.

Dehghan B

Dehghan B.

Dehghani A.

Dehghani E.

Dehghani H.

Dehghani K.

Dianatkhah M.

\section{E}

Eazti M.

Edraki M.

Eghbalibabadi M.

Emami F.

Emkanjoo Z.

Emrahi L.

Ershad S.

Eskandari S.

Esmaeili R.

Esna Ashari F.

Esteki T.
23, 24

64

54

15

8 44

12

59

35

27

47

47

5,28

32,34

32

27,56

\section{3}

\section{$\mathbf{F}$}

Faghihi T. 44

Farasatkish R.

9,40

Faritous Z.

9,40

Farjam Fazelifar A. $\quad 56$

Farzaneghan B.

Fasihnia S. $\quad 25$

Fatahi M. $\quad 18$

Fatemian $\mathrm{H}$. $\quad 42$

Fazelifar A. F. $\quad 26$

Feizi A. 32

Firouzi A. $\quad 8,42,43$

Foroutan M. 18

Foudazi H.

Fouladi Vanda $\mathrm{H}$.

$\mathbf{G}$

Gaffarei Sh.

61

Garak Yaraghi M. $\quad 34$

Ghaderian M.

Ghadrdoost B.

$44,58,59$

Ghaffari S.

26, 57

14

60

Ghaffari Sh.

51

Ghanbari Afra L.

51

Ghanbari Afra M.

16,50

Ghanbari B.

26

Ghanbari M.

Gharipour M.

52

Ghasemian Khojasteh Z.

48

Gheysardoost M.

17

Ghodrati M.

29

Ghods K.

20

Gholampour Dehaki M.

7, 21, 63

Gholampour M.

42

Ghorbani B.

Golpira R.

56, 57

Golshahi J.

Gorjipour F. 


\section{$>\mathrm{H}$}

Hadavand N.

Hadipoorzadeh F.

Haghjoo M.

Halvaei M.

Hanifi Z.

Harati $\mathrm{H}$.

Hasani Z.

Hasanpour Dargah M.

Hashemifard $\mathrm{O}$.

Hassani E.

Hassanzadeh A.

Heidarali M.

Heidari Nia S.

Heidarpour M.

Hemati M.

Heshmatghahdarijani

Heshmat-Ghahdarijani K.

Hesmat K.

Heydaralizade R.

Heydari $\mathrm{H}$.

Hiradfar A.

Hoseini Marzango S.

Hosseini K.

Hosseini M.

Hosseini S.

Hosseinzadeh M.

Hutter $\mathrm{H}$.

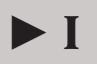

Imanipour M.

Imani S.

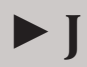

Jadbabaei A.

Jafari F.

Jafaripoor I.
Hamadani K.

Hasan Zade K.

27,56

20,54

50

44

63

42

12

35

41

43

56

19

32,34

55

5

28

4

42

59

43

52,54

32

4,50

$7,18,27$

20, 59

36

49

53

68

Iran J Psychiatry Behav Sci
Jahangirifard A.

Jahantigh Akbari N.

55

Jalali A.

21

Jalali F.

34

Jalaly P.

47

Jamali M.

48

Jamali Z.

40

Jamei Khosroshahi A.

Janjani P.

Jargouei Esparti M.

Javadinejad $\mathrm{M}$.

Javaherforoosh Zadeh F. $\quad 11,41$

Javaherian M.

24

Jenab Y.

12

Jozan M.

$>\mathrm{K}$

Kalani Z.

16

Karami A.

20,54

Karami N.

10,41

Karamnezhad M.

37

Kargar S.

42

Kasaii Z.

35,63

Kassani A.

7, 37, 38

Keshavarz K.

14

Khajali Z.

Khaki M.

27

Khaleghparast S.

39

Khalilian M.R.

16,50

Khezerloy Agdam N.

Khorshidi H.

64

Khosravi A.

$32,34,56$

Khosravian Arab T.

23

Khosravi Bizhaem S. $\quad 33$

Kojuri J.

42

Kundi M.

Lak M. 
M

Madadi Sh.

Mahdavi Ch.

Mahdavi M.

Mahdavi Shahri S.

Mahoori A.

Malekan Rad E.

Maleki A

Maleki M.

Malek Kandi M.

Malekzadeh M.

Mansouri R.

Marzban V.

Masoumi M.

Maziar Gholampour Dehaki M.

Mehdizadegan N.

Mehdizade $\mathrm{M}$

Mehrabanian M.

Mehrpooya M.

Meraji M.

Miraki S.

Mirmansouri A.

Mirmohammadsadeghi A.

Moarref A.

Moazenzadeh M.

Moezi S.

Mofid M.

Moftakharzadeh A.

Mohamadi F.

Mohammadi E.

Mohammadifard N.

Mohammadi $\mathrm{H}$.

Mohammadzadeh Jouryabi A.

Mohebbi A.

Mohebbi B.

Mohsenabadi M.

Mohsenipour Foumani A.

Moini M.

16,50

$16,27,50,57$
Mojtahedi M.

Mokhtaran M.

Molaei A.

13, 61

Molaie A.

14

Molaiei A.

60

Momeni M.

44

Moradian M.

Moradi m.

$14,27,39,60$

Moradyan P.

63

Mortazavi S.

61

Mortaz Hejri G.

Mortazian M.

62

18

Mortezaeian $\mathrm{H}$.

42,62

Mosallanezhad Z.

25

Moslem F.

Mousavi Shabestari M.

Mousavi Shabestary M.

52

Mozayanimonfared A.

$>\mathbf{N}$

Nabhani F.

Naderi N.

$14,27,50$

Naghashzadeh F.

Naghshtabrizi B.

Najafi $\mathrm{H}$.

Nami M.

45

Naseri H.

Nasrizadeh Moghaddam S.

33,48

Nassiri Sheikhani N.

28

Navabi Shirazi M.

Navabi Z.S.

Nazari M.

Nezafati M.

Nezafati P.

Niknam S.

Nikoo M.

Nikpajooh A.

Niroomand $M$.

Nobakht M. 
Noohi F.

Norouzi Z.

Nouri F.

$>0$

Omrani Gh.

Oraki F.

Ordibehesht N.

Oveisi M.

\section{$>\mathbf{P}$}

Pahlavan S.

Pakcheshm B.

Parchami F.

Parizad R.

Parsaee M.

Pashaie S.

Peighambari M. M.

Peiravian F.

Pishnamaz Ahari N.

Poorahmadieh F.

Pouraliakbar H.

Pourhoseingholi M.

Pur Shahbazi Liqvan E.

\section{$\mathbf{R}$}

Radvar M.

Rafiee S.

Rafi Khorgami M.

Rahimi F.

Rahimi H.

Rahmanian M.

Rahmani M.

Rahmati Yami M.

Rahsidi Ghader F.

Rajaei S.

Ranjbar M.

Rashid H.

Rashidinejad H.
27,57

34,43

56

27

22

51

50,57

4

16

51

52,53

27

50

27

15

53

60

39

45,54

53

62

21

27

24

18

$6,7,10,36,37,38,40$

42

26

27

15

34

13

32

Rastgar Koutenaei F.

Ravanbod R.

$22,26,28$

Rezaeian N.

39

Rezaei Y.

27

Rezaeiyan Y.

26

Rezvan M.

20

Roghanidehkordi F.

Roohafza $\mathrm{H}$.

$4,5,28$

Rouhafza H.

59

Rozbahani M.

$>\mathrm{S}$

Saadati B.

49

Saba M.

Saberi K.

$6,10,36,38,40,52$

Sabri M.

12

Sabri M. R.

59,60

Sadeghi A.

9, 40

Sadeghian M.

23

Sadeghifar M.

4

Sadeghi M.

$4,5,28,35$

Sadeghi S.

35

Sadeghi T.

18

Saedi S.

Saeidi M.

22,28

Safari Soltanabad A.

48

Safizadeh $\mathrm{H}$.

32

Sajjadieh Khajouei A.

35,63

Sajjadieh Khajouei H.

35,63

Salahi S.

Salehi M.

$6,7,10,36,37,38,40$

Salehi P.

34

Salehzade A.

21

Salesi M.

16

Salimbahrami S.

10

Salman Dehkordi F.

49

Salmanian S.

Salmani S.

Samadi M.

14,61 
INDEX

Samady M.

Tanasan A.

Samiei N.

Tarbiat M.

10

Saneipour Z.

Sarrafzadegan N.

$4,5,28,32,56,59$

Sattarzadeh R.

Tasmeh S.

21

Tavoosi A.

Sayadmansor M.

Tirgarfakheri K.

Sayadpour Zanjani K.

46

Sedighinejad A.

Torkan N.

Totonchi Z.

$18,21,35$

Totounchi Z.

Serdijn W. A.

Toubaee S.

Seylani K.

49, 51

Shabanian R.

Toufan Tabrizi M.

Shadmehr A.

Shafie D.

32,34

Shafieyoon S. 32

Shahbazi S.

Shahbazpour J.

Shahmohammadi A. A.

Sharifi M.

Sharif Kashani B.

$>$ V

Vafai $\mathrm{H}$.

Vahedparast H.

Vahidi $\mathrm{H}$.

Vakhshoori M.

$5,28,32,34$

Vakilian F.

Vesal A.

Shefaat S.

$\checkmark \mathrm{Y}$

Shirafkan R

Shirvani Y.

Shoaei O.

Yadegari M.

Yadegary M.

Shojaei Fard M.

Yaghoubi A.

Shomali A.

Yazdi A.

Skeikhi B.

Yeganeh S.

Sobhanian K.

Soleimany N.

Soltani M.

\section{$\checkmark \mathrm{Z}$}

Zafarnejad $\mathrm{M}$.

Sultan S.

Zakerimoghadam M.

49, 51

\section{$T$}

Zamani M.

Zamirian M.

Zamiri B.

Taban Sadeghi M.

Tabib A.

27, 62

Taghavi S.

$14,21,27,50$

Taghizadeh A.

Zareh F.

Zarei Mahmoodabadi A.

46

Zarrabi K.

Tahmasvand $\mathrm{M}$.

Zebardast J.

Taiyari S.

Ziyaeifard M.

$9,11,18,40$

Tamimi T.

Ziya Sarabi P.

Zolfigol A. 
